\title{
THE FREE QUASIWORLD. FREELY QUASICONFORMAL AND RELATED MAPS IN BANACH SPACES
}

\author{
JUSSIV V ÄISÄL $\ddot{A}$ \\ Matematiikan laitos, Helsingin yliopisto \\ PL 4 (Yliopistonkatu 5) \\ FIN-00014 Helsinki, Finland \\ E-mail: jvaisala@cc.helsinki.fi
}

\section{Contents}

1. Introduction 55

2. Uniform continuity and quasiconvexity 58

3. Quasihyperbolic metric 61

4. Maps in the free quasiworld 65

5. Quasihyperbolic maps 66

6. Quasisymmetric and quasimöbius maps 73

7. FQC, solid and coarsely QH maps 84

8. Mapping problems 90

9. Neargeodesics and coarse length 96

10. Uniform domains 101

11. Maps of uniform domains 108

12. Further results 113

13. Open problems 115

References 116

Index 118

\section{Introduction}

1.1. Quasiconformality in $R^{n}$. Quasiconformal maps in the plane $R^{2}$ were introduced by H. Grötzsch in 1928. He considered diffeomorphisms $f: G \rightarrow G^{\prime}$ between domains in $R^{2}$ such that the image of each infinitesimal circle is an infinitesimal ellipse with semiaxes $a \leq b$ such that $b / a$ is bounded by a constant $K$. The concept was later generalized to the euclidean space $R^{n}, n \geq 2$, by several authors. However, it turned out that it is not

1991 Mathematics Subject Classification: Primary 30C65; Secondary 46B20.

The paper is in final form and no version of it will be published elsewhere. 
convenient to assume differentiability everywhere. The basic theory of quasiconformal maps in $R^{n}$ is given in the author's book [V $\left.\ddot{a}_{2}\right]$. The case $n=2$ has several special features; it is treated in the book $[\mathrm{LV}]$ of O. Lehto and K. I. Virtanen.

There are plenty of mutually equivalent definitions for quasiconformality in $R^{n}$. In this article we adopt the following simplified version of the metric definition. Let $n \geq 2$, let $G$ and $G^{\prime}$ be domains in $R^{n}$, and let $f: G \rightarrow G^{\prime}$ be a homeomorphism. For $x \in G$, the metric dilatation (also called the linear dilatation) of $f$ at $x$ is the number $H(x, f) \in[1, \infty]$ defined by

$$
H(x, f)=\limsup _{r \rightarrow 0} \frac{\sup \{|f y-f x|:|y-x|=r\}}{\inf \{|f y-f x|:|y-x|=r\}} .
$$

For $1 \leq K<\infty$, we say that $f$ is $K$-quasiconformal if $H(x, f) \leq K$ for all $x \in G$, and that $f$ is quasiconformal if it is $K$-quasiconformal for some $K$.

In the literature, it is customary to only assume that $H(x, f)$ is bounded by some constant everywhere and by $K$ almost everywhere. Moreover, one can introduce the so-called inner and outer dilatations, which have several pleasant properties concerning composition, inverse maps and limits. In particular, the definition for $K$-quasiconformality in [Vä 2 ] differs from the above, but both definitions give the same class of quasiconformal maps. More precisely, a $K$-quasiconformal map in the above sense is $K^{n-1}$-quasiconformal in the sense of [V $\left.\ddot{a}_{2}\right]$, and a $K$-quasiconformal map in the sense of [V $\ddot{a}_{2}$ ] is $K^{\prime}$-quasiconformal in the above sense with some $K^{\prime}=K^{\prime}(K, n)$.

The metric definition given above is natural and easy to state, but it has the disadvantage that one cannot easily prove the properties of quasiconformal maps starting from it. For example, the inverse map of a $K$-quasiconformal map is $K^{\prime}$-quasiconformal with $K^{\prime}=$ $K^{\prime}(K, n)$ and the composition of a $K_{1}$-quasiconformal map and a $K_{2}$-quasiconformal map is $K^{\prime \prime}$-quasiconformal with $K^{\prime \prime}=K^{\prime \prime}\left(K_{1}, K_{2}, n\right)$. These properties are obvious if the geometric definition [V $\left.\ddot{a}_{2}, 13.1\right]$ is used. Indeed, then $K^{\prime}=K$ and $K^{\prime \prime}=K_{1} K_{2}$. But only recently, J. Heinonen and P. Koskela $[\mathrm{HK}]$ have given a direct proof based on the metric definition.

We emphasize that this definition is only used in $R^{n}$. In general Banach spaces we shall adopt a different definition.

1.3. Setting. Throughout this article, $E$ and $E^{\prime}$ will always denote real Banach spaces. Unless otherwise stated, we assume that their dimensions are at least two. Furthermore, $G \subset E$ and $G^{\prime} \subset E^{\prime}$ will always be domains (open connected nonempty sets). We shall mainly be interested in homeomorphisms $f: G \rightarrow G^{\prime}$.

A fundamental question is: When should such a map be called quasiconformal? Our definition (see 4.3) will be based on the quasihyperbolic metric, but we shall give alternative characterizations based on local quasisymmetry and on distortion of spherical rings. In order to make a distinction from the definition given in 1.1, we shall call these maps freely quasiconformal. The word "free" refers to "dimension-free" and also to "volumefree". In the case $E=E^{\prime}=R^{n}$, this definition is equivalent to the definition of 1.1. In the general case, the definition (1.2) of the metric dilatation $H(x, f)$ still makes sense, and our definition for free quasiconformality implies that $H(x, f)$ is bounded. Whether the converse is true, is an important open problem. 
In the study of free quasiconformality, several other classes of maps arise naturally, namely solid, quasihyperbolic and coarsely quasihyperbolic maps. I believe that these classes have independent interest. By the free quasiworld we mean the study of all these maps.

The free quasiworld has been considered in the four papers [Vä5,6,7,9] of the author since 1990. Related work was done by G. Porru $\left[\mathrm{Po}_{1,2}\right]$ and P. Caraman [Ca] in the late seventies. The purpose of this article is to be a "mini monograph" on the free quasiworld by giving a fairly self-contained exposition of the basic concepts and results of this theory. Most of the material is from [V $\left.\ddot{a}_{5,6,7,9}\right]$.

These papers will be cited as I, II, III, IV. For example, [II, 6.12] means the result 6.12 in $\left[V \ddot{a}_{6}\right]$. We also give some background material on Banach spaces and on quasisymmetric and quasimöbius maps.

Several open problems are stated in the text. These are called problems, and a list of them is given in Section 13.

1.4. Comparison with the case $E=R^{n}$. A number of methods and tools, useful in $R^{n}$, are not available in an infinite-dimensional Banach space $E$ :

1. The space $E$ is not locally compact, and its one-point extension $\dot{E}=E \cup\{\infty\}$ is not compact. Normal family arguments are therefore not valid in $E$, and many extremal problems have no solution.

2. Several intuitively obvious topological properties of $R^{n}$ are not valid in $E$. For example, a ball $B$ is homeomorphic to the domain $A$ between two concentric spheres. However, it turns out that some strange-looking topological phenomena do not occur in the quasiworld. In particular, there is no freely quasiconformal map of $B$ onto $A$; see 8.5.

3. There is no natural measure in $E$ like the Lebesgue measure in $R^{n}$. Balls have no volume. The method of moduli of path families is useless in the free quasiworld.

4. Packing arguments fail in $E$.

5. Domains in $E$ have no Whitney decomposition.

1.5. Motivation. I have been asked whether there is any good reason to study the free quasiworld. Personally, I needed no reason, only the feeling that this study is natural, interesting and fun. However, I try to give some motivation.

1. Any generalization of a well-studied topic (quasiconformality in $R^{n}$ ) usually gives a better understanding of the old theory. For example, we see which properties of the quasiconformal maps in $R^{n}$ depend only on distance and not on volume.

2. To develop the theory we must find new methods and tools. Our proofs work also in the classical case, and hence we get new proofs for old results.

3. Several well-known results can be formulated in a new dimension-free manner.

4. The theory gives rise to the study of various mapping classes related to quasiconformality.

5. The theory gives rise to questions about quasiconformal equivalence between Banach spaces and suggests connections between the quasiworld and functional analysis. At the time of this writing, there are hardly any results in this direction. 
1.6. Notation. In addition to the Banach spaces $E$ and $E^{\prime}$, we sometimes work in arbitrary metric spaces $X$ and $Y$. Unless otherwise stated, we let $|a-b|$ denote the distance between the points $a$ and $b$ in any metric space. Balls and spheres are written as

$$
B(a, r)=\{x:|x-a|<r\}, \bar{B}(a, r)=\{x:|x-a| \leq r\}, S(a, r)=\{x:|x-a|=r\} .
$$

In a Banach space $E$, we may omit the center $a$ if it is the origin. In particular, $B(1)$ is the open unit ball of $E$.

The norm of a vector $x \in E$ is written as $|x|$, the diameter of a set $A \subset E$ as $d(A)$, and the distance between nonempty sets $A, B \subset E$ as $d(A, B)$. The one-point extension of $E$ is the Hausdorff space $\dot{E}=E \cup\{\infty\}$, where the neighborhoods of $\infty$ are the complements of closed bounded sets of $E$. The boundary $\partial A$ and the closure $\bar{A}$ of a set $A \subset E$ are taken in $\dot{E}$. The closed line segment with endpoints $a, b \in E$ is $[a, b]$. For a half open segment we use the obvious notation $[a, b)$. To simplify expressions we often omit parentheses writing $f x$ instead of $f(x)$, etc. We let $l(\gamma)$ denote the length of an arc or a Jordan curve $\gamma$. If $x$ and $y$ are points of an arc $\gamma$, then $\gamma[x, y]$ is the subarc of $\gamma$ between $x$ and $y$. For real numbers $a, b$ we write

$$
a \vee b=\max (a, b), a \wedge b=\min (a, b) .
$$

We let $\mathbf{N}, \mathbf{Z}$ and $\mathbf{R}$ denote the set of positive integers, integers and real numbers, respectively.

1.7. Prerequisites. Very little mathematical background is needed for reading this article. We only assume that the reader is familiar with the elements of set-theoretical topology, metric spaces, linear algebra, Banach spaces and Hilbert spaces. No previous knowledge on finite-dimensional quasiconformality is required, but of course, such a knowledge is useful in giving motivation for this theory. We do not need measure and integration theory beyond the integral of a continuous function of one real variable.

Acknowledgments. I thank Pekka Alestalo for reading the manuscript and for valuable comments.

\section{Uniform continuity and quasiconvexity}

2.1. Summary. We begin each section with a brief summary of the section. In this section we present some elementary concepts and results on metric spaces, needed in Section 4. We also prove the quasiconvexity of spheres in Banach spaces.

2.2. Definitions. Let $X$ and $Y$ be metric spaces. A map $f: X \rightarrow Y$ is uniformly continuous if there is $t_{0} \in(0, \infty]$ and a homeomorphism $\varphi:\left[0, t_{0}\right) \rightarrow[0, \infty)$ such that

$$
|f x-f y| \leq \varphi(|x-y|) \quad \text { for all } x, y \in X \text { with }|x-y|<t_{0} \text {. }
$$

The function $\varphi$ is a modulus of continuity of $f$, and we say that $f$ is $\left(\varphi, t_{0}\right)$-uniformly continuous. If $t_{0}=\infty$, we briefly say that $f$ is $\varphi$-uniformly continuous.

The fact that $\varphi$ is a homeomorphism means that $\varphi$ is continuous and strictly increasing, $\varphi(0)=0$, and $\varphi(t) \rightarrow \infty$ as $t \rightarrow t_{0}$. We assumed this for the sake of convenience, but it is sufficient that $(2.3)$ holds for some function $\varphi:\left[0, t_{0}\right) \rightarrow[0, \infty)$ such that $\varphi$ is bounded 
on each interval $[0, t], t<t_{0}$, and $\varphi(t) \rightarrow 0$ as $t \rightarrow 0$, because one can then replace $\varphi$ by a homeomorphism $\psi:\left[0, t_{0}\right) \rightarrow[0, \infty)$ with $\varphi(t) \leq \psi(t)$. This can be found, for example, as follows: Choose numbers $r_{j} \in\left(0, t_{0}\right), j \in \mathbf{Z}$, such that $r_{j}<r_{j+1}, r_{j} \rightarrow 0$ as $j \rightarrow-\infty$, and $r_{j} \rightarrow t_{0}$ as $j \rightarrow \infty$. Set $a_{j+1}=\sup \left\{\varphi(t): 0 \leq t \leq r_{j}\right\}$. Define $\varphi_{1}:\left[0, t_{0}\right) \rightarrow[0, \infty)$ by $\varphi_{1}\left(r_{j}\right)=a_{j+1}$ and letting $\varphi_{1} \mid\left[r_{j}, r_{j+1}\right]$ be affine for each $j$. Choose some homeomorphism $\varphi_{2}:\left[0, t_{0}\right) \rightarrow[0, \infty)$. Then $\psi(t)=\varphi_{1}(t)+\varphi_{2}(t)$ is the desired function.

The linear function $\varphi(t)=M t, M>0$, gives the Lipschitz maps: $f$ is $M$-Lipschitz if

$$
|f x-f y| \leq M|x-y| \quad \text { for all } x, y \in X .
$$

More generally, if $C \geq 0$ and

$$
|f x-f y| \leq M|x-y|+C \quad \text { for all } x, y \in X,
$$

we say that $f$ is $C$-coarsely $M$-Lipschitz. If

$$
|x-y| / M \leq|f x-f y| \leq M|x-y| \quad \text { for all } x, y \in X,
$$

then $f$ is $M$-bilipschitz. Finally, if

$$
(|x-y|-C) / M \leq|f x-f y| \leq M|x-y|+C \quad \text { for all } x, y \in X,
$$

the map $f$ is $C$-coarsely $M$-bilipschitz.

A metric space $X$ is $c$-quasiconvex, $c \geq 1$, if each pair of points $a, b \in X$ can be joined by an arc $\gamma$ of length $l(\gamma) \leq c|a-b|$. For example, a convex subset of a Banach space is 1-quasiconvex. An $\operatorname{arc} \gamma$ is $c$-quasiconvex if and only if $l(\gamma[x, y]) \leq c|x-y|$ for all $x, y \in \gamma$.

The following result gives a useful sufficient condition for a map of a quasiconvex space to be coarsely Lipschitz.

2.3. Lemma. Suppose that $X$ is c-quasiconvex, that $q>0, C \geq 0$, and that $f: X \rightarrow Y$ is a map such that $|f x-f y| \leq C$ whenever $|x-y| \leq q$. Then

$$
|f x-f y| \leq(c C / q)|x-y|+C \quad \text { for all } x, y \in X \text {. }
$$

Proof. Given $x, y \in X$, we choose an arc $\gamma$ from $x$ to $y$ with $l(\gamma) \leq c|x-y|$. Let $k \geq 0$ be the unique integer satisfying $k q<l(\gamma) \leq(k+1) q$. Then we can choose successive points $x=x_{0}, \ldots, x_{k+1}=y$ of $\gamma$ such that $l\left(\gamma\left[x_{j-1}, x_{j}\right]\right) \leq q$ for all $1 \leq j \leq k+1$. Since $\left|x_{j-1}-x_{j}\right| \leq q$, we have $\left|f x_{j-1}-f x_{j}\right| \leq C$ for all $1 \leq j \leq k+1$. Hence

$$
|f x-f y| \leq \sum_{j=1}^{k+1}\left|f x_{j}-f x_{j-1}\right| \leq(k+1) C .
$$

Since $k<l(\gamma) / q \leq c|x-y| / q$, this proves the lemma.

2.4. Quantitativeness. In the results concerning the quasiworld, it is usually important to know which quantities depend on which quantities. Let $A$ be a condition with data $v$ and let $A^{\prime}$ be a condition with data $v^{\prime}$. We say that $A$ implies $A^{\prime}$ quantitatively if $A$ implies $A^{\prime}$ so that $v^{\prime}$ depends only on $v$. If $A$ and $A^{\prime}$ imply each other quantitatively, we say that they are quantitatively equivalent.

For example, the condition of Lemma 2.3 implies quantitatively that $f$ is $C$-coarsely $M$-Lipschitz, since $C$ and $M=c C / q$ depend only on the given numbers $c, C$ and $q$. In the following result, if (1) is true with given $t_{0}$ and $\varphi$, then (2) is true with another function 
$\varphi_{1}$ depending only on the pair $\left(\varphi, t_{0}\right)$, and (3) is true with a function $\varphi_{1}$ and numbers $M, C$ depending only on $\left(\varphi, t_{0}\right)$. A symbol (like $\varphi$ here) appearing in both $A$ and $A^{\prime}$ need not have the same value in both conditions.

2.5. Theorem. Let $X$ be c-quasiconvex and let $f: X \rightarrow Y$ be a map. Then the following conditions are quantitatively equivalent:

(1) $f$ is $\left(\varphi, t_{0}\right)$-uniformly continuous,

(2) $f$ is $\varphi$-uniformly continuous,

(3) $f$ is $\varphi$-uniformly continuous and there are $M>0$ and $C \geq 0$ such that $\varphi(t) \leq$ $M t+C$.

Proof. Trivially $(3) \Rightarrow(2) \Rightarrow(1)$. Assume that $(1)$ is true. Choose a number $q \in$ $\left(0, t_{0}\right)$, for example, $q=1 \wedge\left(t_{0} / 2\right)$. Then the condition of Lemma 2.3 is true with this $q$ and with $C=\varphi(q)$. Hence $|f x-f y| \leq M|x-y|+C$ for $M=c C / q$ and for all $x, y \in X$. We see that $(3)$ is true with any homeomorphism $\varphi_{1}:[0, \infty) \rightarrow[0, \infty)$ such that $\varphi(t) \leq \varphi_{1}(t)$ for $0 \leq t \leq q$ and $\varphi_{1}(t)=M t+C$ for $t \geq q$. For example, we may set

$$
\varphi_{1}(t)=\varphi(t)+2(\varphi(q)-\varphi(q / 2)) t / q \quad \text { for } 0 \leq t \leq q / 2,
$$

$\varphi_{1}(t)=M t+C$ for $t \geq q$, and let $\varphi_{1}$ be affine on $[q / 2, q]$.

2.6. Theorem. If $\operatorname{dim} E=2$, then $l(S(1)) \leq 8$.

Proof. We introduce an arbitrary euclidean metric in $E$ in order to consider areas. By compactness we can find points $a, b \in S(1)$ such that the area of the triangle with vertices $0, a, b$ is maximal. Then $\bar{B}(1)$ lies in the parallelogram with vertices $\pm a \pm b$, since otherwise it is easy to find a larger triangle.

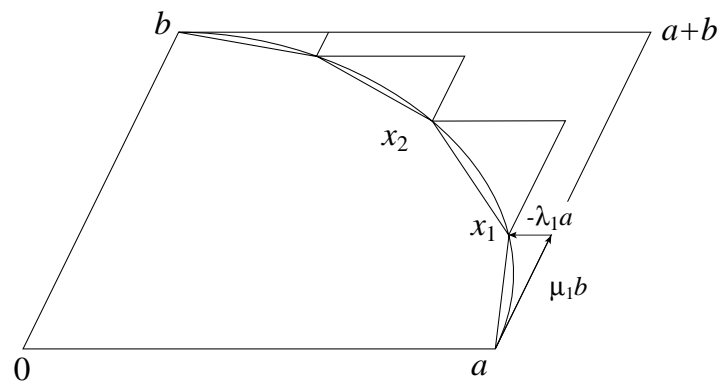

Fig. 1

The points $\pm a$ and $\pm b$ divide $S(1)$ into four arcs. We show that each of these has length at most 2. It suffices to consider the arc $\gamma$ between $a$ and $b$. Let $a=x_{0}, \ldots, x_{m}=b$ be successive points of $\gamma$. For each $1 \leq j \leq m$ we can write $x_{j}-x_{j-1}=-\lambda_{j} a+\mu_{j} b$ for some $\lambda_{j} \geq 0, \mu_{j} \geq 0$. Then $\lambda_{1}+\ldots \lambda_{m}=\mu_{1}+\ldots+\mu_{m}=1$; see Figure 1 . We obtain

$$
\sum_{j=1}^{m}\left|x_{j-1}-x_{j}\right| \leq \sum_{j=1}^{m}\left(\left|\lambda_{j} a\right|+\left|\mu_{j} b\right|\right)=|a|+|b|=2,
$$

and hence $l(\gamma) \leq 2$. 
2.8. Notes. Theorem 2.6 was proved by S. Gołąb [Go] in 1932 and rediscovered by D. Laugwitz [Lau, 1.1] in 1954. One can show that a sphere in $E$ is always 2-quasiconvex [Sc $2,4 \mathrm{G}, \mathrm{p} .17]$. The bound 8 in 2.6 is sharp, and it is obtained when $S(1)$ is a parallelogram. The smallest possible value for $l(S(1))$ is 6 ; this occurs if $S(1)$ is a regular hexagon. See the book $\left[\mathrm{Sc}_{2}\right]$ of Schäffer for these and related topics.

\section{Quasihyperbolic metric}

3.1. Summary. The quasihyperbolic metric of a domain $G \varsubsetneqq E$ is one of the key tools in the free quasiworld. In particular, the definition of a freely quasiconformal map in Section 4 will be based on the quasihyperbolic metric. In this section we give the definition and some basic properties of this metric.

3.2. Definitions. Let $G \varsubsetneqq E$ be a domain. For $x \in G$ we let $\delta_{G}(x)$ denote the distance $d(x, \partial G)$. This notation will be used throughout this article, and we shall usually abbreviate $\delta(x)=\delta_{G}(x), \delta^{\prime}(x)=\delta_{G^{\prime}}(x)$.

Let $\gamma \subset G$ be a rectifiable arc of length $\lambda=l(\gamma)$ with endpoints $a$ and $b$. Then $\gamma$ has an arclength parametrization $\gamma^{0}:[0, \lambda] \rightarrow \gamma$ such that $\gamma^{0}(0)=a, \gamma^{0}(\lambda)=b$, and $l\left(\gamma\left[a, \gamma^{0}(t)\right]\right)=t$ for all $t \in[0, \lambda]$; see $\left[\mathrm{V} \ddot{a}_{2}, \S 2\right]$. The path $\gamma^{1}(t)=\gamma^{0}(\lambda-t)$ gives another arclength parametrization with $\gamma^{1}(0)=b, \gamma^{1}(\lambda)=a$. In most cases it does not matter which one we choose, and either of them can be written as $\gamma^{0}$.

If $\gamma$ is rectifiable and if $u: \gamma \rightarrow \mathbf{R}$ is continuous, the line integral of $u$ along $\gamma$ is defined as

$$
\int_{\gamma} u(x)|d x|=\int_{0}^{\lambda} u\left(\gamma^{0}(t)\right) d t .
$$

The quasihyperbolic length of $\gamma$ in $G$ is the number

$$
l_{k}(\gamma)=\int_{\gamma} \frac{|d x|}{\delta(x)} .
$$

We shall often abbreviate "quasihyperbolic" as QH.

Let $a$ and $b$ be points in $G$. We can always join $a$ and $b$ by a rectifiable $\operatorname{arc}$ in $G$, for example, by a broken line. In the case $a=b$, we consider the singleton $\{a\}$ as an arc of $\mathrm{QH}$ length zero. The quasihyperbolic $(\mathrm{QH})$ distance between $a$ and $b$ in $G$ is the number

$$
k_{G}(a, b)=\inf _{\gamma} l_{k}(\gamma)
$$

over all arcs $\gamma$ joining $a$ and $b$ in $G$. We shall usually abbreviate $k=k_{G}, k^{\prime}=k_{G^{\prime}}$.

It is easy to verify that $k$ is a metric in $G$, called the quasihyperbolic metric of $G$.

3.3. Convention. Whenever a statement involves the $\mathrm{QH}$ metric $k_{G}$ or the distance $\delta_{G}(x)$, we shall tacitly assume that the domain $G$ is not the whole space.

3.4. TheOREM. (1) $l_{k}(\gamma)$ is the length of $\gamma$ in the metric space $(G, k)$.

(2) The space $(G, k)$ is c-quasiconvex for all $c>1$. 
Proof. (1) Let $x_{0}, \ldots, x_{m}$ be successive points of $\gamma$. Then

$$
\sum_{j=1}^{m} k\left(x_{j-1}, x_{j}\right) \leq \sum_{j=1}^{n} l_{k}\left(\gamma\left[x_{j-1}, x_{j}\right]\right) \leq l_{k}(\gamma) .
$$

Hence the $k$-length of $\gamma$ is at most $l_{k}(\gamma)$.

To prove the reverse inequality, let $\lambda=l(\gamma)$ and let $\gamma^{0}:[0, \lambda] \rightarrow \gamma$ be the arclength parametrization of $\gamma$. Then

$$
l_{k}(\gamma)=\int_{0}^{\lambda} g(t) d t
$$

where $g(t)=1 / \delta\left(\gamma^{0}(t)\right)$. Let $0<\varepsilon<1 / 2$. Since $g$ is continuous, a simple compactness argument shows that there is a subdivision $0=t_{0}<t_{1}<\ldots<t_{m}=\lambda$ of $[0, \lambda]$ such that setting $x_{i}=\gamma^{0}\left(t_{i}\right)$ and $\gamma_{i}=\gamma\left[x_{i-1}, x_{i}\right]$ we have

$$
l_{k}(\gamma) \leq \sum_{j=1}^{m} g\left(t_{i}\right)\left(t_{i}-t_{i-1}\right)+\varepsilon
$$

and $\gamma_{i} \subset B\left(x_{i}, \varepsilon \delta\left(x_{i}\right)\right)$ for all $1 \leq i \leq m$. For each $1 \leq i \leq m$ we choose successive points $x_{i-1}=x_{i 0}, x_{i 1}, \ldots, x_{i, m_{i}}=x_{i}$ of $\gamma_{i}$ such that

$$
l\left(\gamma_{i}\right) \leq \sum_{j=1}^{m_{i}}\left|x_{i, j-1}-x_{i j}\right|+\varepsilon / m
$$

With the aid of the estimate $3.7(4)$ below, we get

$$
\frac{\left|x_{i, j-1}-x_{i j}\right|}{\delta\left(x_{i}\right)} \leq(1+2 \varepsilon) k\left(x_{i, j-1}, x_{i j}\right)
$$

for all $i$ and $j$. Since $t_{i}-t_{i-1}=l\left(\gamma_{i}\right)$ and since $g\left(t_{i}\right)=1 / \delta\left(x_{i}\right)$, these estimates imply

$$
l_{k}(\gamma) \leq(1+2 \varepsilon) \sum_{i} \sum_{j} k\left(x_{i, j-1}, x_{i j}\right)+2 \varepsilon .
$$

Here the double sum is at most the $k$-length of $\gamma$. Since $\varepsilon$ is arbitrary, this yields the desired inequality.

(2) Let $a \neq b$ be points in $G$ and let $c>1$. Choose an arc $\gamma$ joining $a$ and $b$ with $l_{k}(\gamma)<$ $c k(a, b)$. By $(1)$, the $k$-length of $\gamma$ is less than $c k(a, b)$. Hence $(G, k)$ is $c$-quasiconvex.

3.5. REMARK. It is natural to ask whether 3.4(2) holds with $c=1$. In other words, is it always possible to join two points $a, b \in G$ by a $\mathrm{QH}$ geodesic? In $R^{n}$, a positive answer was given by F. W. Gehring and B.G. Osgood [GO, Lemma 1] in 1979. In the general case the answer is negative. A counterexample is given in [I, 2.9]. We give another example, due to P. Alestalo (unpublished).

Let $E$ be the Hilbert space $l_{2}$ of all sequences $x=\left(x_{1}, x_{2}, \ldots\right)$ such that $|x|^{2}=$ $x_{1}^{2}+x_{2}^{2}+\ldots<\infty$. Let $Q \subset E$ be the Hilbert cube $\left\{x \in E: x_{1}=0,\left|x_{j}\right| \leq 1 / j\right.$ for $j \geq 2\}$, and let $G$ be the domain $E \backslash Q$. We show that the points $e_{1}=(1,0,0, \ldots)$ and $-e_{1}$ cannot be joined by a $\mathrm{QH}$ geodesic in $G$.

Let $\gamma \subset G$ be a rectifiable arc with endpoints $e_{1}$ and $-e_{1}$. Setting

$$
A x=\left(x_{1}, 0, x_{2}, x_{3}, \ldots\right)
$$


we obtain an isometric embedding $A: E \rightarrow E$. Then also $A \gamma$ joins $e_{1}$ and $-e_{1}$ in $G$. We show that $l_{k}(A \gamma)<l_{k}(\gamma)$, and hence $\gamma$ cannot be a QH geodesic. For this it suffices to show that $\delta(A x) \geq \delta(x)$ for all $x \in G$ and that $\delta(A x)>\delta(x)$ for some $x \in \gamma$.

Let $x \in G$. Since $Q=\partial G$ is compact, there is $y \in Q$ with $|A x-y|=\delta(A x)$. Since $(A x)_{2}=0$, we have $y_{2}=0$, and hence $y=A z$ for $z=\left(y_{1}, y_{3}, y_{4}, \ldots\right)$. Since $z \in Q$, we have $\delta(x) \leq|x-z|=|A x-y|=\delta(A x)$ for all $x \in G$.

Since $\gamma$ joins $e_{1}$ and $-e_{1}$, there is $x \in \gamma$ with $x_{1}=0$. Since $x \notin Q$, there is $j \geq 2$ with $\left|x_{j}\right|>1 / j$. We may assume that $x_{j}>1 / j$. Let $y$ and $z$ be as above, and let $u \in E$ be the point with $u_{j}=1 / j$ and $u_{i}=z_{i}$ for $i \neq j$. Then $u \in Q$. Since $\left|z_{j}\right|=\left|y_{j+1}\right| \leq 1 /(j+1)$, we have $\left|x_{j}-z_{j}\right|>\left|x_{j}-u_{j}\right|$, and hence $|x-z|>|x-u|$. Consequently,

$$
\delta(x) \leq|x-u|<|x-z|=|A x-y|=\delta(A x) .
$$

3.6. Other distances. Let $G \varsubsetneqq E$ and let $a, b \in G$. In addition to the $\mathrm{QH}$ distance $k(a, b)=k_{G}(a, b)$, it is sometimes convenient to consider the numbers

$$
r_{G}(a, b)=\frac{|a-b|}{\delta(a) \wedge \delta(b)}, j_{G}(a, b)=\log \left(1+r_{G}(a, b)\right) .
$$

The relative distance $r_{G}$ does not define a metric in $G$, but the logarithmic distance $j_{G}$ does. However, we shall not need this fact.

We next give some useful estimates for the QH distance. Part (4) of the following result was already used in the proof of 3.4(1).

3.7. Theorem. (1) $k_{G}(a, b) \geq j_{G}(a, b) \geq \log (\delta(b) / \delta(a))$ for all $a, b \in G$.

(2) $|a-b| \leq\left(e^{k(a, b)}-1\right) \delta(a) \leq e^{k(a, b)} \delta(a)$ for all $a, b \in G$.

(3) If $a \in G, 0<t<1$, and $x, y \in \bar{B}(a, t \delta(a))$, then

$$
k(x, y) \leq \frac{1}{1-t} \frac{|x-y|}{\delta(a)} .
$$

(4) If, in addition, $t \leq 1 / 2$, then

$$
k(x, y) \geq \frac{1}{1+2 t} \frac{|x-y|}{\delta(a)} .
$$

(5) If $a, b \in G$ with $|a-b| / \delta(a)=q<1$, then

$$
k(a, b) \leq \log \frac{1}{1-q} .
$$

Proof. (1) Assume that $\gamma$ is a rectifiable arc joining $a$ to $b$ in $G$. Let $\gamma^{0}:[0, \lambda] \rightarrow \gamma$ be the arclength parametrization of $\gamma$ with $\gamma^{0}(0)=a$. For each $s \in[0, \lambda]$ we have

$$
\delta\left(\gamma^{0}(s)\right) \leq \delta(a)+\left|\gamma^{0}(s)-a\right| \leq \delta(a)+l\left(\gamma\left[a, \gamma^{0}(s)\right]\right)=\delta(a)+s .
$$

Hence

$$
l_{k}(\gamma) \geq \int_{0}^{\lambda} \frac{d s}{\delta(a)+s}=\log \left(1+\frac{\lambda}{\delta(a)}\right) \geq \log \left(1+\frac{|a-b|}{\delta(a)}\right) .
$$

Since $a$ can be replaced by $b$, we obtain the first inequality of (1). Since $\delta(b) \leq \delta(a)+|a-b|$, the second inequality follows.

Part (2) follows from (1). 
Since $\delta(z) \geq(1-t) \delta(a)$ for all $z \in[x, y]$, (3) follows by integration along the line segment $[x, y]$.

To prove (4), assume that $\gamma$ joins $x$ and $y$ in $G$. If $\gamma \subset B(a, 2 t \delta(a))$, then $\delta(z) \leq$ $(1+2 t) \delta(a)$ for all $z \in G$, and hence

$$
l_{k}(\gamma) \geq \frac{|x-y|}{(1+2 t) \delta(a)} .
$$

If $\gamma \not \subset B(a, 2 t \delta(a))$, then $\gamma$ has nonoverlapping subarcs $\gamma_{1}, \gamma_{2}$ joining the boundary components of the $\operatorname{ring} A=\{z: t \delta(a) \leq|z-a| \leq 2 t \delta(a)\}$ in $A$. For $z \in \gamma_{j}$ we have $\delta(z) \leq(1+2 t) \delta(a)$. Since $l\left(\gamma_{j}\right) \geq t \delta(a) \geq|x-y| / 2$, we again obtain (3.8). This proves (4).

Part (5) follows by integration along $[a, b]$.

3.9. Theorem. Suppose that $a, b \in G$ and that either $|a-b| \leq \delta(a) / 2$ or $k(a, b) \leq 1$. Then

$$
\frac{1}{2} \frac{|a-b|}{\delta(a)} \leq k(a, b) \leq 2 \frac{|a-b|}{\delta(a)} .
$$

Proof. If $|a-b| \leq \delta(a) / 2$, this follows from parts (3) and (4) of 3.7. Suppose that $k(a, b)=r \leq 1$. By $3.7(2)$ we have

$$
|a-b| \leq\left(e^{r}-1\right) \delta(a) \leq 2 r \delta(a)
$$

which yields the first inequality of the theorem. If $|a-b| \geq \delta(a) / 2$ and $k(a, b) \leq 1$, the second inequality is trivially true.

3.10. Corollary. The QH metric and the norm metric define the same topology of $G \subsetneq E$.

3.11. Diameters. If $X$ is a set and if $\varrho: X \times X \rightarrow[0, \infty)$ is a function, the $\varrho$-diameter of a set $A \subset X$ is

$$
\varrho(A)=\sup \{\varrho(x, y): x \in A, y \in A\}
$$

with $\varrho(\varnothing)=0$. In particular, if $A \subset G \subsetneq E$, we can consider the $\mathrm{QH}$ diameter $k(A)=$ $k_{G}(A)$ and the relative diameter $r_{G}(A)$; see 3.6. Moreover, we define the relative size of $A$ as

$$
\bar{r}_{G}(A)=\frac{d(A)}{d(A, \partial G)} .
$$

If $d(A, \partial G)=0$, we set $\bar{r}_{G}(A)=\infty$. We give some inequalities for these numbers.

3.12. Theorem. (1) $r_{G}(A) \leq \bar{r}_{G}(A) \leq 2 r_{G}(A)$.

(2) $k_{G}(A) \geq \log \left(1+r_{G}(A)\right) \geq \log \left(1+\bar{r}_{G}(A) / 2\right)$.

(3) If $k_{G}(A)<\infty$, then $d(A, \partial G)>0$.

Proof. The first inequality of (1) is clear. To prove the second one, let $a, b, c \in A$. Then

$$
\frac{|a-b|}{\delta(c)} \leq \frac{|a-c|}{\delta(c)}+\frac{|c-b|}{\delta(c)} \leq r_{G}(a, c)+r_{G}(c, b) \leq 2 r_{G}(A),
$$

and hence $\bar{r}_{G}(A) \leq 2 r_{G}(A)$.

Part (2) follows directly from (1) and from 3.7(1), and (3) follows from (2). 
3.13. Notes. The quasihyperbolic metric of a domain $G \subsetneq R^{n}$ was introduced by F. W. Gehring and B. P. Palka $[\mathrm{GP}]$ in 1976. In the case where $G$ is a half space in $R^{n}$ it agrees with the classical hyperbolic metric. This explains the terminology. The distance $j_{G}(a, b)$ in 3.6 is a modification of $\mathrm{M}$. Vuorinen $[\mathrm{Vu}]$ of the distance

$$
j_{G}^{\prime}(a, b)=\frac{1}{2} \log \left[\left(1+\frac{|a-b|}{\delta(a)}\right)\left(1+\frac{|a-b|}{\delta(b)}\right)\right],
$$

considered in $[\mathrm{GO}]$. A related expression was used by P. W. Jones [ $\left.\mathrm{J}_{1}\right]$. We have always $j_{G} \leq j_{G}^{\prime} \leq 2 j_{G}$.

\section{Maps in the free quasiworld}

4.1. Summary. We introduce the central objects of the free quasiworld: solid, freely quasiconformal, quasihyperbolic and coarsely quasihyperbolic maps.

4.2. Fullness. We recall that $G$ and $G^{\prime}$ are always domains in Banach spaces $E$ and $E^{\prime}$, respectively. Suppose that $f: G \rightarrow G^{\prime}$ is a homeomorphism. For each subdomain $D \subset G$, we let $f_{D}: D \rightarrow f D$ denote the homeomorphism defined by $f$. We say that $f$ has fully a property $P$ if $f_{D}$ has property $P$ for each proper subdomain $D \subsetneq G$.

4.3. Definitions. Let $f: G \rightarrow G^{\prime}$ be a homeomorphism. We say that $f$ is $\varphi$-solid if $f$ and $f^{-1}$ are $\varphi$-uniformly continuous in the $\mathrm{QH}$ metrics $k=k_{G}$ and $k^{\prime}=k_{G^{\prime}}$; see 2.2 . In other words, $\varphi:[0, \infty) \rightarrow[0, \infty)$ is a homeomorphism and

$$
\varphi^{-1}(k(x, y)) \leq k^{\prime}(f x, f y) \leq \varphi(k(x, y)) \text { for all } x, y \in G .
$$

Observe that the definition makes sense only if $G \neq E$ and $G^{\prime} \neq E^{\prime}$.

The special case $\varphi(t)=M t, M \geq 1$, gives the quasihyperbolic maps. More precisely, $f$ is called $M$-quasihyperbolic or $M$-QH if

$$
k(x, y) / M=k^{\prime}(f x, f y) \leq M k(x, y) \quad \text { for all } x, y \in G .
$$

In other words, $f$ is $M$-bilipschitz in the $\mathrm{QH}$ metric.

If $f$ is $C$-coarsely $M$-bilipschitz in the $\mathrm{QH}$ metric, then $f$ is said to be $C$-coarsely $M$-quasihyperbolic. This means that

$$
(k(x, y)-C) / M \leq k^{\prime}(f x, f y) \leq M k(x, y)+C \quad \text { for all } x, y \in G .
$$

Finally, a homeomorphism $f: G \rightarrow G^{\prime}$ is freely $\varphi$-quasiconformal or $\varphi$-FQC if $f$ is fully $\varphi$-solid, that is, the homeomorphism $f_{D}: D \rightarrow f D$ is $\varphi$-solid for each subdomain $D \subsetneq G$. Contrary to the classes defined above, this definition makes sense also if $G=E$ or $G^{\prime}=E^{\prime}$.

4.7. Theorem. (1) If $f: G \rightarrow G^{\prime}$ is $\varphi$-solid, then $f$ is $C$-coarsely $M-\mathrm{QH}$ with any $C>0$ and with $M=C / \varphi^{-1}(C)$.

(2) If $G \neq E, G^{\prime} \neq E^{\prime}$ and if $f: G \rightarrow G^{\prime}$ is $\varphi$-FQC, then $f$ is $\varphi$-solid.

Proof. Part (1) follows directly from 2.3 and 3.4(2). To prove (2) let $a, b \in G$. Choose a sequence $\left(x_{j}\right)$ in $G \backslash\{a, b\}$ such that $\delta\left(x_{j}\right) \rightarrow 0$. Write $D_{j}=G \backslash\left\{x_{j}\right\}$. Then

$$
k^{\prime}(f a, f b) \leq k_{f D_{j}}(f a, f b) \leq \varphi\left(k_{D_{j}}(a, b)\right) .
$$


Since $\delta_{D_{j}}(x) \rightarrow \delta(x)$ uniformly on each arc in $G$, it is easy to see that $k_{D_{j}}(a, b) \rightarrow k(a, b)$ as $j \rightarrow \infty$. Hence $k^{\prime}(f a, f b) \leq \varphi(k(a, b))$.

4.8. Connection with classical quasiconformality. Suppose that $G$ and $G^{\prime}$ are domains in $R^{n}$ and that $f: G \rightarrow G^{\prime}$ is a homeomorphism. Then the conditions " $f$ is $K$-quasiconformal" and " $f$ is freely $\varphi$-quasiconformal" are quantitatively equivalent, provided that the dimension $n$ is included in the data. More precisely, if $f$ is $K$-quasiconformal, then $f$ is $\varphi$-FQC with $\varphi=\varphi_{K, n}$ by [GO, Th. 3]. Conversely, if $f$ is $\varphi$-FQC, then $f$ is $K$-quasiconformal with $K=K(\varphi, n)$. This was proved in [ $\left.\mathrm{TV}_{2}, 6.12\right]$, but essentially the same result was given by F. W. Gehring [Ge, Th. 4] already in 1963. In fact, it is true with $K=K(\varphi)$ by Theorem 7.13 of the present article.

To emphasize the dependence on the dimension $n$, we say that the conditions are $n$-quantitatively equivalent.

4.9. Problems. 1. Is the Gehring-Osgood result in 4.8 true with $\varphi$ depending on $K$ but not on $n$ ? Remember that we are using the simplified metric definition of $K$ quasiconformality; see 1.1. A negative answer would imply that free $\varphi$-quasiconformality cannot be characterized in terms of the metric dilatation $H(x, f)$. If the answer is yes, one can ask:

2. Suppose that $f: G \rightarrow G^{\prime}$ is a homeomorphism between domains in Banach spaces and that $H(x, f) \leq K$ for all $x \in G$. Is $f \varphi$-FQC with $\varphi=\varphi_{K}$ ?

4.10. REMARK. It might look natural to consider also fully $M$-QH and fully $C$-coarsely $M$-QH maps. However, this would not give any new classes. In fact, we shall later show that the $M-\mathrm{QH}$ maps are fully $4 M^{2}$-QH and that the fully $C$-coarsely $M$-QH maps are $\varphi$-FQC with $\varphi=\varphi_{M, C}$.

4.11. Notes. Solid maps in $R^{n}$ were introduced in [TV 2$]$. In the special case where $G=$ $G^{\prime}$ is a half space of $R^{n}$, these maps were considered already in 1964 by V. A. Efremovich and E. S. Tihomirova [ET]. They used the term equimorphism and proved that such a map extends to a homeomorphism $\bar{G} \rightarrow \bar{G}^{\prime}$. We shall prove the corresponding result for coarsely QH maps between uniform domains in Banach spaces in Section 11.

In $R^{n}$, the QH maps form an important subclass of the quasiconformal maps. In fact, since the QH metric and the norm metric are infinitesimally multiples of each other, an $M$ QH map between domains in $R^{n}$ is $M^{2}$-quasiconformal. The Beurling-Ahlfors extension $[\mathrm{BA}]$ and several other extensions are examples of $\mathrm{QH}$ maps.

\section{Quasihyperbolic maps}

5.1. Summary. We show that QH maps have two pleasant properties compared with the other classes introduced in Section 4: (1) There is a local characterization for QH maps. (2) An $M$-QH map is fully $M^{\prime}-\mathrm{QH}$ with $M^{\prime}=4 M^{2}$. We also show that a locally $M$ bilipschitz homeomorphism is $M^{2}-\mathrm{QH}$. Thanks to these properties, it is usually relatively easy to find out whether a given map is $\mathrm{QH}$ or not. The corresponding problem for the other classes is essentially harder. Some examples of $\mathrm{QH}$ maps are given. We also characterize the $\mathrm{QH}$ maps as homeomorphic quasisimilarities. 
5.2. Stretchings. Let $g: X \rightarrow Y$ be a map between metric spaces and let $x$ be a nonisolated point of $x$. We write

$$
L(x, g)=\limsup _{y \rightarrow x} \frac{|f y-f x|}{|y-x|}, \quad l(x, f)=\liminf _{y \rightarrow x} \frac{|f y-f x|}{|y-x|} .
$$

Then $0 \leq l(x, f) \leq L(x, f) \leq \infty$. The number $L(x, f)$ is the maximal stretching of $f$ at $x$, and $l(x, f)$ is the minimal stretching. In the case where $X=G$ and $Y=G^{\prime}$ with the QH metrics, we use the notation $L_{k}(x, f), l_{k}(x, f)$. In 5.4 we express these numbers in terms of the stretchings $L(x, f), l(x, f)$ in the norm metric. First we give some simple general properties of the stretchings.

5.3. Lemma. (1) If $f: X \rightarrow Y$ is a homeomorphism and if $x$ is a nonisolated point of $x$, then

$$
L(x, f)=1 / l\left(f x, f^{-1}\right), l(x, f)=1 / L\left(f x, f^{-1}\right),
$$

where we understand $1 / 0=\infty, 1 / \infty=0$.

(2) Suppose that $f: X \rightarrow Y$ and $g: Y \rightarrow Z$ are continuous and that $x$ and $f x$ are nonisolated points of $X$ and $Y$, respectively. Then

$$
L(x, g \circ f) \leq L(x, f) L(f x, g), l(x, g \circ f) \geq l(x, f) l(f x, g),
$$

provided that the products are not of the form $0 \cdot \infty$ or $\infty \cdot 0$.

(3) Suppose that $G$ is a domain in $E$, that $f$ and $g$ are maps of $G$ into Banach spaces and that $\lambda \in \mathbf{R}$. Then the formulas

$$
\begin{aligned}
& L(x, f \pm g) \leq L(x, f)+L(x, g), \\
& L(x, \lambda f) \leq|\lambda| L(x, f), \\
& L(x, f g) \leq L(x, f)|g x|+|f x| L(x, g)
\end{aligned}
$$

are valid whenever they make sense and contain no product of the form $0 \cdot \infty$ or $\infty \cdot 0$. In the last formula, one of the functions $f, g$ must be real-valued.

Proof. Part (1) is obvious. To prove (2), suppose that $y \in X, y \neq x$ and $f y \neq f x$. Then

$$
\frac{|g f y-g f x|}{|y-x|}=\frac{|g f y-g f x|}{|f y-f x|} \frac{|f y-f x|}{|y-x|} .
$$

Since $f$ is continuous, $f y \rightarrow f x$ as $y \rightarrow x$, and (2) follows easily. The proof of (3) is left as an exercise.

5.4. Theorem. If $f: G \rightarrow G^{\prime}$ is continuous, then

$$
L_{k}(x, f)=\frac{L(x, f) \delta(x)}{\delta^{\prime}(f x)}, \quad l_{k}(x, f)=\frac{l(x, f) \delta(x)}{\delta^{\prime}(f x)} \quad \text { for all } x \in G .
$$

Proof. Let $d$ and $d^{\prime}$ denote the norm metrics of $G$ and $G^{\prime}$, respectively, and let $i$ : $(G, k) \rightarrow(G, d)$ and $j:\left(G^{\prime}, k^{\prime}\right) \rightarrow\left(G^{\prime}, d^{\prime}\right)$ be the identity maps. From parts (3) and (4) of 3.7 it follows that $L(x, i)=\delta(x)$ and $L\left(x, i^{-1}\right)=\delta(x)^{-1}$, and the corresponding formulas hold for $j$. Consider $f$ as a map $f:(G, d) \rightarrow\left(G^{\prime}, d^{\prime}\right)$, and let $f_{k}:(G, k) \rightarrow\left(G^{\prime}, k^{\prime}\right)$ be the 
map with the same values. Since $f_{k}=j^{-1} f i$ and $f=j f_{k} i^{-1}$, Lemma 5.3 gives

$$
\begin{aligned}
& L_{k}(x, f)=L\left(x, f_{k}\right) \leq L(x, i) L(x, f) L\left(f x, j^{-1}\right)=\delta(x) L(x, f) \delta^{\prime}(f x)^{-1}, \\
& L(x, f) \leq L\left(x, i^{-1}\right) L\left(x, f_{k}\right) L(f x, j)=\delta(x)^{-1} L_{k}(x, f) \delta^{\prime}(f x),
\end{aligned}
$$

and we obtain the first formula of the theorem. The proof for the second formula is similar.

5.5. Lemma. Suppose that $X$ is c-quasiconvex and that $f: X \rightarrow Y$ is a map with $L(x, f) \leq M$ for all $x \in X$. Then $f$ is cM-Lipschitz.

Proof. We first consider the case where $X$ is an interval $[a, b]$. If $|f a-f b|>M|a-b|$, an easy bisection argument gives a contradiction. This result is also in [Fe, p. 64].

In the general case, let $a, b \in X$. Join $a$ to $b$ by an $\operatorname{arc} \gamma$ with $\lambda=l(\gamma) \leq c|a-b|$. For the arclength parametrization $\gamma^{0}:[0, \lambda] \rightarrow \gamma$ we have $L\left(t, \gamma^{0}\right) \leq 1$ for all $t \in[0, \lambda]$. By 5.3 this implies that $L\left(t, f \gamma^{0}\right) \leq M$ for all $t$. Hence the special case gives $|f a-f b| \leq$ $M \lambda \leq M c|a-b|$.

5.6. TheOREM. Suppose that $f: G \rightarrow G^{\prime}$ is a homeomorphism with $G \neq E, G^{\prime} \neq E^{\prime}$. Then $f$ is $M-\mathrm{QH}$ if and only if

$$
L(x, f) \delta(x) \leq M \delta^{\prime}(f x), \quad L\left(f x, f^{-1}\right) \delta^{\prime}(f x) \leq M \delta(x) \quad \text { for all } x \in G .
$$

Proof. By 3.4(2), the spaces $(G, k)$ and $\left(G^{\prime}, k^{\prime}\right)$ are $c$-quasiconvex for all $c>1$. The first inequality is equivalent to $L_{k}(x, f) \leq M$ by 5.4 . Hence, by 5.5 , it is equivalent to the condition that $f$ is $M$-Lipschitz in the QH metric. The same is true with $f^{-1}$ and the second inequality.

5.7. Remark. Since $L\left(f x, f^{-1}\right)=l(x, f)^{-1}$, the second inequality of 5.6 can also be written as

$$
\delta^{\prime}(f x) \leq M l(x, f) \delta(x)
$$

5.8. Inversion. As an application of 5.6 we consider the inversion $u: E \backslash\{0\} \rightarrow E \backslash\{0\}$, defined by

$$
u x=\frac{x}{|x|^{2}} .
$$

Observe that $u$ is a homeomorphism with $u^{-1}=u$ and that $|u x|=1 /|x|$. For all $x, y \in$ $E \backslash\{0\}$ we have

$$
\begin{aligned}
|x||y||u x-u y| & =\left|\frac{|y|}{|x|} x-\frac{|x|}{|y|} y\right| \leq\left|\frac{|y|}{|x|} x-x\right|+|x-y|+\left|y-\frac{|x|}{|y|} y\right| \\
& \leq 2|| y|-| x||+|x-y| \leq 3|x-y| .
\end{aligned}
$$

Since $u^{-1}=u$, this gives the double inequality

$$
\frac{1}{3} \frac{|x-y|}{|x||y|} \leq|u x-u y| \leq 3 \frac{|x-y|}{|x||y|}
$$

The constant 3 is the best possible. This is seen by considering $E=R^{2}$ with the norm $|x|=\left|x_{1}\right| \vee\left|x_{2}\right|$ and $x=(1-\varepsilon, 1-\varepsilon), y=(1,1-2 \varepsilon)$ for a small $\varepsilon$. If $E$ is a Hilbert 
space, we can replace (5.9) by the formula

$$
|u x-u y|=\frac{|x-y|}{|x||y|},
$$

which can be verified by direct computation. These formulas imply that $L(x, u) \leq 3 /|x|$ in the general case and $L(x, u)=1 /|x|$ in a Hilbert space. Since $\delta(x)=\delta^{\prime}(x)=|x|$, Theorem 5.6 yields the following result:

5.11. TheOrem. The inversion $u: E \backslash\{0\} \rightarrow E \backslash\{0\}$ is 3-QH. If $E$ is a Hilbert space, then $u$ is 1-QH, that is, a $\mathrm{QH}$ isometry.

5.12. THEOREM. If $f: G \rightarrow G^{\prime}$ is $M-\mathrm{QH}$, then $f$ is fully $4 M^{2}-\mathrm{QH}$.

Proof. Let $D \subset G$ be a domain. Fix a point $x \in D$. By symmetry and by 5.6 , it suffices to show that $L(x, f) \delta_{D}(x) \leq 4 M^{2} \delta_{f D}(f x)$. By auxiliary similarities we normalize the situation so that $\delta_{G}(x)=1=\delta_{G^{\prime}}(f x)$. Since $f$ is $M$-QH, Theorem 5.6 gives $L(x, f) \leq$ $M$. Hence it suffices to show that $\alpha \leq 4 M$ for $\alpha=\delta_{D}(x) / \delta_{f D}(f x)$.

If $\delta_{f D}(f x) \geq 1 / 2 M$, then $\alpha \leq 2 M$. Suppose that $\delta_{f D}(f x)<1 / 2 M$. Choose $0<\varepsilon<$ $1 / 2 M-\delta_{f D}(f x)$ and then a point $y^{\prime} \in \partial f D$ such that

$$
\left|y^{\prime}-f x\right|<\delta_{f D}(f x)+\varepsilon<1 / 2 M .
$$

Then $y^{\prime} \in G^{\prime}$, since

$$
\delta_{G^{\prime}}\left(y^{\prime}\right) \geq \delta_{G^{\prime}}(f x)-\left|y^{\prime}-f x\right|>1-1 / 2 M>0 .
$$

Setting $y=f^{-1}\left(y^{\prime}\right)$ we have $y \in \partial D$. Since

$$
\left|f x-y^{\prime}\right|<1 / 2 M \leq 1 / 2=\delta_{G^{\prime}}(f x) / 2,
$$

Lemma 3.9 gives

$$
k^{\prime}\left(f x, y^{\prime}\right) \leq \frac{2\left|f x-y^{\prime}\right|}{\delta_{G^{\prime}}(f x)}=2\left|f x-y^{\prime}\right|<1 / M
$$

Since $f$ is $M$-QH, this implies $k(x, y)<1$. Applying again 3.9 and the $M$-QH property we get

$$
\begin{aligned}
\delta_{D}(x) & \leq|x-y| \leq 2 k(x, y) \delta_{G}(x)=2 k(x, y) \leq 2 M k^{\prime}\left(f x, y^{\prime}\right) \\
& \leq 4 M\left|f x-y^{\prime}\right|<4 M\left(\delta_{f D}(f x)+\varepsilon\right) .
\end{aligned}
$$

Hence $\alpha<4 M\left(\delta_{f D}(f x)+\varepsilon\right) / \delta_{f D}(x)$. As $\varepsilon \rightarrow 0$, this gives $\alpha \leq 4 M$.

5.13. Corollary. An $M$-QH map is $\varphi$-FQC with $\varphi(t)=4 M^{2} t$.

5.14. REMARK. From 5.11 and 5.12 it follows that the inversion $u: E \backslash\{0\} \rightarrow E \backslash\{0\}$ is fully $36-\mathrm{QH}$. In a Hilbert space it is fully 4-QH.

5.15. Locally bilipschitz maps. A map $f: X \rightarrow Y$ is locally $M$-bilipschitz, $M \geq 1$, if each point $x \in X$ has a neighborhood $U$ such that the restriction $f \mid U$ is $M$-bilipschitz. If $f: G \rightarrow G^{\prime}$ is a homeomorphism between domains in $R^{n}$ and if $f$ is locally $M$-bilipschitz, it follows at once from the definition in 1.1 that $f$ is $M^{2}$-quasiconformal. We next show that such a map is $\mathrm{QH}$ in all Banach spaces.

5.16. TheOREM. Suppose that $f: G \rightarrow G^{\prime}$ is a locally $M$-bilipschitz homeomorphism with $G \neq E$. Then $G^{\prime} \neq E^{\prime}$ and $f$ is $M^{2}-\mathrm{QH}$. 
Proof. We first show that $G^{\prime} \neq E^{\prime}$. Fix a point $a \in G$. Since $G \neq E$, there is a point $b_{1} \in \partial G$. Since $\left[a, b_{1}\right] \cap \partial G$ is compact, there is $b \in\left[a, b_{1}\right]$ such that $\alpha=[a, b) \subset G$. Since $f$ is locally $M$-bilipschitz, we have $L(x, f) \leq M$ for all $x \in G$. Since $\alpha$ is convex, $f \mid \alpha$ is $M$-Lipschitz by 5.5 . Let $\left(x_{j}\right)$ be a sequence on $\alpha$ converging to $b$. Then $\left(f x_{j}\right)$ is a Cauchy sequence and hence converges to a point $y \in E^{\prime}$. This point lies in $E^{\prime} \backslash G^{\prime}$, since otherwise $x_{j} \rightarrow f^{-1}(y)$. Thus $G^{\prime} \neq E^{\prime}$.

Fix a point $x \in G$. By symmetry and by 5.6 , it suffices to show that $\delta(x) \leq M \delta^{\prime}(f x)$. Let $\lambda>1$ and choose a point $y^{\prime} \in \partial G^{\prime}$ such that $\left|y^{\prime}-f x\right| \leq \lambda \delta^{\prime}(f x)$. As above, we may assume that $\beta=\left[f x, y^{\prime}\right) \subset G^{\prime}$. Then $f^{-1} \mid \beta$ is $M$-Lipschitz. Arguing as above we see that $f^{-1}(z)$ has a limit $y \in E$ as $z \rightarrow y^{\prime}$ on $\beta$. We have $y \notin G$, since otherwise $y^{\prime}=f y \in G^{\prime}$. Hence

$$
\delta(x) \leq|x-y| \leq M\left|f x-y^{\prime}\right| \leq M \lambda \delta^{\prime}(f x) .
$$

As $\lambda \rightarrow 1$, this gives $\delta(x) \leq M \delta^{\prime}(f x)$ as desired.

5.17. Remark. The proof of 5.16 is the first time in this article when we actually need the completeness of the spaces $E$ and $E^{\prime}$. The theory before 5.16 makes sense in all normed vector spaces. However, Theorem 5.16 breaks down in a drastic way if $E$ is noncomplete. In fact, let $E$ be a noncomplete normed space. For every $M>1$ it is possible to construct an $M$-bilipschitz homeomorphism $g: E \backslash\{0\} \rightarrow E$ such that $g x=x$ for $|x| \geq 1$. See $\left[V \ddot{a}_{8}\right]$ and the earlier work of C. Bessaga ([Bes], [BP, Prop. 5.1, p. 106]). This $g$ defines a homeomorphism $f$ of $G=B(2) \backslash\{0\}$ onto $G^{\prime}=B(2)$, which is not QH or even solid. To see this, let $a \in G$ with $|a|=r<1 / 2$. Then $k(a, 2 a) \geq \log 2$ by $3.7(1)$. On the other hand, $3.7(3)$ gives

$$
k^{\prime}(f(a), f(2 a)) \leq|f(a)-f(2 a)| \leq M r \rightarrow 0
$$

as $r \rightarrow 0$. Hence $f^{-1}$ cannot be uniformly continuous in the $\mathrm{QH}$ metric.

It seems to the author that there is no useful theory of quasiconformality in general normed spaces.

5.18. Differentiable maps. A map $f: G \rightarrow E^{\prime}$ is differentiable or Fréchet differentiable at a point $x \in G$ if there is a continuous (= bounded) linear map $f^{\prime}(x): E \rightarrow E^{\prime}$, called the derivative of $f$ at $x$, such that

$$
f(x+h)=f(x)+f^{\prime}(x) h+|h| \varepsilon(x, h),
$$

where $\varepsilon(x, h) \rightarrow 0$ as $h \rightarrow 0$. At such a point $x$ we have

$$
L(x, f)=\left|f^{\prime}(x)\right|=\sup \left\{\left|f^{\prime}(x) h\right|:|h|=1\right\} .
$$

For example, if $E$ is a Hilbert space, the norm function $N: E \rightarrow \mathbf{R}, N x=|x|$, is differentiable for $x \neq 0$ with

$$
N^{\prime}(x) h=(x \cdot h) /|x|
$$

Here exceptionally $\operatorname{dim} E^{\prime}=1$. 
If $E=R^{n}, E^{\prime}=R^{p}$, and we write $f=\left(f_{1}, \ldots, f_{p}\right)$, then the elements of the matrix of $f^{\prime}(x)$ are the partial derivatives $\partial_{i} f_{j}(x)=e_{j} \cdot f^{\prime}(x) e_{i}$. The elementary estimate

$$
\left|f^{\prime}(x)\right|^{2} \leq \sum_{i=1}^{n} \sum_{j=1}^{p} \partial_{i} f_{j}(x)^{2}
$$

is often useful when estimating $L(x, f)$.

5.21. The radial power map. Let $E$ be a Banach space. For $\alpha>0$ we define the radial power map $f_{\alpha}: E \rightarrow E$ by $f_{\alpha} x=|x|^{\alpha-1} x$. Then $f_{\alpha}$ is a homeomorphism onto $E$ with $f_{\alpha}^{-1}=f_{1 / \alpha}$. Let $G=G^{\prime}=E \backslash\{0\}$, and let $f: G \rightarrow G^{\prime}$ be the homeomorphism defined by $f_{\alpha}$. Assuming that $\alpha \geq 1$, it is not difficult to show that $L(x, f)=\alpha|x|^{\alpha-1}$ and $L\left(f x, f^{-1}\right) \leq \alpha /|x|^{\alpha-1}$. If $E$ is a Hilbert space, this can be seen from the derivative

$$
f^{\prime}(x) h=(\alpha-1)|x|^{\alpha-2}(x \cdot h) x+|x|^{\alpha-1} h,
$$

obtained by using ordinary differentiation rules and (5.19). For general Banach spaces, see [I, 4.10]. Since $\delta(x)=|x|$ and $\delta^{\prime}(f x)=|f x|=|x|^{\alpha}$, we conclude by 5.6 that $f$ is $\alpha-\mathrm{QH}$

5.22. Radial bilipschitz maps. We shall later make use of the following kind of maps. Let $r_{1}, r_{2} \in(0, \infty]$ and let $g:\left[0, r_{1}\right) \rightarrow\left[0, r_{2}\right)$ be an $M$-bilipschitz homeomorphism. For $x \in B\left(r_{1}\right) \backslash\{0\}$ we set

$$
p x=x /|x|, f x=g(|x|) p x .
$$

For all $x, y \in B\left(r_{1}\right) \backslash\{0\}$ we have

$$
\begin{aligned}
|f x-f y| & \leq g(|x|)|p x-p y|+|g(|x|)-g(|y|)||p y| \\
& \leq M|x||p x-p y|+M|x-y| .
\end{aligned}
$$

Since

$$
|x||p x-p y|=\left|x-\frac{|x| y}{|y|}\right| \leq|x-y|+|| y|-| x|| \leq 2|x-y|,
$$

we see that $f$ is $3 M$-Lipschitz. Extending $f$ to $B\left(r_{1}\right)$ by $f(0)=0$ and observing that $f^{-1}(x)=g^{-1}(|x|) p x$, we obtain a $3 M$-bilipschitz homeomorphism $f: B\left(r_{1}\right) \rightarrow B\left(r_{2}\right)$. The case $r_{1}=r_{2}=\infty$ is allowed; then $f: E \rightarrow E$.

5.23. Quasisimilarities. Suppose that $f: G \rightarrow G^{\prime}$ is a homeomorphism with $G \neq$ $E, G^{\prime} \neq E^{\prime}$. Let $0<q<1$ and $M \geq 1$. We say that $f$ is a homeomorphic $(M, q)$ quasisimilarity if

$$
L(x, f) \leq M l(y, f), L(y, f) \leq M l(x, f)
$$

whenever $x, y \in G$ and $|y-x|<q \delta(x)$.

Observe that the numbers in (5.24) are not zero or infinite. Indeed, if $l(x, f)=0$ for some $x$, then $L(y, f)=0$ for $|y-x|<q \delta(x)$, and hence $f \mid B(x, q \delta(x))$ is constant by 5.5 . If $L(x, f)=\infty$ for some $x$, then $l(y, f)=\infty$ for $|y-x|<q \delta(x)$. Hence $L\left(z, f^{-1}\right)=0$ in a ball neighborhood $V$ of $f x$, and thus $f^{-1} \mid V$ is constant.

We remark that in $R^{n}$ one can consider also nonhomeomorphic quasisimilarities. The condition that $f$ is sense-preserving is then added. 
We first show that the inequalities in (5.24) are quantitatively equivalent to each other. Then we prove that $f$ is $\mathrm{QH}$ if and only if $f$ and $f^{-1}$ are homeomorphic quasisimilarities.

5.25. LEMmA. If a homeomorphism $f: G \rightarrow G^{\prime}$ satisfies one of the inequalities of (5.24), it satisfies also the other with the same $q$ and with $M^{\prime}=M^{\prime}(M, q)$.

ProOf. Suppose that the first inequality of (5.24) is true, and let $x, y \in G$ with $|x-y|<q \delta(x)$. Set $t=q(1-q) \delta(x)$, and let $m \geq 1$ be the unique integer with $(m-$ $1) t=|y-x|<m t$. Divide the segment $[y, x]$ into $m$ equal parts by successive points $y=y_{0}, \ldots, y_{m}=x$. Then $\left|y_{j-1}-y_{j}\right|=|y-x| / m<t$ for all $1 \leq j \leq m$. Furthermore,

$$
\delta\left(y_{j-1}\right) \geq \delta(x)-\left|x-y_{j-1}\right|>(1-q) \delta(x)=t / q .
$$

It follows that

$$
L\left(y_{j-1}, f\right) \leq M l\left(y_{j}, f\right) \leq M L\left(y_{j}, f\right)
$$

for $1 \leq j \leq m$. Hence $L(y, f) \leq M^{m} l(x, f)$. Since $m-1 \leq|y-x| / t<1 /(1-q)$, we have $m<2 /(1-q)$. Consequently, the second inequality if $(5.24)$ is true with $M$ replaced by $M^{\prime}=M^{2 /(1-q)}$.

The proof for the converse is similar.

5.26. Theorem. Suppose that $f: G \rightarrow G^{\prime}$ is a homeomorphism with $G \neq E, G^{\prime} \neq E^{\prime}$. Then the following conditions are quantitatively equivalent:

(1) $f$ is $M-\mathrm{QH}$.

(2) For each $0<q<1$ there is $M \geq 1$ such that $f$ and $f^{-1}$ are homeomorphic $(M, q)$-quasisimilarities.

(3) $f$ and $f^{-1}$ are $(M, q)$-quasisimilarities.

Proof. We show that $(1) \Rightarrow(2) \Rightarrow(3) \Rightarrow(1)$. The implication (2) $\Rightarrow(3)$ is trivial. We next show that (1) implies (2) quantitatively. Let $0<q<1$ and let $x, y \in G$ with $|x-y|<q \delta(x)$. Since $f$ is $M$-QH, Theorem 5.6 implies that

$$
L(x, f) \delta(x) \leq M \delta^{\prime}(f x), \delta^{\prime}(f y) \leq M \delta(y) l(y, f) .
$$

By 5.25, it suffices to prove the first inequality of (5.24). In view of (5.27), it suffices to find $M^{\prime}=M^{\prime}(M, q)$ such that

$$
\delta^{\prime}(f x) \delta(y) \leq M^{\prime} \delta^{\prime}(f y) \delta(x) .
$$

Since $f$ is $M$-QH, parts (1) and (5) of 3.7 yield

$$
\log \frac{\delta^{\prime}(f y)}{\delta^{\prime}(f x)} \leq k^{\prime}(f x, f y) \leq M k(x, y) \leq M \log \frac{1}{1-q}
$$

Moreover, $\delta(y) \leq(1+q) \delta(x)<\delta(x) /(1-q)$. Hence (5.28) holds with $M^{\prime}=(1-q)^{-M-1}$.

It remains to show that $(3) \Rightarrow(1)$. Assume that (3) holds. We show that $f$ is $(M / q)$ QH. Let $x \in G$ and write $t=L(x, f) q \delta(x) / M$. By symmetry and by 5.6 , it suffices to show that $\delta^{\prime}(f x) \geq t$. This will follow if $|f x-f y| \geq t$ for each $y$ such that $|y-x|=q \delta(x)$ and $[f x, f y) \subset f B(x, q \delta(x))$. For every $z \in[f x, f y)$ we have

$$
L\left(z, f^{-1}\right)=1 / l\left(f^{-1}(z), f\right) \leq M / L(x, f) .
$$


By 5.5 this implies that

$$
q \delta(x)=|x-y| \leq \frac{M|f x-f y|}{L(x, f)}
$$

and hence $|f x-f y| \geq t$ as desired.

5.29. Notes. As noted in 4.11, $M$-QH maps in $R^{n}$ are $M^{2}$-quasiconformal. If $n \neq 4$, every quasiconformal map between proper subdomains of $R^{n}$ can be approximated by $\mathrm{QH}$ maps, uniformly in the $\mathrm{QH}$ metric $\left[\mathrm{TV}_{2}, 7.12\right]$.

Homeomorphic quasisimilarities between domains in $R^{2}$ were introduced by K. Astala and F. W. Gehring [AG] in 1986. The notion was generalized to $R^{n}$ and for nonhomeomorphic maps by O. Martio [Ma]. The equivalence 5.26 of homeomorphic quasisimilarities and QH maps in $R^{n}$ was proved by K. Hag, P. Hag and O. Martio [HHM, 2.40] and also by T. G. Latfullin [Lat, Th. 1]. However, the condition that $f^{-1}$ is an $(M, q)$-quasisimilarity can be dropped in $R^{n}$, because it follows $n$-quantitatively from the $(M, q)$-quasisimilarity property of $f$; see [HHM, 2.33]. I do not know whether the corresponding free statement is true.

\section{Quasisymmetric and quasimöbius maps}

6.1. Summary. Quasisymmetry is a global version of quasiconformality, and it can be considered in arbitrary metric spaces. In Section 7 we shall characterize the FQC maps in terms of local quasisymmetry. This section gives the basic theory of quasisymmetry in metric spaces. We also consider the more general class of quasimöbius maps.

6.2. Quasisymmetry. Let $\eta:[0, \infty) \rightarrow[0, \infty)$ be a homeomorphism. A map $f: X \rightarrow Y$ between metric spaces is $\eta$-quasisymmetric if

(1) $f$ is an embedding,

(2) $|a-x| \leq t|b-x|$ implies $|f a-f x| \leq \eta(t)|f b-f x|$ for all $x, a, b \in X$ and $t \geq 0$.

The condition (2) can also be written as

$$
\frac{|f a-f x|}{|f b-f x|} \leq \eta\left(\frac{|a-x|}{|b-x|}\right)
$$

for each triple $x, a, b$ of distinct points in $X$. The condition (1) can be replaced by (1') $f$ is injective or even by (1") $f$ is nonconstant by the following result:

6.3. TheOrem. Suppose that $f: X \rightarrow Y$ is a function satisfying condition (2) of 6.2. Then $f$ is either a constant or an $\eta$-quasisymmetric embedding. In the latter case, $f^{-1}: f X \rightarrow X$ is $\eta^{\prime}$-quasisymmetric with $\eta^{\prime}(t)=\eta^{-1}\left(t^{-1}\right)^{-1}$.

Proof. Suppose that $f$ is not constant. We first show that $f$ is injective. Assume that $f x=f y$ for some $x \neq y$. Let $z \in X$ and write $|z-x|=t|y-x|$. Then $|f z-f x| \leq$ $\eta(t)|f y-f x|=0$. Hence $f$ is constant, a contradiction.

We next show that $f$ is continuous at an arbitrary point $x_{0} \in X$. Fix a point $b \in X \backslash$ $\left\{x_{0}\right\}$, let $\varepsilon>0$, and choose $t>0$ with $\eta(t)\left|f b-f x_{0}\right|<\varepsilon$. If $x \in X$ and $\left|x-x_{0}\right| \leq t\left|b-x_{0}\right|$, then $\left|f x-f x_{0}\right|<\varepsilon$, and hence $f$ is continuous at $x_{0}$. 
Suppose that $x, a, b \in X$ and $t>0$ are such that $|f a-f x| \leq t|f b-f x|$. If $|a-x|>$ $\eta^{\prime}(t)|b-x|$, then

$$
|f b-f x|<\eta\left(\eta^{\prime}(t)^{-1}\right)|f a-f x|=t^{-1}|f a-f x|,
$$

a contradiction. In view of what was proved above, this completes the proof.

6.4. Weaker conditions. 1 . Note that in the definition 6.2 of quasisymmetry we have $\eta(0)=0$. It is sufficient that $6.2(2)$ holds with some function $\eta$ such that $\eta$ is bounded on bounded intervals and $\eta(t) \rightarrow 0$ as $t \rightarrow 0$; cf. 2.2. If it holds with some increasing function $\eta$ with $\eta(0)>0$, the map need not be quasisymmetric. However, if $f$ and $f^{-1}: f X \rightarrow X$ satisfy this condition, then $f$ is $\eta_{1}$-quasisymmetric with $\eta_{1}$ depending only on $\eta$. This follows from the corresponding relative theorem 6.27 with $A=X$.

2. A still weaker condition is the following. Let $H \geq 1$. An embedding $f: X \rightarrow Y$ is said to be weakly $H$-quasisymmetric if $|a-x| \leq|b-x|$ implies $|f a-f x| \leq H|f b-f x|$ for all $x, a, b \in X$. If $f$ satisfies the condition of 6.4 .1 above, then $f$ is trivially weakly $H$-quasisymmetric with $H=\eta(1)$. In particular, an $\eta$-quasisymmetric map is weakly $\eta(1)$-quasisymmetric. The converse is not true; a counterexample is given in 8.5. Weak quasisymmetry is not important as an independent concept but it is useful, since (1) it is more easily verified than quasisymmetry, and (2) in certain situations it is equivalent to quasisymmetry. We give an example of (2) in 6.6. Its proof is based on the following lemma, which is useful also elsewhere in this article.

6.5. LemmA. Suppose that $X$ is c-quasiconvex, that $f: X \rightarrow Y$ is weakly H-quasisymmetric and that $x, a, b$ are distinct points in $X$ with $|a-x| \leq t|b-x|$. Then

$$
|f a-f x| \leq M|f b-f x|
$$

for some $M=M(t, H, c)$, which is increasing in $t$.

Proof. Observe that the lemma does not say that $f$ is quasisymmetric, since $M(t, H, c)$ need not tend to zero as $t \rightarrow 0$.

If $t \leq 1$, we can choose $M=H$. Assume that $t>1$. By quasiconvexity we can join $x$ to $a$ by an arc $\gamma$ with $l(\gamma) \leq c|a-x|$. Define inductively the successive points $x=x_{0}, \ldots, x_{s}=a$ of $\gamma$ so that $x_{j}$ is the last point of $\gamma$ in $\bar{B}\left(x_{j-1},|x-b|\right)$. The process is finite by the compactness of $\gamma$. Then $s \geq 2,\left|x_{j-1}-x_{j}\right|=|b-x|$ for $1 \leq j \leq s-1$, and $\left|x_{s-1}-x_{s}\right| \leq|b-x|$. Since $f$ is weakly $H$-quasisymmetric, we have

$$
\begin{aligned}
& \left|f x_{1}-f x_{0}\right| \leq H|f b-f x|, \\
& \left|f x_{2}-f x_{1}\right| \leq H\left|f x_{1}-f x_{0}\right| \leq H^{2}|f b-f x|, \\
& \cdots \\
& \left|f x_{s}-f x\right| \leq H^{s}|f b-f x| .
\end{aligned}
$$

Summation gives

$$
|f a-f x| \leq\left(H+H^{2}+\ldots+H^{s}\right)|f b-f x| \leq s H^{s}|f b-f x| .
$$

Since

$$
l\left(\alpha\left[x_{j-1}, x_{j}\right]\right) \geq\left|x_{j-1}-x_{j}\right|=|b-x|
$$


for $1 \leq j \leq s-1$, we have

$$
(s-1)|b-x| \leq l(\gamma) \leq c|a-x| \leq c t|b-x| .
$$

Hence $s \leq 1+c t$, and we obtain the lemma with $M=(1+c t) H^{1+c t}$.

6.6. TheOREM. Suppose that $f: X \rightarrow Y$ is weakly $H$-quasisymmetric and that $X$ and $f X$ are $c$-quasiconvex. Then $f$ is $\eta$-quasisymmetric with $\eta$ depending only on $H$ and $c$.

Proof. Let $x, a, b$ be distinct points in $X$ and set $t=|a-x| /|b-x|, t^{\prime}=\mid f a-$ $f x|/| f b-f x \mid$. We want to find an estimate $t^{\prime} \leq \eta(t)$ where $\eta(t) \rightarrow 0$ as $t \rightarrow 0$. Since 6.5 gives $t^{\prime} \leq M(t, H, c)$ for all $t$, we may assume that $0<t \leq 1 / 4$.

Since $f X$ is $c$-quasiconvex, we can join $f x$ and $f b$ by an arc $\gamma \subset f X$ such that $l(\gamma) \leq$ $c|f b-f x|$. Choose successive points $b=b_{0}, \ldots, b_{s}$ of $f^{-1} \gamma$ such that $\left|b_{j}-x\right|=3^{-j}|b-x|$ and $s$ is the least integer with $3^{-s} \leq t$. Since $t \leq 1 / 4$, we have $s \geq 2$. Moreover

$$
s \geq \frac{\log (1 / t)}{\log 3}=s_{0}(t) \rightarrow \infty \quad \text { as } t \rightarrow 0 .
$$

For $1 \leq j \leq s-1$ we have

$$
\left|b_{j}-a\right| \leq\left|b_{j}-x\right|+|x-a| \leq 2\left|b_{j}-x\right| \leq\left|b_{j}-b_{j-1}\right| .
$$

Moreover, $\left|b_{j}-x\right| \leq\left|b_{j}-b_{j-1}\right| / 2<\left|b_{j}-b_{j-1}\right|$. Since $f$ is weakly $H$-quasisymmetric, we obtain

$$
|f a-f x| \leq\left|f a-f b_{j}\right|+\left|f b_{j}-f x\right| \leq 2 H\left|f b_{j}-f b_{j-1}\right| .
$$

Summing over $1 \leq j \leq s-1$ yields

$$
(s-1)|f a-f x| \leq 2 H l(\gamma) \leq 2 H c|f b-f x| .
$$

Hence we can choose $\eta(t)=2 H c /\left(s_{0}(t)-1\right)$.

6.7. Corollary. A homeomorphism $f: E \rightarrow E^{\prime}$ is $\eta$-quasisymmetric if and only if, quantitatively, $f$ is weakly $H$-quasisymmetric.

6.8. EXAMPLES. 1. An $M$-bilipschitz map $f: X \rightarrow Y$ is clearly $\eta$-quasisymmetric with $\eta(t)=M^{2} t$.

2. Let $E$ be a Banach space, let $\alpha>1$, and let $f: E \rightarrow E$ be the radial power map $f x=|x|^{\alpha-1} x$, considered in 5.21. This map is not bilipschitz, but it is $\eta$-quasisymmetric with some $\eta=\eta_{\alpha}$. A direct proof for this seems to be very awkward although it suffices to prove that $f$ is weakly $H_{\alpha}$-quasisymmetric by 6.7 . We sketch two different proofs.

(a) Let $x, a, b \in E$. We may assume that $\operatorname{dim} E \geq 3$. Choose a 3-dimensional linear subspace $E_{0}$ of $E$ containing these points. Since $f E_{0}=E_{0}$, we see that it suffices to consider the case $\operatorname{dim} E=3$. If $E$ is Hilbert, we may assume that $E=R^{3}$. Then one can show that $f$ is $\alpha$-quasiconformal, and the result follows from the theory of 3 -dimensional quasiconformal maps; see 6.9. The general case reduces to the Hilbert case by means of auxiliary bilipschitz maps; see [I, 6.2].

(b) Set $G=E \backslash\{0\}$ and let $g: G \rightarrow G$ be the homeomorphism defined by $f$. We proved in 5.21 that $g$ is $\alpha$-QH. In Section 10 we shall introduce the uniform domains and show that $G$ is $c_{0}$-uniform with a universal constant $c_{0}$. From Theorem 11.5 it will follow that $g$ is $\eta$-quasisymmetric with $\eta=\eta_{\alpha}$. By continuity, also $f$ is $\eta$-quasisymmetric. 
6.9. Quasisymmetry and quasiconformality in $R^{n}$. Suppose that $G$ and $G^{\prime}$ are domains in $R^{n}$ and that $f: G \rightarrow G^{\prime}$ is a homeomorphism. If $f$ is weakly $H$-quasisymmetric, then clearly $f$ is $H$-quasiconformal according to the definition in 1.1. Conversely, a quasiconformal map need not be weakly quasisymmetric, since the boundary behavior easily destroys quasisymmetry. However, a quasiconformal map is always locally quasisymmetric. In fact, the following result is true [V $\left.\ddot{a}_{3}, 2.4\right]$ :

Let $f: G \rightarrow G^{\prime}$ be a $K$-quasiconformal map between domains $G, G^{\prime} \subset R^{n}$ and let $0<q<1$. Then $f \mid B(x, q \delta(x))$ is $\eta$-quasisymmetric with $\eta$ depending only on $K, q$ and $n$.

In particular, a homeomorphism $f: R^{n} \rightarrow R^{n}$ is $K$-quasiconformal if and only if, $n$-quantitatively, $f$ is $\eta$-quasisymmetric. It is not known whether $\eta$ can be chosen to be independent of $n$.

We say that $f$ is q-locally $\eta$-quasisymmetric if it has the property of the above result. We see that for homeomorphisms between domains in $R^{n}, K$-quasiconformality is $n$ quantitatively equivalent to $q$-local $\eta$-quasisymmetry. In 7.12 we show that in all Banach spaces, free $\varphi$-quasiconformality is quantitatively equivalent to the property that $f$ and $f^{-1}$ are $q$-locally $\eta$-quasisymmetric.

6.10. Properties of quasisymmetric maps. We already observed in 6.3 that the inverse map $f^{-1}: f X \rightarrow X$ of an $\eta$-quasisymmetric map is $\eta^{\prime}$-quasisymmetric with $\eta^{\prime}(t)=$ $\eta^{-1}\left(t^{-1}\right)^{-1}$. If $f: X \rightarrow Y$ is $\eta_{1}$-quasisymmetric and $g: Y \rightarrow Z$ is $\eta_{2}$-quasisymmetric, then clearly $g f: X \rightarrow Z$ is $\eta$-quasisymmetric with $\eta(t)=\eta_{2}\left(\eta_{1}(t)\right)$.

In 6.11-6.13 we give some qualitative properties of quasisymmetric maps. In 6.14 we show that for $\eta$-quasisymmetric maps between connected spaces, the function $\eta$ can always be chosen to be of the form $\eta(t)=C\left(t^{\alpha} \vee t^{1 / \alpha}\right)$. This will be applied in 6.15 to show that quasisymmetric maps of connected spaces are Hölder continuous.

6.11. Theorem. Let $f: X \rightarrow Y$ be quasisymmetric. Then $f$ maps

(1) bounded sets to bounded sets,

(2) Cauchy sequences to Cauchy sequences,

(3) complete sets to complete sets.

Proof. Assume that $f$ is $\eta$-quasisymmetric. Let $A \subset X$ be bounded. Fix points $x, b \in A$ with $x \neq b$. For each $a \in A$ we have $|a-x| \leq(d(A) /|b-x|)|b-x|$, and hence

$$
|f a-f x| \leq \eta\left(\frac{d(A)}{|b-x|}\right)|f b-f x|
$$

which implies that $f A$ is bounded.

Let $\left(x_{j}\right)$ be a Cauchy sequence in $X$. Set $A=\left\{x_{j}: j \in \mathbf{N}\right\}$. Then $f A$ is bounded by (1). We may assume that $d(A)>0$. Let $i, j \in \mathbf{N}$ and choose $s \in \mathbf{N}$ with $\left|x_{s}-x_{j}\right| \geq d(A) / 3$. Then

$$
\left|f x_{i}-f x_{j}\right| \leq \eta\left(\frac{\left|x_{i}-x_{j}\right|}{\left|x_{s}-x_{j}\right|}\right)\left|f x_{s}-f x_{j}\right| \leq \eta\left(\frac{3\left|x_{i}-x_{j}\right|}{d(A)}\right) d(f A),
$$

and (2) follows.

Finally, assume that $A \subset X$ is complete and that $\left(x_{j}\right)$ is a sequence in $A$ such that $\left(f x_{j}\right)$ is a Cauchy sequence. Since $f^{-1}$ is quasisymmetric, $\left(x_{j}\right)$ is a Cauchy sequence by (2), and hence $x_{j} \rightarrow a \in A$. By continuity, $f x_{j} \rightarrow f a \in f A$. 
6.12. TheOrem. Suppose that $X, Y$ are metric spaces, that $A \subset X$, that $f: A \rightarrow Y$ is $\eta$-quasisymmetric, and that $\overline{f A}$ is complete. Then $f$ extends to an $\eta$-quasisymmetric $\operatorname{map} g: \bar{A} \rightarrow Y$.

Proof. By 6.11, the image of every Cauchy sequence of $A$ is convergent. Hence $f$ has a unique extension to a continuous map $g: \bar{A} \rightarrow Y$. Since $\eta$ is continuous, we have $|g a-g x| \leq \eta(t)|g b-g x|$ whenever $x, a, b \in \bar{A}$ and $|a-x| \leq t|b-x|$. Hence $g$ is $\eta$-quasisymmetric by 6.3 .

The following version of 6.11(3) for weakly quasisymmetric maps will be needed in 7.9.

6.13. Lemma. Suppose that $X$ is bounded, complete and quasiconvex and that $f$ : $X \rightarrow Y$ is weakly quasisymmetric. Then $f X$ is complete.

Proof. Suppose that $X$ is $c$-quasiconvex and that $f$ is weakly $H$-quasisymmetric. Let $\left(x_{j}\right)$ be a sequence in $X$ such that $\left(f x_{j}\right)$ is a Cauchy sequence. It suffices to show that $\left(x_{j}\right)$ is a Cauchy sequence, since then it converges to a point $a \in X$ and $f x_{j} \rightarrow f a$. Assume that $\left(x_{j}\right)$ is not Cauchy. Then there is $\varepsilon>0$ such that for each $k \in \mathbf{N}$ there is $j(k)>k$ with $\left|x_{k}-x_{j(k)}\right| \geq \varepsilon$. Let $k \in \mathbf{N}$ and let $z_{k} \in\left\{x_{k}, x_{j(k)}\right\}$ be a point with $\left|z_{k}-x_{1}\right| \geq \varepsilon / 2$. Writing $t=d(X) / \varepsilon$ we have

$$
\left|x_{1}-x_{j(1)}\right| \leq d(X)=t \varepsilon \leq 2 t\left|z_{k}-x_{1}\right| .
$$

By 6.5 this implies that

$$
\left|f x_{1}-f x_{j(1)}\right| \leq M(2 t, H, c)\left|f z_{k}-f x_{1}\right|
$$

for some function M. Similarly, $\left|z_{k}-x_{1}\right| \leq t \varepsilon \leq t\left|x_{k}-x_{j(k)}\right|$ implies

$$
\left|f z_{k}-f x_{1}\right| \leq M(t, H, c)\left|f x_{k}-f x_{j(k)}\right| .
$$

Since $\left(f x_{j}\right)$ is Cauchy, the right-hand side tends to zero as $k \rightarrow \infty$. Hence these inequalities give $\left|f x_{1}-f x_{j(1)}\right|=0$, a contradiction.

6.14. TheOREM. Suppose that $X$ is a connected metric space and that $f: X \rightarrow Y$ is $\eta$-quasisymmetric. Then $f$ is $\eta_{1}$-quasisymmetric with a function $\eta_{1}(t)=C\left(t^{\alpha} \vee t^{1 / \alpha}\right)$, where $C>0$ and $\alpha \in(0,1]$ depend only on $\eta$.

Proof. We may assume that $\eta(t) \geq t$. Setting $H=\eta(2)$ and $h=\eta^{-1}(1 / 2)$ we have $H \geq 2$ and $h \leq 1 / 2$. Let $x, a, b$ be distinct points in $X$, and set $t=|a-x| /|b-x|, t^{\prime}=$ $|f a-f x| /|f b-f x|$. We must find $C$ and $\alpha$ such that

$$
t^{\prime} \leq C\left(t^{\alpha} \vee t^{1 / \alpha}\right)
$$

We consider two cases.

Case 1. $t \geq 1$. Since $X$ is connected, there is a sequence $a=x_{0}, \ldots, x_{m}$ such that $m \geq 0$ and

$$
\left|x_{j}-x\right|=2^{-j}|a-x|, 2^{-m-1}|a-x|<|b-x| \leq 2^{-m}|a-x| .
$$

Writing $x_{m+1}=b$ we have $\left|f x_{j}-f x\right| \leq H\left|f x_{j-1}-f x\right|$ for $1 \leq j \leq m+1$, and hence $t^{\prime} \leq H^{m+1}$. Since $t \geq 2^{m}$, we get

$$
t^{\prime} \leq H^{1 / \alpha}
$$

with $\alpha=\log 2 / \log H \leq 1$. 
Case 2. $t \leq 1$. Choose points $b=y_{0}, \ldots, y_{n}$ such that $n \geq 0$ and

$$
\left|y_{j}-x\right|=h^{j}|b-x|, h^{n+1}|b-x|<|a-x| \leq h^{n}|b-x| .
$$

Then $\left|f y_{j}-f x\right| \leq\left|f y_{j-1}-f x\right| / 2$ for $1 \leq j \leq n$, and hence $\left|f y_{n}-f x\right| \leq 2^{-n}|f b-f x|$. Since $|f a-f x| \leq H\left|f y_{n}-f x\right|$, we have $t^{\prime} \leq 2^{-n} H$. Since $t \geq h^{n+1}$, this gives

$$
t^{\prime} \leq 2 H t^{\alpha}
$$

with $\alpha=\log 2 / \log (1 / h) \leq 1$.

6.15. TheOREM. Suppose that $X$ is connected, that $f: X \rightarrow Y$ is $\eta$-quasisymmetric and that $A \subset X$ is bounded. Then $f \mid A$ satisfies a two-sided Hölder condition

$$
|x-y|^{1 / \alpha} / M \leq|f x-f y| \leq M|x-y|^{\alpha} \quad \text { for } x, y \in A \text {, }
$$

where $\alpha=\alpha(\eta) \leq 1$ and $M=M(\eta, d(A), d(f A)) \geq 1$.

Proof. Since $f^{-1}: f X \rightarrow X$ is $\eta^{\prime}$-quasisymmetric with $\eta^{\prime}(t)=\eta^{-1}\left(t^{-1}\right)^{-1}$, it suffices to prove the second inequality. By 6.14 we may assume that $\eta(t)=C\left(t^{\alpha} \vee t^{1 / \alpha}\right)$ with $C \leq 1,0<\alpha \leq 1$. Let $x, y \in A$ and choose a point $z \in A$ with $|x-z| \geq d(A) / 3$. If $|x-y| \leq|x-z|$, then

$$
\frac{|f x-f y|}{|f x-f z|} \leq C\left(\frac{|x-y|}{|x-z|}\right)^{\alpha} \leq C\left(\frac{3|x-y|}{d(A)}\right)^{\alpha}
$$

which yields the second inequality with $M=3^{\alpha} d(f A) d(A)^{-\alpha}$. Since $|f x-f y| \leq d(f A)$, this is also true if $|x-y| \geq|x-z| \geq d(A) / 3$.

6.16. Relative quasisymmetry. It is sometimes useful to consider the quasisymmetry of a map $f: X \rightarrow Y$ relative to a subset $A \subset X$. By a triple in $X$ we mean an ordered sequence $T=(x, a, b)$ of three distinct points in $X$. The ratio of a triple $T=(x, a, b)$ is the number

$$
\varrho(T)=\frac{|a-x|}{|b-x|} .
$$

If $f: X \rightarrow Y$ is an injective map, the image of a triple $T=(x, a, b)$ is the triple $f T=(f x, f a, f b)$ in $Y$.

Suppose that $A \subset X$. A triple $T=(x, a, b)$ in $X$ is said to be a triple in the pair $(X, A)$ if $x \in A$ or $\{a, b\} \subset A$. Equivalently, both distances in $\varrho(T)$ are distances from a point in $A$.

Let $\eta:[0, \infty) \rightarrow[0, \infty)$ be a homeomorphism. An embedding $f: X \rightarrow A$ is $\eta$ quasisymmetric relative to $A$, abbreviated rel $A$, if

$$
\varrho(f T) \leq \eta(\varrho(T))
$$

for each triple $T$ in $(X, A)$. Thus $\eta$-quasisymmetry rel $X$ is the same as ordinary $\eta$ quasisymmetry. In 6.17 we show that the case $\{a, b\} \subset A$ essentially follows from the case $x \in A$.

Relative quasisymmetry does not say much of the local behavior of the map far from A. For example, if $f: \bar{B}(1) \rightarrow \bar{B}(1)$ is any homeomorphism such that $f x=x$ for $1 / 2 \leq|x| \leq 1$, then $f$ is $\eta$-quasisymmetric rel $S(1)$ with a universal function $\eta$. 
6.17. Lemma. Suppose that $\eta:[0, \infty) \rightarrow[0, \infty)$ is a homeomorphism, that $A \subset X$, and that $f: X \rightarrow Y$ is an embedding such that $\varrho(f T) \leq \eta(\varrho(T))$ for each triple $T=(x, a, b)$ in $X$ with $x \in A$. Then $f$ is $\eta_{1}$-quasisymmetric rel $A$ with $\eta_{1}$ depending only on $\eta$.

Proof. Let $T=(x, a, b)$ be a triple in $X$ with $\{a, b\} \subset A$. For $T^{\prime}=(b, a, x)$ we have

$$
\varrho(T)=\frac{|a-x|}{|b-x|} \leq \frac{|a-b|+|b-x|}{|b-x|}=\varrho\left(T^{\prime}\right)+1,
$$

and similarly $\varrho\left(f T^{\prime}\right) \leq \varrho(f T)+1$. Hence $\varrho(f T) \leq \eta_{2}(\varrho(T))$ with $\eta_{2}(t)=\eta(t+1)+1$.

To complete the proof we assume that

$$
\varrho(T) \leq \frac{1}{2} \wedge\left(1+\eta^{-1}\left(\frac{1}{2}\right)^{-1}\right)^{-1},
$$

and show that $\varrho(f T) \leq 2 \eta(2 \varrho(T))$. For $T^{\prime \prime}=(a, x, b)$ we have

$$
\varrho\left(T^{\prime \prime}\right)=\frac{|a-x|}{|a-b|} \leq \frac{|a-x|}{|b-x|-|a-x|}=\frac{1}{1 / \varrho(T)-1} \leq 2 \varrho(T) \wedge \eta^{-1}\left(\frac{1}{2}\right) .
$$

Hence $\varrho\left(f T^{\prime \prime}\right) \leq 1 / 2$ and

$$
\varrho(f T) \leq \frac{|f a-f x|}{|f b-f a|-|f a-f x|} \leq 2 \varrho\left(f T^{\prime \prime}\right) \leq 2 \eta\left(\varrho\left(T^{\prime \prime}\right)\right) \leq 2 \eta(2 \varrho(T)) .
$$

6.18. Quasimöbius maps. By a quadruple in a metric space $X$ we mean an ordered sequence $Q=(a, b, c, d)$ of four distinct points in $X$. The cross ratio of $Q$ is the number

$$
\tau(Q)=\tau(a, b, c, d)=\frac{|a-b||c-d|}{|a-c||b-d|} .
$$

Permutating the points $a, b, c, d$ we can get at most 6 different numbers for $\tau(Q)$. The reader should be warned that in the literature at least 4 of them are called the cross ratio of $(a, b, c, d)$.

It is often convenient to consider cross ratios also in the extended space $\dot{X}=X \cup$ $\{\infty\}$. This is the Hausdorff space where $\infty \notin X$ and the neighborhoods of $\infty$ are the complements of closed bounded sets of $X$. If $Q=(a, b, c, d)$ is a quadruple in $\dot{X}$ and if one of the points $a, b, d, c$ is $\infty$, the cross ratio $\tau(Q)$ is defined by deleting the distances from $\infty$. For example,

$$
\tau(a, b, c, \infty)=\frac{|a-b|}{|a-c|}=\varrho(a, b, c) .
$$

The set quad $\dot{X}$ of all quadruples in $\dot{X}$ inherits a topology from $(\dot{X})^{4}$, and it is not difficult to show that $\tau$ : quad $\dot{X} \rightarrow \mathbf{R}$ is continuous.

Let $X$ and $Y$ be metric spaces, let $X_{0} \subset \dot{X}$, and let $\eta:[0, \infty) \rightarrow[0, \infty)$ be a homeomorphism. A map $f: X_{0} \rightarrow \dot{Y}$ is $\eta$-quasimöbius if $f$ is an embedding and

$$
\tau(f Q) \leq \eta(\tau(Q))
$$

for each quadruple $Q$ in $X_{0}$. If $f$ preserves all cross ratios, it is called a Möbius map.

It is sometimes convenient to define also quasisymmetry in the case where $f: X_{0} \rightarrow \dot{Y}$ is a map and $\infty \in X_{0}$. We say that such an $f$ is $\eta$-quasisymmetric if $f(\infty)=\infty$ and if $f \mid X_{0} \backslash\{\infty\}$ is an $\eta$-quasisymmetric map into $Y$. Such a map is always an embedding by $6.11(1)$. 
Observe that if $\infty \in X_{0}$ and if $f: X_{0} \rightarrow \dot{Y}$ is $\eta$-quasimöbius with $f(\infty)=\infty$, then $f$ is $\eta$-quasisymmetric by (6.19). Conversely, we show in 6.25 that an $\eta$-quasisymmetric map $f: X \rightarrow Y$ is always $\theta$-quasimöbius with $\theta=\theta_{\eta}$. Roughly speaking, the quasisymmetric maps are precisely the quasimöbius maps that fix the point at infinity.

The quasimöbius maps are more flexible than the quasisymmetric maps. For example, they may map bounded sets to unbounded sets. Moreover, several results admit a better formulation in terms of quasimöbius than quasisymmetric maps. For example, we shall later show that a $\varphi$-FQC map of a ball onto a ball is quasisymmetric, but this result is not quantitative unless we normalize the map in some way. On the other hand, the map is $\eta$-quasimöbius with $\eta=\eta_{\varphi}$.

We finally relativize the concept of quasimöbius. Let $A \subset X_{0} \subset \dot{X}$. A quadruple $Q=(a, b, c, d)$ of $X_{0}$ is said to be a quadruple in the pair $\left(X_{0}, A\right)$ if $\{a, d\} \subset A$ or $\{b, c\} \subset A$. Equivalently, all four distances in $\tau(Q)$ are (at least formally) distances from a point in $A$. An embedding $f: X_{0} \rightarrow \dot{Y}$ is $\eta$-quasimöbius relative to $A$, abbreviated rel $A$, if (6.20) holds for each quadruple $\mathrm{Q}$ in $\left(X_{0}, A\right)$. Thus $\eta$-quasimöbius rel $X_{0}$ is the same as ordinary $\eta$-quasimöbius.

6.21. Remarks. 1. Since $\tau(a, b, c, d)=\tau(b, a, d, c)$, an embedding $f: X_{0} \rightarrow \dot{Y}$ is $\eta$-quasimöbius rel $A$ as soon as (6.20) holds for every quadruple $Q=(a, b, c, d)$ in $X_{0}$ such that $\{a, d\} \subset A$.

2. Since the cross ratio is more complicated than the ratio, the quasisymmetric maps are technically easier to deal with than the quasimöbius maps. However, it is often possible to convert a cross ratio to a ratio by mapping one of the points to $\infty$ by an auxiliary inversion and applying Theorem 6.22 below. We extend the inversion $u(x)=x /|x|^{2}$ to a self homeomorphism $u: \dot{E} \rightarrow \dot{E}$ by setting $u(0)=\infty$ and $u(\infty)=0$.

6.22. THEOREM. If $E$ is a Hilbert space, then the inversion $u: \dot{E} \rightarrow \dot{E}$ is Möbius. In the general case $u$ is $\eta$-quasimöbius with $\eta(t)=81$.

Proof. This follows at once from (5.10) and (5.9). The bound 81 is best possible.

We next give some basic properties of quasimöbius maps. The following result is obvious:

6.23. THEOREM. (1) If $f$ is $\eta_{1}$-quasimöbius and if $g$ is $\eta_{2}$-quasimöbius and if $g f$ is defined, it is $\eta$-quasimöbius with $\eta(t)=\eta_{2}\left(\eta_{1}(t)\right)$.

(2) If $f: X_{0} \rightarrow \dot{Y}$ is $\eta$-quasimöbius, then $f^{-1}: f X_{0} \rightarrow X_{0}$ is $\eta^{\prime}$-quasimöbius with $\eta^{\prime}(t)=\eta^{-1}\left(t^{-1}\right)^{-1}$.

6.24. TheOREM. Suppose that $X_{0} \subset \dot{E}$ and that $f: X_{0} \rightarrow \dot{E}^{\prime}$ is $\eta$-quasimöbius. Then $f$ extends to an $\eta$-quasimöbius map $g: \bar{X}_{0} \rightarrow \dot{E}^{\prime}$.

Proof. It suffices to find an extension $g$ which is an embedding, since the $\eta$-quasimöbius property follows then by continuity. If $\infty \in X_{0}$ and if $f(\infty)=\infty$, then $f$ is quasisymmetric, and the extension $g$ is given by 6.12 . The general case reduces to this by means of auxiliary inversions. 
6.25. TheOREM. If $f: X \rightarrow Y$ is $\eta$-quasisymmetric rel $A \subset X$, then $f$ is $\theta$ quasimöbius rel $A$ with $\theta=\theta_{\eta}$.

Proof. Let $Q=(a, b, c, d)$ be a quadruple in $X$ with $\{a, d\} \subset A$. It suffices to find an estimate $\tau(f Q) \leq \theta(\tau(Q))$ where $\theta(t) \rightarrow 0$ as $t \rightarrow 0$.

Setting $\tau=\tau(Q)$ and

$$
\varepsilon=\frac{|a-b|}{|a-c|}, \delta=\frac{|c-d|}{|a-c|}
$$

we have

$$
\frac{\tau}{\varepsilon}=\frac{|c-d|}{|b-d|}, \frac{\tau}{\delta}=\frac{|a-b|}{|b-d|}
$$

Since $\tau=\tau(d, c, b, a)$, we may assume, by symmetry, that $\varepsilon \leq \tau / \varepsilon$, and hence $\varepsilon \leq \sqrt{\tau}$. Since $f$ is $\eta$-quasisymmetric rel $A$, we have

$$
\begin{aligned}
& \tau(f Q) \leq \eta(\varepsilon) \eta(\tau / \varepsilon), \\
& \tau(f Q) \leq \eta(\delta) \eta(\tau / \delta) .
\end{aligned}
$$

Furthermore,

$$
|d-c| \leq|d-b|+|b-a|+|a-c|=\varepsilon|c-d| / \tau+\varepsilon|a-c|+|a-c| .
$$

If $\varepsilon<\tau$, this implies

$$
\delta \leq \tau \frac{1+\varepsilon}{\tau-\varepsilon}
$$

Moreover, since

$$
|a-c| \leq|a-b|+|b-d|+|d-c|=\varepsilon|a-c|+\varepsilon|c-d| / \tau+|d-c|,
$$

we get

$$
\delta \geq \tau \frac{1-\varepsilon}{\tau+\varepsilon}
$$

We consider three cases.

Case 1. $\varepsilon \geq 1 / 2$. Then $\tau / \varepsilon \leq 2 \tau$, and (1) gives $\tau(f Q) \leq \eta(\sqrt{\tau}) \eta(2 \tau)$.

Case 2. $\varepsilon \geq \tau / 2$. Now $\tau / \varepsilon \leq 2$, and we get $\tau(f Q) \leq \eta(\sqrt{\tau}) \eta(2)$.

Case 3 . $\varepsilon \leq 1 / 2$ and $\varepsilon \leq \tau / 2$. By (3) and (4) we have

$$
\delta \leq \tau \frac{3 / 2}{\tau / 2}=3, \frac{\tau}{\delta} \leq \frac{3 \tau / 2}{1 / 2}=3 \tau .
$$

Hence (2) yields $\tau(f Q) \leq \eta(3) \eta(3 \tau)$.

6.26. Bounded spaces. A quasimöbius map need not be quasisymmetric. For example, a Möbius map of a ball in $R^{n}$ onto a half space is not quasisymmetric. A Möbius map between bounded sets is quasisymmetric, but this result is not quantitative. For example, the unit ball of $R^{n}$ can be mapped onto itself by a Möbius map so that $|f(0)|$ is arbitrarily close to 1 . In order to get a quantitative result, one must normalize the map in some way. For general metric spaces we need a normalization in three points. For homeomorphisms $f: G \rightarrow G^{\prime}$, it is sufficient to assume that for some $x_{0} \in G, f x_{0}$ is not too close to $\partial G^{\prime}$. We prove these results in the relative setting; the absolute cases are obtained as obvious corollaries. 
We first give characterizations for relative quasisymmetry and quasimöbius.

6.27. TheOrem. Suppose that $f: X \rightarrow Y$ is an embedding, that $A \subset X$, and that $\eta:[0, \infty) \rightarrow[0, \infty)$ is an increasing function (not necessarily with $\eta(0)=0$ ) such that

(1) $\varrho(f T) \leq \eta(\varrho(T))$ for each triple $T=(x, a, b)$ in $X$ with $x \in A$,

(2) $\varrho\left(f^{-1} T^{\prime}\right) \leq \eta\left(\varrho\left(T^{\prime}\right)\right)$ for each triple $T^{\prime}=\left(x^{\prime}, a^{\prime}, b^{\prime}\right)$ in $f X$ with $x^{\prime} \in f A$.

Then $f$ is $\eta_{1}$-quasisymmetric rel $A$ with $\eta_{1}$ depending only on $\eta$.

Proof. Let $T=(x, a, b)$ be a triple in $X$ with $x \in A$. By 6.17 , it suffices to find a homeomorphism $\eta_{1}:[0, \infty) \rightarrow[0, \infty)$ such that $\varrho(f T) \leq \eta_{1}(\varrho(T))$. Replacing $\eta$ by a larger function we may assume that $\eta$ is a homeomorphism onto $\left[r_{0}, \infty\right), r_{0}>0$. Setting $t_{0}=1 / r_{0}$ we define an increasing homeomorphism $\eta_{0}:\left(0, t_{0}\right) \rightarrow(0, \infty)$ by $\eta_{0}(t)=\eta^{-1}\left(t^{-1}\right)^{-1}$. Suppose that $\varrho(T)<t_{0}$. Applying (2) to the triple $T^{\prime}=(f x, f b, f a)$ gives $1 / \varrho(T) \leq \eta\left(\rho\left(T^{\prime}\right)\right)$, which implies $\left.\varrho(f T)\right) \leq \eta_{0}(\varrho(T))$. Hence the assertion holds with $\eta_{1}$ defined by $\eta_{1}(t)=\eta(t) \wedge \eta_{0}(t)$ for $t<t_{0}$ and by $\eta_{1}(t)=\eta(t)$ for $t \geq t_{0}$.

6.28. Theorem. Suppose that $A \subset X_{0} \subset \dot{X}$, that $f: X_{0} \rightarrow \dot{Y}$ is an embedding and that $\eta:[0, \infty) \rightarrow[0, \infty)$ is an increasing function such that

(1) $\tau(f Q) \leq \eta(\tau(Q))$ for each quadruple $Q=(a, b, c, d)$ with $\{a, d\} \subset A$,

(2) $\tau\left(f^{-1} Q^{\prime}\right) \leq \eta\left(\tau\left(Q^{\prime}\right)\right)$ for each quadruple $Q^{\prime}=\left(a^{\prime}, b^{\prime}, c^{\prime}, d^{\prime}\right)$ with $\left\{a^{\prime}, d^{\prime}\right\} \subset f A$.

Then $f$ is $\eta_{1}$-quasimöbius rel $A$ with $\eta_{1}$ depending only on $\eta$.

PROOF. In view of 6.21.1, the proof is an obvious modification of the proof of 6.27 .

6.29. Theorem. Suppose that $X$ and $Y$ are bounded spaces, that $A \subset X$, and that $f: X \rightarrow Y$ is $\theta$-quasimöbius rel $A$. Suppose also that $\lambda>0, z_{1} \in X$, and $z_{2}, z_{3} \in A$ are such that

$$
\left|z_{i}-z_{j}\right| \geq d(X) / \lambda,\left|f z_{i}-f z_{j}\right| \geq d(Y) / \lambda
$$

for $i \neq j$. Then:

(1) There is a homeomorphism $\mu=\mu_{\theta, \lambda}:[0, \infty) \rightarrow[0, \infty)$ such that

$$
\frac{|f x-f y|}{d(Y)} \leq \mu\left(\frac{|x-y|}{d(X)}\right) \quad \text { for all } x \in A, y \in X \text {. }
$$

(2) $f$ is $\eta$-quasisymmetric rel $A$ with $\eta$ depending only on $\theta$ and $\lambda$.

Proof. We may assume that $f$ is a homeomorphism and that $f^{-1}: Y \rightarrow X$ is $\theta$-quasimöbius rel $f A$. We normalize the situation so that $d(X)=d(Y)=\lambda$ replacing the metric $|a-b|$ of $X$ by $\lambda|a-b| / d(X)$ and similarly in $Y$.

(1) Let $x \in A, y \in X$, and set $r=|x-y|, r^{\prime}=|f x-f y|$. It suffices to find an estimate $r^{\prime} \leq \mu(r)$ where $\mu(r) \rightarrow 0$ as $r \rightarrow 0$. We consider three cases:

Case 1. $\left|x-z_{1}\right| \leq 1 / 2, r \leq 1 / 4$. Now $\left|x-z_{j}\right| \geq 1 / 2$ and $\left|y-z_{j}\right| \geq 1 / 4$ for $j=2,3$. For $Q=\left(x, y, z_{2}, z_{3}\right)$ we thus have $\tau(Q) \leq 8 \lambda r$ and $\tau(f Q) \geq r^{\prime} / \lambda^{2}$. Since $\left\{x, z_{3}\right\} \subset A$, this yields $r^{\prime} \leq \lambda^{2} \theta(8 \lambda r)$.

Case 2. $r \geq 1 / 4$. Now $r^{\prime} \leq \lambda \leq 4 \lambda r$.

Case 3. $\left|x-z_{1}\right| \geq 1 / 2$. There is $z \in\left\{z_{2}, z_{3}\right\}$ with $|y-z| \geq 1 / 2$. For $Q=\left(x, y, z_{1}, z\right)$ we obtain $\tau(Q) \leq 4 \lambda r$ and $\tau(f Q) \geq r^{\prime} / \lambda^{2}$. Since $\{x, z\} \subset A$, we obtain $r^{\prime} \leq \lambda^{2} \theta(4 \lambda r)$. 
(2) Define $\psi:[0, \infty) \rightarrow[0, \infty)$ by $\psi(t)=\lambda \mu(t / \lambda)$. Then

$$
\psi^{-1}(|x-y|) \leq|f x-f y| \leq \psi(|x-y|) \quad \text { for all } x \in A, y \in X .
$$

Let $T=(x, a, b)$ be a triple in $(X, A)$. By symmetry and by 6.27 , it suffices to find an estimate

$$
\varrho(f T) \leq \eta(\varrho(T))
$$

for some increasing $\eta:[0, \infty) \rightarrow[0, \infty)$ depending only on $\theta$ and $\lambda$. Since $\left|z_{2}-z_{3}\right| \geq 1$, we may assume that $\left|a-z_{2}\right| \geq 1 / 2$. We consider three cases.

Case 1. $|a-x| \geq 1 / 4$. Now $|b-x| \geq 1 / 4 \varrho(T)$. Since $b \in A$ or $x \in A$, we have $|f b-f x| \geq \psi^{-1}(1 / 4 \varrho(T))$, and hence (6.30) holds with $\eta(t)=\lambda / \psi^{-1}(1 / 4 t)$.

Case 2. $\left|b-z_{2}\right| \geq 1 / 8$. In this case we have $\left|f b-f z_{2}\right| \geq \psi^{-1}(1 / 8)$. The quadruple $Q=\left(x, a, b, z_{2}\right)$ is in $(X, A)$ and $\tau(Q) \leq 2 \lambda \varrho(T)$. Since $\tau(f Q) \geq \psi^{-1}(1 / 8) \varrho(f T) / \lambda$, we obtain (6.30) with

$$
\eta(t)=\frac{\lambda \theta(2 \lambda t)}{\psi^{-1}(1 / 8)}
$$

Case 3. $|a-x| \leq 1 / 4$ and $\left|b-z_{2}\right| \leq 1 / 8$. Now

$$
|b-x| \geq\left|a-z_{2}\right|-|a-x|-\left|z_{2}-b\right| \geq 1 / 2-1 / 4-1 / 8=1 / 8 .
$$

Hence $|f b-f x| \geq \psi^{-1}(1 / 8)$, which implies (6.30) with the constant function $\eta(t)=$ $\lambda / \psi^{-1}(1 / 8)$.

6.31. Theorem. Suppose that $G$ and $G^{\prime}$ are bounded domains and that $c \geq 1$. Suppose also that $x_{0} \in G$ and $x_{0}^{\prime} \in G^{\prime}$ are points with

$$
d(G) \leq c \delta\left(x_{0}\right), \quad d\left(G^{\prime}\right) \leq c \delta^{\prime}\left(x_{0}^{\prime}\right) .
$$

Let $f: \bar{G} \rightarrow \overline{G^{\prime}}$ be a homeomorphism such that $f x_{0}=x_{0}^{\prime}$ and $f \partial G=\partial G^{\prime}$, and let $\partial G \subset A \subset \bar{G}$. If $f$ is $\theta$-quasimöbius rel $A$, then $f$ is $\eta$-quasisymmetric rel $A$ with $\eta=\eta_{\theta, c}$.

Proof. Write $z_{1}=x_{0}, M=d(G)=d(\partial G)$ and $M^{\prime}=d\left(G^{\prime}\right)=d\left(\partial G^{\prime}\right)$. Choose points $z_{2}, z_{3} \in \partial G$ with $\left|z_{2}-z_{3}\right| \geq M / 2$. It suffices to show that the points $z_{1}, z_{2}, z_{3}$ satisfy the conditions of 6.29 with some $\lambda=\lambda(\theta, c)$.

Since

$$
\left|z_{1}-z_{2}\right| \geq \delta\left(x_{0}\right) \geq M / c,\left|z_{1}-z_{3}\right| \geq M / c,\left|z_{2}-z_{3}\right| \geq M / 2,
$$

the first condition of 6.29 is true with $\lambda=c \vee 2$. For $j=2,3$ we have

$$
\left|f z_{j}-f z_{1}\right| \geq \delta^{\prime}\left(x_{0}\right) \geq M^{\prime} / c .
$$

It remains to find a lower bound for $\left|f z_{2}-f z_{3}\right| / M^{\prime}$.

Choose $z_{4} \in \partial G$ with $\left|f z_{4}-f z_{3}\right| \geq M^{\prime} / 3$. The quadruple $Q=\left(z_{2}, z_{1}, z_{3}, z_{4}\right)$ is in $(\bar{G}, \partial G)$ and hence in $(\bar{G}, A)$. We have

$$
\tau(Q) \leq 2 c, \tau(f Q) \geq \frac{M^{\prime}}{3 c\left|f z_{2}-f z_{3}\right|} .
$$

Since $f$ is $\theta$-quasimöbius rel $A$, we obtain the desired estimate $M^{\prime} \leq 3 c \theta(2 c)\left|f z_{2}-f z_{3}\right|$. 
6.33. Theorem. Suppose that $G$ and $G^{\prime}$ are bounded domains, and that $x_{0} \in G, x_{0}^{\prime} \in$ $G^{\prime}$ and $c \geq 1$ satisfy (6.32). Let $f: G \rightarrow G^{\prime}$ be a $\theta$-quasimöbius homeomorphism with $f x_{0}=x_{0}^{\prime}$. Then $f$ is $\eta$-quasisymmetric with $\eta=\eta_{\theta, c}$.

Proof. By $6.24, f$ extends to a $\theta$-quasimöbius map $\bar{f}: \bar{G} \rightarrow \overline{G^{\prime}}$. The theorem follows from 6.31 by choosing $A=\bar{G}$.

6.34. Example. Let $f: B\left(x_{0}, r\right) \rightarrow B\left(x_{0}^{\prime}, r^{\prime}\right)$ be a $\theta$-quasimöbius homeomorphism with $f x_{0}=x_{0}^{\prime}$. Then $f$ is $\eta$-quasisymmetric with $\eta=\eta_{\theta}$.

6.35. Notes. Quasisymmetric maps $f: \mathbf{R} \rightarrow \mathbf{R}$ were introduced in 1956 by A. Beurling and L. Ahlfors $[\mathrm{BA}]$, who proved that they are precisely the homeomorphisms that extend to a quasiconformal map of the upper half plane. The notion was generalized to metric spaces in $\left[\mathrm{TV}_{1}\right]$ in 1980. Quasimöbius maps between Jordan curves in $R^{2}$ were considered by S. Rickman [Ri] in 1969 and in arbitrary metric spaces by the author [Vä in 1985. Relative quasisymmetry and quasimöbius maps were introduced in [II].

\section{FQC, solid and coarsely QH maps}

7.1. Summary. We analyze the concepts given in the title of the section. We start by characterizing solidity in terms of another property called relativity. Next we show that the free $\varphi$-quasiconformality is quantitatively equivalent to seven other properties. Some of these, however, are only slight variations of each other. We also consider the relations between free quasiconformality and the quasisymmetric and quasimöbius properties.

7.2. Definitions. Let $0<t_{0} \leq 1$ and let $\theta:\left[0, t_{0}\right) \rightarrow[0, \infty)$ be a homeomorphism. Let $f: G \rightarrow G^{\prime}$ be a homeomorphism with $G \subsetneq E, G^{\prime} \subsetneq E^{\prime}$. Recall the notation $\delta(x)=d(x, \partial G), \delta^{\prime}(y)=d\left(y, \partial G^{\prime}\right)$. We say that $f$ is $\left(\theta, t_{0}\right)$-relative if

$$
\frac{|f x-f y|}{\delta^{\prime}(f x)} \leq \theta\left(\frac{|x-y|}{\delta(x)}\right)
$$

whenever $x, y \in G$ and $|x-y|<t_{0} \delta(x)$. If $t_{0}=1$, we say that $f$ is $\theta$-relative.

We shall show in 7.6 that these properties are quantitatively equivalent to the second inequality

$$
k^{\prime}(f x, f y) \leq \varphi(k(x, y))
$$

of the definition (4.4) of $\varphi$-solidity. We say that a homeomorphism $f: G \rightarrow G^{\prime}$ is $\varphi$ semisolid if (7.3) holds for all $x, y \in G$. Thus $f$ is $\varphi$-solid if and only if $f$ and $f^{-1}$ are $\varphi$-semisolid.

7.4. Example. We show that semisolidity does not imply solidity. Let $G$ be the upper half plane of $R^{2}$ and define a homeomorphism $f: G \rightarrow G$ as follows:

$$
\begin{cases}f x=x & \text { for } x_{1} \leq 0, \\ f x=\left(x_{1}, x_{1} x_{2}+x_{2}\right) & \text { for } x_{1} \geq 0,0<x_{2} \leq 1, \\ f x=\left(x_{1}, x_{1}+x_{2}\right) & \text { for } x_{1} \geq 0, x_{2} \geq 1 .\end{cases}
$$

Consider a point $x$ in the half strip $x_{1}>0,0<x_{2}<1$. The estimate $(5.20)$ gives

$$
\left|f^{\prime}(x)\right|^{2} \leq 1+x_{2}^{2}+\left(1+x_{1}\right)^{2} \leq 2+\left(1+x_{1}\right)^{2} .
$$


Since $\delta(x)=x_{2}$ and $\delta^{\prime}(f x)=x_{1} x_{2}+x_{2}$, this and 5.4 imply

$$
L_{k}(x, f)^{2} \leq \frac{2+\left(1+x_{1}\right)^{2}}{\left(1+x_{1}\right)^{2}} \leq 3 .
$$

This estimate is also valid for $x_{1}>0, x_{2} \geq 1$, and hence for all $x \in G$. By 5.5 and 3.4(2) it follows that $f$ is $\sqrt{3}$-Lipschitz in the QH metric and hence $\varphi$-semisolid with $\varphi(t)=t \sqrt{3}$.

We show that $f^{-1}$ is not semisolid. Let $a>2$ and consider the points $x=(a, 1)$ and $y=(a+1,1)$. Then $3.7(1)$ and 3.9 give

$$
k(x, y) \geq \log 2, k(f x, f y) \leq \frac{2 \sqrt{2}}{a+1} .
$$

Since $a$ can be arbitrarily large, $f^{-1}$ is not uniformly continuous in the QH metric.

However, the following question is open:

7.5. Problem. Is full $\varphi$-semisolidity quantitatively equivalent to full $\varphi$-solidity, that is, to free $\varphi$-quasiconformality?

In $R^{n}$ these properties are $n$-quantitatively equivalent; see 7.13 . We show in 7.14 that the answer is affirmative for maps of the whole space.

7.6. Theorem. Let $G \neq E, G^{\prime} \neq E^{\prime}$, and let $f: G \rightarrow G^{\prime}$ be a homeomorphism. Then the following properties are quantitatively equivalent:

(1) $f$ is $\theta$-relative,

(2) $f$ is $\left(\theta, t_{0}\right)$-relative,

(3) $f$ is $\varphi$-semisolid.

Proof. Since (1) implies (2) trivially, it suffices to prove that $(2) \Rightarrow(3) \Rightarrow(1)$.

$(2) \Rightarrow(3)$ : Choose $t_{1}<t_{0}$ such that $0<t_{1} \leq 1 / 2$ and $\theta\left(t_{1}\right) \leq 1 / 2$. Let $x, y \in G$ be points with $k(x, y) \leq t_{1} / 2$. Then 3.9 gives $|x-y| \leq t_{1} \delta(x)$. By (2) we obtain

$$
|f x-f y| \leq \theta\left(t_{1}\right) \delta^{\prime}(f x) \leq \delta^{\prime}(f x) / 2 .
$$

Again by 3.9 and (2) this gives

$$
k^{\prime}(f x, f y) \leq \frac{2|f x-f y|}{\delta^{\prime}(f x)} \leq 2 \theta\left(\frac{|x-y|}{\delta(x)}\right) \leq 2 \theta(2 k(x, y)) .
$$

Hence $f$ is $\left(\varphi, t_{1} / 2\right)$-uniformly continuous in the QH metric with $\varphi(t)=2 \theta(2 t)$. Since $(G, k)$ is 2-quasiconvex, (3) follows from 2.5.

$(3) \Rightarrow(1)$ : Suppose that $x, y \in G,|x-y|=t \delta(x), 0<t<1$. Define homeomorphisms $\theta_{0}:[0,1) \rightarrow[0, \infty)$ and $\psi:[0, \infty) \rightarrow[0, \infty)$ by $\theta_{0}(t)=t /(1-t)$ and $\psi(t)=e^{t}-1$. Applying parts (3) and (2) of 3.7 we get

$$
k(x, y) \leq \theta_{0}(t), \frac{|f x-f y|}{\delta^{\prime}(f x)} \leq \psi\left(k^{\prime}(f x, f y)\right) .
$$

Hence (1) holds with $\theta=\psi \varphi \theta_{0}$.

7.7. Corollary. For a homeomorphism $f: G \rightarrow G^{\prime}$, the following conditions are quantitatively equivalent:

(1) $f$ and $f^{-1}$ are $\theta$-relative,

(2) $f$ and $f^{-1}$ are $\left(\theta, t_{0}\right)$-relative,

(3) $f$ is $\varphi$-solid. 
7.8. Definitions. We show in 7.9 that full $\varphi$-semisolidity is quantitatively equivalent to seven other conditions. As a corollary we obtain seven alternative characterizations for free quasiconformality. We first introduce some terminology.

Let $0<q<1$ and let $f: G \rightarrow G^{\prime}$ be a homeomorphism. We say that $f$ is $q$-locally $\eta$-quasisymmetric if $f \mid B(x, q r)$ is $\eta$-quasisymmetric whenever $B(x, r) \subset G$. If $G \neq E$, this means that $f \mid B(x, q \delta(x))$ is $\eta$-quasisymmetric for each $x \in G$. If $G=E$, this means that $f$ is $\eta$-quasisymmetric. The weak q-local $H$-quasisymmetry is defined analogously.

For any ball $B=B(x, r)$ and for $\alpha>0$ we write $\alpha B=B(x, \alpha r)$. Let $1<\alpha \leq \beta$ and let $M>0$. We say that a homeomorphism $f: G \rightarrow G^{\prime}$ has the $(M, \alpha, \beta)$-ring property if

$$
d(f \bar{B}) \leq M d(f \bar{B}, \partial f \alpha B)
$$

whenever $B$ is a ball such that $\beta B \subset G$.

7.9. TheOREM. For a homeomorphism $f: G \rightarrow G^{\prime}$, the following conditions are quantitatively equivalent:

(1) $f$ is fully $\varphi$-semisolid.

(2) $f$ is fully $C$-coarsely $M$-Lipschitz in the $\mathrm{QH}$ metric.

(3) For each $0<q<1$ there is $\eta$ such that $f$ is q-locally $\eta$-quasisymmetric.

(4) $f$ is q-locally $\eta$-quasisymmetric.

(5) For each $0<q<1$ there is $H$ such that $f$ is q-locally weakly $H$-quasisymmetric.

(6) $f$ is q-locally weakly $H$-quasisymmetric.

(7) For each $\alpha>1$ there is $M$ such that $f$ has the $(M, \alpha, \alpha)$-ring property.

(8) $f$ has the $(M, \alpha, \beta)$-ring property.

Proof. We first remark that the data in the various conditions are as follows: (1) $\varphi$, (2) $(M, C),(3) q \mapsto \eta,(4)(q, \eta),(5) q \mapsto H,(6)(q, H),(7) \alpha \mapsto M,(8)(M, \alpha, \beta)$.

We shall verify the implications indicated by the following diagram.

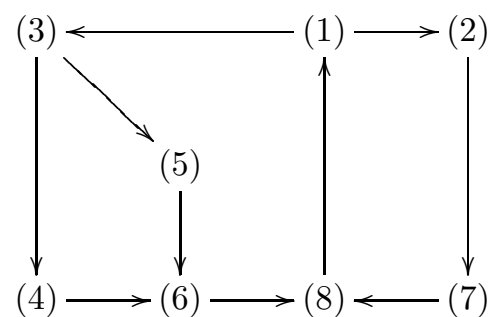

The quantitative implications $(3) \Rightarrow(4) \Rightarrow(6),(3) \Rightarrow(5) \Rightarrow(6)$ and $(7) \Rightarrow(8)$ are trivial, and $(1) \Rightarrow(2)$ follows from 2.3 and $3.4(2)$. It remains to prove 4 implications.

$(1) \Rightarrow(3)$ : We first show that $f$ is $\frac{1}{3}$-locally $\eta$-quasisymmetric with some $\eta=\eta_{\varphi}$. Assume that $B$ is a ball such that $3 B \subset G$ and that $x, a, b \in B$ with $|a-x|=t|b-x|, t>0$. We must find an estimate

$$
|f a-f x| \leq \eta(t)|f b-f x|,
$$

where $\eta(t) \rightarrow 0$ as $t \rightarrow 0$. We consider three cases. 
Case 1. $t \leq 2 / 3$. Setting $D=G \backslash\{b\}$ we have $f D=G^{\prime} \backslash\{f b\}$. Since $f_{D}$ is $\varphi$-semisolid, $f_{D}$ is $\theta$-relative with $\theta=\theta_{\varphi}$ by 7.6. Since $|x-a|=t|b-x|=t \delta_{D}(x)$, we have

$$
|f a-f x| \leq \theta(t) \delta_{f D}(f x) \leq \theta(t)|f b-f x|,
$$

and hence (7.10) holds with $\eta(t)=\theta(t)$.

Case 2. $2 / 3 \leq t \leq 1$. The point $z=2 a / 3+x / 3$ lies in $B$. Since $|a-z|=|x-z| / 2$, Case 1 gives

$$
|f a-f z| \leq \theta(1 / 2)|f x-f z| .
$$

Since $|z-x|=2|a-x| / 3 \leq 2|b-x| / 3$, Case 1 yields

$$
|f z-f x| \leq \theta(2 / 3)|f b-f x| \text {. }
$$

Hence

$$
|f a-f x| \leq|f a-f z|+|f z-f x| \leq(1+\theta(1 / 2)) \theta(2 / 3)|f b-f x| .
$$

Case 3. $t>1$. It follows from Cases 1 and 2 that $f \mid B$ is weakly $H$-quasisymmetric with $H=H(\varphi)$. Since $B$ is convex, 6.5 gives an estimate $|f a-f x| \leq M(t, H)|f b-f x|$.

We have proved that $f$ is $\frac{1}{3}$-locally $\eta$-quasisymmetric with $\eta=\eta_{\varphi}$. Of course, $f$ is $q$-locally $\eta$-quasisymmetric for $0<q<1 / 3$ with the same $\eta$.

Next let $1 / 3 \leq q<1$. Let $B$ be a ball with $3 B \subset G$. We show that $f \mid 3 q B$ is $\eta$ quasisymmetric. Let $g:[0,3] \rightarrow[0,3]$ be the increasing homeomorphism that is affine on $[0,1]$ and on $[1,3]$ with $g(1)=3 q$. Then $q$ is $M$-bilipschitz with $M=M(q)$. Applying 5.22 and auxiliary similarities we find a $3 M$-bilipschitz homeomorphism $h_{0}: 3 B \rightarrow 3 B$ with $h_{0} B=3 q B$. We extend $h_{0}$ by identity to a $3 M$-bilipschitz homeomorphism $h: G \rightarrow G$. By $5.16, h$ is fully $9 M^{2}$-QH. Hence the map $f_{1}: f h: G \rightarrow G^{\prime}$ is fully $\psi$-semisolid with $\psi(t)=\varphi\left(9 M^{2} t\right)$. By the first part of the proof, $f_{1} \mid B$ is $\eta$-quasisymmetric with $\eta=\eta_{\varphi, q}$. Since $h \mid B$ is a similarity onto $3 q B, f \mid 3 q B$ is $\eta$-quasisymmetric.

$(6) \Rightarrow(8)$ : We show that $(8)$ is true with $\alpha=2, \beta=3 / q>3$ and $M=2 H^{2}$. Let $x \in G$ and $r>0$ be such that $B(x, \beta r) \subset G$. Since $f \mid B(x, 3 r)$ is weakly quasisymmetric, it follows from 6.13 that $f \bar{B}$ is closed in $E^{\prime}$ for every ball $B \subset B(x, 2 r)$. Hence $f \partial B=\partial f B$, which implies that

$$
d(f \bar{B})=d(f \partial B), d(f \bar{B}, \partial f B(x, 2 r))=d(f \partial B, f S(x, 2 r)) .
$$

Let $a, b, y \in S(x, r)$ and $z \in S(x, 2 r)$. It suffices to show that

$$
|f a-f b| \leq M|f y-f z| \text {. }
$$

Since $f$ is weakly $H$-quasisymmetric in $B(x, 3 r)$ and since $|a-x|=|b-x|=|y-x|$, we have

$$
|f a-f b| \leq|f a-f x|+|f x-f b| \leq 2 H|f y-f x| .
$$

Moreover, since $|y-z| \geq|z-x|-|y-x|=2 r-r=r=|y-x|$, we get $|f y-f x| \leq H|f y-f z|$, and (7.11) follows.

$(8) \Rightarrow(1)$ : For each domain $D \subsetneq G$, the map $f_{D}: D \rightarrow f D$ satisfies (8). Hence it suffices to show that $f$ is $\varphi$-semisolid with $\varphi=\varphi_{M, \alpha, \beta}$, assuming that $G \neq E, G^{\prime} \neq E^{\prime}$. By 7.6 it suffices to show that if $x, y \in G$ with $|x-y| \leq \delta(x) / \alpha^{3} \beta$, then

$$
\frac{|f x-f y|}{\delta^{\prime}(f x)} \leq \theta\left(\frac{|x-y|}{\delta(x)}\right)
$$


with some function $\theta:\left[0,1 / \alpha^{3} \beta\right) \rightarrow[0, \infty)$ depending only on $(M, \alpha, \beta)$ such that $\theta(t) \rightarrow$ 0 as $t \rightarrow 0$.

Suppose that $|x-y|=t \delta(x), 0<t<1 / \alpha^{3} \beta$. Let $m$ be the largest integer with $\alpha^{m} \beta t<1$; then $m \geq 3$. Set $r_{j}=\alpha^{j} t \delta(x)$ and $D_{j}=B\left(x, r_{j}\right)$ for $0 \leq j \leq m$. Choose a point $z \in \partial G^{\prime}$ with $|z-f x| \leq 2 \delta^{\prime}(f x)$. Since $B\left(x, \beta r_{m}\right) \subset G$, it follows from (8) that $d\left(f \bar{D}_{j}, \partial G^{\prime}\right)>0$ for $j \leq m$. Hence we can choose for each $j=1, \ldots, m$ the last point $y_{j}$ of the line segment $[f x, z)$ in $\partial f D_{j}$. Set $\lambda=\left|y_{1}-f x\right|$. Then (8) gives

$$
\lambda \leq\left|y_{j-1}-f x\right| \leq d\left(f \bar{D}_{j-1}\right) \leq M d\left(f \bar{D}_{j-1}, \partial f D_{j}\right) \leq M\left|y_{j}-y_{j-1}\right|
$$

for $2 \leq j \leq m$. Summing over these $j$ we obtain

$$
(m-1) \lambda \leq M\left|y_{n}-y_{1}\right| \leq M|z-f x| \leq 2 M \delta^{\prime}(f x) .
$$

On the other hand, (8) yields

$$
|f x-f y| \leq d\left(f \bar{D}_{0}\right) \leq M d\left(f \bar{D}_{0}, \partial f D_{1}\right) \leq M \lambda .
$$

Hence

$$
\frac{|f x-f y|}{\delta^{\prime}(f x)} \leq \frac{2 M^{2}}{m-1}
$$

Since $\alpha^{m+1} \beta t \geq 1$, we have

$$
m+1 \geq \frac{\log (1 / \beta t)}{\log \alpha} .
$$

Here $\alpha^{3} \beta t<1$ implies $3 \log \alpha<\log (1 / \beta t)$, and hence

$$
m-1 \geq \frac{\log (1 / \beta t)-2 \log \alpha}{\log \alpha} \geq \frac{\log (1 / \beta t)}{3 \log \alpha} .
$$

Combining these inequalities yields the desired estimate

$$
\frac{|f x-f y|}{\delta^{\prime}(f x)} \leq \frac{6 M^{2} \log \alpha}{\log (1 / \beta t)}=\theta(t) .
$$

$(2) \Rightarrow(7)$ : Assume that (2) holds with some $M>0$ and $C \geq 0$, and let $\alpha>1$. We must find $M_{0}=M_{0}(M, C, \alpha)$ such that $f$ has the $\left(M_{0}, \alpha, \alpha\right)$-ring property.

Let $B$ be a ball such that $D=\alpha B \subset G$. Let $a \in \bar{B}$ and choose $b \in \bar{B}$ with $|f a-f b| \geq$ $d(f \bar{B}) / 3$. Then $k_{D}(a, b) \leq 2 /(\alpha-1)$ by $3.7(3)$. By $(2)$ and 3.7(1) we obtain

$$
\log \frac{|f a-f b|}{\delta_{f D}(f a)} \leq k_{f D}(f a, f b) \leq M k_{D}(a, b)+C \leq \frac{2 M}{\alpha-1}+C=M_{1} .
$$

Hence

$$
d(f \bar{B}) \leq 3 e^{M_{1}} \delta_{f D}(f a)
$$

for each $a \in \bar{B}$, and we can choose $M_{0}=3 e^{M_{1}}$.

7.12. Corollary. For a homeomorphism $f: G \rightarrow G^{\prime}$ the following eight conditions are quantitatively equivalent:

(1) $f$ is $\varphi$-FQC.

(2) $f$ is fully $C$-coarsely $M$-QH.

$(j), 3 \leq j \leq 8$. $f$ and $f^{-1}$ satisfy the condition $(j)$ of 7.9 . 
7.13. Theorem. Suppose that $G$ and $G^{\prime}$ are domains in $R^{n}$ and that $f: G \rightarrow G^{\prime}$ is fully $\varphi$-semisolid. Then $f$ is $K$-quasiconformal with $K=K(\varphi)$.

Proof. By $7.9(5)$ there is $H=H(\varphi)$ such that $f$ is $\frac{1}{2}$-locally weakly $H$-quasisymmetric. Hence $f$ is $H$-quasiconformal by our definition in 1.1 .

7.14. TheOrem. Suppose that $f: E \rightarrow G^{\prime} \subset E^{\prime}$ is fully $\varphi$-semisolid. Then

(1) $G^{\prime}=E^{\prime}$,

(2) $f$ is $\eta$-quasisymmetric with $\eta=\eta_{\varphi}$,

(3) $f$ is $\psi$-FQC with $\psi=\psi_{\varphi}$.

Proof. By $7.9(3), f$ is $\frac{1}{2}$-locally $\eta$-quasisymmetric with $\eta=\eta_{\varphi}$. Since the domain $G$ is the whole space $E, f$ is $\eta$-quasisymmetric. Since quasisymmetric maps preserve completeness by $6.11, G^{\prime}$ is closed in $E^{\prime}$, and hence $G^{\prime}=E^{\prime}$. Since $f^{-1}: E^{\prime} \rightarrow E$ is $\eta^{\prime}$-quasisymmetric with $\eta^{\prime}(t)=\eta^{-1}\left(t^{-1}\right)^{-1},(3)$ follows from 7.12(4).

7.15. TheOREM. For homeomorphisms $f: E \rightarrow E^{\prime}$, the following conditions are quantitatively equivalent:

(1) $f$ is $\varphi$-FQC,

(2) $f$ is $\eta$-quasisymmetric,

(3) $f$ is weakly $H$-quasisymmetric.

Proof. This follows directly from the part $(1) \Leftrightarrow(4)$ of 7.12 and from 6.7.

7.16. Relations between FQC, quasisymmetry and quasimöbius. Suppose that $f: G \rightarrow$ $G^{\prime}$ is a homeomorphism. If $f$ is FQC, it need not be quasisymmetric or even quasimöbius. But conversely, if $f$ is $\eta$-quasisymmetric, then $f$ is $\varphi$-FQC with $\varphi$-FQC with $\varphi=\varphi_{\eta}$ by 7.12. We next improve this by replacing quasisymmetric by quasimöbius.

7.17. Theorem. Let $G \subset E$ and $G^{\prime} \subset E^{\prime}$ be domains and let $f: G \rightarrow G^{\prime}$ be an $\eta$-quasimöbius homeomorphism. Then $f$ is $\varphi$-FQC with $\varphi=\varphi_{\eta}$.

PRoOF. We shall reduce the situation to the quasisymmetric case by means of auxiliary inversions. Let $D \subsetneq G$ be a domain. By $6.24, f_{D}$ has an extension to a quasimöbius homeomorphism $\bar{f}: \bar{D} \rightarrow \overline{f D}$. By auxiliary translations, we may assume that $0 \in \partial D$, and that $\bar{f}(0)$ is either 0 or $\infty$. Let $u: \dot{E} \rightarrow \dot{E}$ be the inversion $u x=x /|x|^{2}$. If $\bar{f}(0)=0$, we define $g: u D \rightarrow u f D$ by $g(x)=u f u(x)$. If $\bar{f}(0)=\infty$, we define $g: u D \rightarrow f D$ by $g(x)=f u(x)$. Then $g$ is $\eta_{1}$-quasimöbius with $\eta_{1}(t)=81 \eta(81 t)$ by 6.22 . Since $g x \rightarrow \infty$ as $x \rightarrow \infty, g$ is $\eta_{1}$-quasisymmetric. Hence $g$ is $\varphi$-FQC with $\psi=\psi_{\eta}$ by the quasisymmetric version of the theorem. Since $u$ is $36-\mathrm{QH}$ in each subdomain of $E \backslash\{0\}$ by 5.14 , the theorem follows.

7.18. Notes. Fully $\theta$-relative maps in $R^{n}$ were considered in 1963 by F. W. Gehring $\left[\mathrm{Ge}_{2}\right]$. He called these maps briefly $\theta$-mappings and proved that this property is equivalent to quasiconformality. Of the various conditions of Theorems 7.9 and 7.12 , local $q$ quasisymmetry was considered in [I], full coarse quasihyperbolicity in [III], the ring property in [III] and [IV], and the weak local $q$-quasisymmetry was suggested by V. M. Goldshtein and M. Rubin [GR]. 


\section{Mapping problems}

8.1. Summary. We consider the question: Given domains $G$ and $G^{\prime}$, does there exist an FQC map $f: G \rightarrow G^{\prime}$ ? In the positive case, the domains $G$ and $G^{\prime}$ are said to be FQC equivalent. Of course, the same question can be asked replacing FQC by QH, solid or coarsely QH, but the answer is usually the same for each class. One can also ask for estimates for the function $\varphi$ such that $f$ is $\varphi$-FQC.

To give a positive answer we must construct a map $f: G \rightarrow G^{\prime}$. For a negative answer we assume that $f$ exists and obtain a contradiction.

8.2. Whole spaces. We already proved in 7.14 that the whole space $E$ cannot be mapped onto a proper subdomain of $E^{\prime}$ by an FQC map. Hence we consider only homeomorphisms $f: E \rightarrow E^{\prime}$. For these, the properties $\varphi$-FQC, $\eta$-quasisymmetric and weakly $H$-quasisymmetric are quantitatively equivalent by 7.15 . The question arises: When are two given Banach spaces $E$ and $E^{\prime}$ quasisymmetrically equivalent. Very little is known of this problem although there are plenty of interesting results on the corresponding problem for bilipschitz and uniform equivalence; see [Ben], [JLS] and [BL]. Observe that a bilipschitz map $f: E \rightarrow E^{\prime}$ is quasisymmetric and uniformly continuous, but there is no relation between quasisymmetry and uniform continuity.

In particular, the following questions are open:

8.3. Problems. 1. Let $E$ and $E^{\prime}$ be infinite-dimensional separable Banach spaces. Is $E$ quasisymmetrically equivalent to $E^{\prime}$ ?

2. Let $E$ and $E^{\prime}$ be quasisymmetrically equivalent. Are they bilipschitz equivalent?

3. Let $1 \leq p<q<\infty$. Are the spaces $l_{p}$ and $l_{q}$ quasisymmetrically equivalent?

8.4. Spherical rings. For $0<r<s<\infty, t>1$, we set

$$
A(r, s)=B(s) \backslash \bar{B}(r), A(t)=A(1, t) .
$$

These domains and their translates are called spherical rings.

If $\operatorname{dim} E=\infty$, then $E \backslash\{0\}$ is homeomorphic to $E$; see [BP, Cor. 5.1, p. 109]. Hence each spherical ring is homeomorphic to the unit all $B(1)$. We show in 8.5 that such a homeomorphism cannot preserve the class of sets of finite $\mathrm{QH}$ diameter. Hence the map cannot be coarsely QH, let alone FQC.

Two spherical rings in a Banach space can be mapped onto each other by a radial bilipschitz map. However, if the shapes of the rings are very different, the bilipschitz constant becomes large. We consider $\varphi$-FQC maps $f: A(s) \rightarrow A\left(s^{\prime}\right)$ and show that if $s$ and $\varphi$ are given, then $s^{\prime}$ cannot be arbitrarily close to 1 or $\infty$. In fact, we prove in 8.8 the stronger result in which $f$ is only assumed to be $C$-coarsely $M$-QH.

8.5. Theorem. Let $G$ be a spherical ring and let $G^{\prime}$ be a ball. Then there is no homeomorphism $f: G \rightarrow G^{\prime}$ such that for $Q \subset G, k^{\prime}(f Q)<\infty$ if and only if $k(Q)<\infty$. In particular, $f$ cannot be $\mathrm{FQC}$ or coarsely $\mathrm{QH}$.

Proof. It suffices to prove the case where $G=A(1,3)$ and $G^{\prime}=B(1)$. Assume that $f: G \rightarrow G^{\prime}$ is a homeomorphism satisfying the conditions of the theorem. For $S=S(2)$, the components of $G \backslash S$ are $U=A(1,2)$ and $V=A(2,3)$. From 2.7 (or from 2.6) it follows that each pair of points in $S$ can be joined by an $\operatorname{arc} \gamma \subset S$ with $l(\gamma) \leq 8$. Since 
$d(S, \partial G)=1$, this implies that $k(S) \leq 8$, and hence $k^{\prime}(f S)<\infty$. Thus $d\left(f S, \partial G^{\prime}\right)>0$ by $3.12(3)$. Hence there is $t<1$ such that the $\operatorname{ring} A(t, 1)$ does not meet $f S$. Since $A(t, 1)$ is connected, it is contained in one of the components $f U$ and $f V$ of $G^{\prime} \backslash f S$, say in $f V$. Then $f U \subset B(t)$, and hence $k^{\prime}(f U)<\infty$. This gives a contradiction, since $k(U)=\infty$ by $3.12(3)$.

8.6. REMARKS. 1. The idea of the proof above can be used to prove more general results; see [III, 3.13].

2. The proof shows that if $0<r<t<s$, then for $A=A(r, s)$ we have

$$
k_{A}(S(t)) \leq \frac{4 t}{d(S(t), \partial A)} .
$$

Moreover, if $r<t_{1}<t_{2}<s$, the $\mathrm{QH}$ diameter of $A\left(t_{1}, t_{2}\right)$ in $A$ is finite. Indeed, each $x \in A\left(t_{1}, t_{2}\right)$ can be joined to $S\left(t_{2}\right)$ by a radial segment of $\mathrm{QH}$ length less than $\left(t_{2}-t_{1}\right) / \min \left(t_{1}-r, s-t_{2}\right)$. These observations will be useful in the proofs of the next results.

3. In the case $\operatorname{dim} E<\infty$, the reasoning with $\mathrm{QH}$ diameters can be replaced by compactness arguments, and we obtain the well-known topological result that a spherical ring is not homeomorphic to a ball.

8.7. Lemma. Let $f: A(r, s) \rightarrow A\left(r^{\prime}, s^{\prime}\right)$ be a homeomorphism such that a set $Q$ in $A(r, s)$ has a finite $\mathrm{QH}$ diameter if and only if $f Q$ has a finite $\mathrm{QH}$ diameter in $A\left(r^{\prime}, s^{\prime}\right)$. Let $S=S(t)$ with $r<t<s$. Then

(1) $d\left(f S, \partial A^{\prime}\left(r^{\prime}, s^{\prime}\right)\right)>0$,

(2) $A\left(r^{\prime}, s^{\prime}\right) \backslash f S$ has precisely two components $U^{\prime}, V^{\prime}$ with $\partial U^{\prime}=S\left(r^{\prime}\right) \cup f S, \partial V^{\prime}=$ $S\left(s^{\prime}\right) \cup f S$.

Proof. Set $A=A(r, s), A^{\prime}=A\left(r^{\prime}, s^{\prime}\right), k=k_{A}, k^{\prime}=k_{A^{\prime}}$. Then $k(S)<\infty$ by 8.6.2. Hence $k^{\prime}(f S)<\infty$, and (1) follows from 3.12(3).

To prove (2) observe that $A \backslash S$ has precisely two components $U=A(r, t)$ and $V=$ $A(t, s)$. Hence the components of $A^{\prime} \backslash f S$ are $U^{\prime}=f U$ and $V^{\prime}=f V$. By (1) there are numbers $t_{1}, t_{2}$ such that $r^{\prime}<t_{1}<t_{2}<s$ and such that the rings $A\left(r^{\prime}, t_{1}\right)$ and $A\left(t_{2}, s^{\prime}\right)$ do not meet $f S$. If (2) is false, these rings are contained in the same component of $A^{\prime} \backslash f S$, say in $V^{\prime}$. Then $U^{\prime} \subset A\left(t_{1}, t_{2}\right)$, which implies that $k^{\prime}\left(U^{\prime}\right)<\infty$ by 8.6.2. Since $k(U)=\infty$ by $3.13(3)$, this gives a contradiction.

8.8. TheOREM. Suppose that $f: A(s) \rightarrow A\left(s^{\prime}\right)$ is $C$-coarsely $M$-QH. Then $1<s_{1} \leq$ $s^{\prime} \leq s_{2}$, where the numbers $s_{1}$ and $s_{2}$ depend only on $v=(s, M, C)$.

Proof. Set $G=A(s), G^{\prime}=A\left(s^{\prime}\right)$. We first estimate $s^{\prime}$ from below. Set $t=(1+s) / 2$. By 8.7(2), every ray from the origin meets $f S(t)$. Hence we can choose points $x, y \in S(t)$ such that $0 \in[f x, f y]$. For every $z \in G^{\prime}$ we have $\delta^{\prime}(z) \leq\left(s^{\prime}-1\right) / 2$. If $\gamma$ is a rectifiable arc joining $f x$ and $f y$ in $G^{\prime}$, then

$$
l_{k^{\prime}}(\gamma) \geq \frac{2 l(\gamma)}{s^{\prime}-1}>\frac{4}{s^{\prime}-1}
$$


and hence $k^{\prime}(f x, f y) \geq 4 /\left(s^{\prime}-1\right)$. On the other hand, 8.6.2 implies that

$$
k(x, y) \leq k(S) \leq \frac{4(s+1)}{s-1} .
$$

Since $f$ is $C$-coarsely $M$-QH, these inequalities yield

$$
s^{\prime} \geq s_{1}=1+\frac{1}{M(s+1) /(s-1)+C / 4} .
$$

The proof for the upper bound $s^{\prime} \leq s_{2}$ is somewhat harder. Set $a=e^{C+1}$. We may assume that $s^{\prime}>a^{3}$. Let $N \geq 2$ be the unique integer with $a^{N+1}<s^{\prime} \leq a^{N+2}$. It suffices to find an upper bound $N \leq N_{0}(v)$.

For $1 \leq j \leq N$, the spheres $S_{j}^{\prime}=S\left(a^{j}\right)$ lie in $G^{\prime}$, and 8.6.2 gives

$$
k^{\prime}\left(f S_{j}^{\prime}\right) \leq \frac{4 a^{j}}{a^{j}-1}<3 a,
$$

since $a=e^{C+1} \geq e>7 / 3$. Furthermore, if $x \in S_{j}^{\prime}, y \in S_{j+1}^{\prime}$, and $1 \leq j \leq N-1$, then $3.7(1)$ gives

$$
k^{\prime}(x, y) \geq \log \frac{\delta^{\prime}(y)}{\delta^{\prime}(x)}=\log \frac{a^{j+1}-1}{a^{j}-1}>\log a=C+1 .
$$

This gives for the QH distance between $S_{j}^{\prime}$ and $S_{j+1}^{\prime}$ the lower bound

$$
k^{\prime}\left(S_{j}^{\prime}, S_{j+1}^{\prime}\right) \geq C+1 \quad \text { for } 1 \leq j \leq N-1 .
$$

Setting $S_{j}=f^{-1} S_{j}^{\prime}$ we obtain by (8.9)

$$
k\left(S_{j}\right) \leq M k^{\prime}\left(S_{j}^{\prime}\right)+C<3 M a+C
$$

for all $1 \leq j \leq N$. By 3.12(2) this implies

$$
\frac{d\left(S_{j}\right)}{d\left(S_{j}, \partial G\right)} \leq c_{1}=c_{1}(v) .
$$

Since $d\left(S_{j}\right) \geq 2$ by $8.7(2)$, we get

$$
d\left(S_{j}, \partial G\right) \geq 2 / c_{1} \quad \text { for all } 1 \leq j \leq N .
$$

Fix a unit vector $z \in E$ and set $J=[z, s z]$. Then $J \cap S_{j}$ is compact and nonempty for each $j$ by 8.7. Let $u_{j} \in J \cap S_{j}$ be the point with maximal norm. From 8.7 it follows that $\left|u_{j+1}\right|>\left|u_{j}\right|$ for all $1 \leq j \leq N-1$, and hence there is $j \leq N-1$ such that $\left|u_{j+1}-u_{j}\right| \leq(s-1) /(N-1)$. By (8.11) we then have

$$
k\left(u_{j}, u_{j+1}\right) \leq \frac{c_{1}(s-1)}{2(N-1)},
$$

and hence

$$
k^{\prime}\left(f u_{j}, f u_{j+1}\right) \leq \frac{M c_{1}(s-1)}{2(N-1)}+C
$$

Since $k^{\prime}\left(f u_{j}, f u_{j+1}\right) \geq C+1$ by $(8.10)$, we obtain the desired estimate $N \leq N_{0}(v)=$ $1+M c_{1}(s-1) / 2$.

8.12. Balls and half spaces. A domain $H \subset E$ is a half space if there is a bounded nonzero linear functional $A: E \rightarrow \mathbf{R}$ and $\lambda \in \mathbf{R}$ such that $H=\{x \in E: A x<\lambda\}$. In $R^{n}$, each half space is Möbius equivalent to a ball. More generally, this is true in all 
Hilbert spaces. Indeed, if $e \in E$ is a unit vector, the inversion $u x=x /|x|^{2}$ maps the half space $H=\{x: x \cdot e>1\}$ onto the ball $B=B(e / 2,1 / 2)$. The induced homeomorphism $f: H \rightarrow B$ is 4 -QH by 5.14 .

In an arbitrary Banach space, it is not so easy to map a half space onto a ball. Assume that $H \subset E$ is a half space with $H \cap B(1)=\emptyset$. The inversion $u$ maps $H$ onto a domain $D \subset B(1)$, but $D$ is usually not a ball. However, one can show that $D$ is a convex domain, which can be mapped onto a ball by a radial bilipschitz map. Combining these maps we obtain an $M$-QH map of $H$ onto a ball, where $M$ is a universal constant. This was proved with $M=13000$ in [IV, 2.12].

8.13. Towers. We shall construct $\mathrm{QH}$ maps of a half space $H$ onto domains obtained from $H$ by "pulling towers" from $\partial H$. Let $E_{1}$ be a Banach space with $\operatorname{dim} E_{1} \geq 1$. We consider the product space $E=E_{1} \times \mathbf{R}$ as a Banach space with the norm $|(x, t)|=|x|+|t|$. The closed unit ball $\bar{B}(1)$ of $E$ is the suspension of the unit ball $\bar{B}_{1}(1)$ of $E_{1}$ with suspension points $a=(0,1)$ and $-a$. Similarly, the unit sphere $S(1)$ is the suspension of $S_{1}(1)$. Let $P: E \rightarrow E_{1}$ and $Q: E \rightarrow \mathbf{R}$ be the projections, and let $H=\{x: Q x<0\}$ be the lower half space of $E$. Observe that $|x|=|P x|+|Q x|$ for $x \in E$. The set

$$
T=B_{1}(1) \times[0, \infty)=\{x \in E:|P x|<1, Q x \geq 0\}
$$

is the infinite tower with floor $B_{1}(1)$.

Let $G^{\prime}$ be the domain $H \cup T$. Define $f: H \rightarrow G^{\prime}$ by

$$
\begin{cases}f x=\frac{x}{|x|}+a \log \frac{1}{|x|} & \text { for }|x| \leq 1, \\ f x=x & \text { for }|x| \geq 1 .\end{cases}
$$

Then $f$ is a homeomorphism. The half ball $\Delta=B(1) \cap H$ is the basement of the tower $T$, and $f$ maps $\Delta$ onto $T \cup \Delta$ carrying each hemisphere $F(t)=S(t) \cap H, 0<t<1$ by a similarity map onto $F(1)-a \log t$. See Figure 2 , where $F(1)$ is indicated by a thick line.

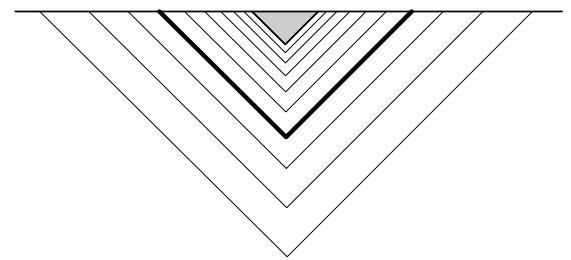

Half space

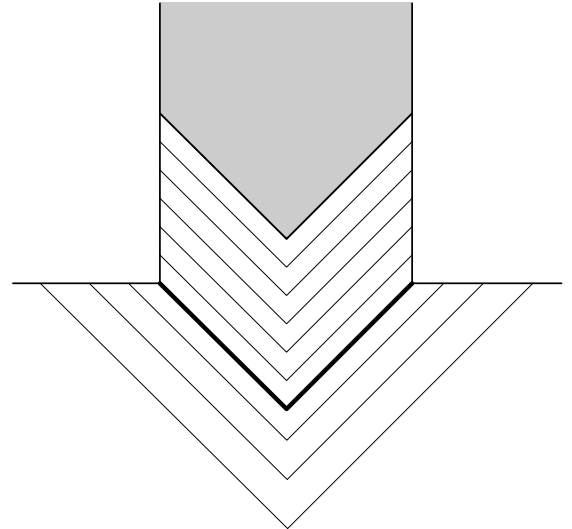

Infinite tower

Fig. 2 
To obtain an expression for the inverse map $g=f^{-1}: G^{\prime} \rightarrow H$ we write $R y=$ $Q y+1-|P y|$ for $y \in T \cup \Delta$. Geometrically, $R y$ is the vertical distance between $y$ and the floor $F(1)$ of the basement. If $x \in \Delta$ and $y=f x$, we have $R y=-\log |x|$. Thus $|x|=e^{-R y}$ and

$$
g y=e^{-R y}(y-a R y) .
$$

We show that $f: H \rightarrow G^{\prime}$ is 6-QH. By 5.6 it suffices to show that $L_{k}(x, t) \leq 6$ and $L_{k}(y, g) \leq 6$ for all $x \in H$ and $y \in G^{\prime}$. Let $x \in H$. If $|x|>1$, then $L(x, f)=$ $1, \delta(x)=|Q x|, \delta^{\prime}(f x) \geq|Q x|$, and hence $L_{k}(x, f) \leq 1$ by 5.4. Assume that $|x|<1$. Since $L(x, N)=1$ for the norm function $N x=|x|$, the $L$-formulas 5.3(3) give

$$
L(x, f) \leq \frac{1}{|x|}+\frac{|x|}{|x|^{2}}+\frac{|a|}{|x|}=\frac{3}{|x|} .
$$

Since $\delta(x)=|Q x|$ and $\delta^{\prime}(f x) \geq 1-|P f x|=|Q x| /|x|$, this implies

$$
L_{k}(x, f) \leq 3 \text {. }
$$

This is obviously true also in the case $|x|=1$ and hence for all $x \in H$.

Next assume that $y=f x \in G^{\prime}$. If $y \notin T \cup \bar{\Delta}$, then $L(y, g)=1, \delta^{\prime}(y) \leq 2|Q y|$ and $\delta(g y)=\delta(y)=|Q y|$. Hence $L_{k}(y, g) \leq 2$. Assume that $y \in T \cup \Delta$. For every $y^{\prime} \in T \cup \Delta$ we have

$$
\left|R y-R y^{\prime}\right|=\left|Q y-Q y^{\prime}-\right| P y|+| P y^{\prime}|| \leq\left|Q y-Q y^{\prime}\right|+\left|P y-P y^{\prime}\right|=\left|y-y^{\prime}\right|,
$$

and hence $L(y, R) \leq 1$. Consequently, (1) and 5.3(3) give $L(y, g) \leq e^{-R y} L(y, R) \mid y-$ $a R y\left|+e^{-R y}(1+|a| L(y, R)) \leq 3 e^{-R y}=3\right| x \mid$, since $y-a R y=x /|x|$ for $x=g y$. This holds also for $y \in H \cap \partial \Delta$. Since $\delta(g y)=|Q x|$ and $\delta^{\prime}(y) \leq 2(1-|P y|)=2|Q x| /|x|$, this implies

$$
L_{k}(y, g) \leq 6
$$

and we have proved that $f: H \rightarrow G^{\prime}$ is $6-\mathrm{QH}$.

For $\lambda>0$, the set

$$
T_{\lambda}=B_{1}(1) \times[0, \lambda)=\{x \in T: Q x<\lambda\}
$$

is a finite tower of height $\lambda$ with floor $B_{1}(1)$. We obtain a homeomorphism $f_{1}: H \rightarrow H \cup T_{\lambda}$ by setting $f_{1} x=f x$ for $|x| \geq e^{-\lambda}$ and $f_{1} x=e^{\lambda} x+\lambda a$ for $|x| \leq e^{-\lambda}$. Thus $f_{1} \mid H \cap B\left(e^{-\lambda}\right)$ is a similarity. It is an easy exercise to verify that (2) and (3) hold also for the map $f_{1}$, and hence it is $6-\mathrm{QH}$.

We can use these maps to build QH maps of the half space $H$ onto domains with several towers. Let $\mathcal{A}$ be a family of disjoint open balls in $E_{1}$. On each $B=B_{1}\left(z_{B}, r_{B}\right) \in \mathcal{A}$ we erect a tower $T_{B}$ with floor $B$. This tower can be finite $B \times\left[0, \lambda_{B}\right)$ or infinite $B \times[0, \infty)$. Each $T_{B}$ has a basement $\Delta_{B}=B\left(z_{B}, r_{B}\right) \cap H$.

The union of $H$ and all towers $T_{B}, B \in \mathcal{A}$ is a domain $G^{\prime}$, called an organ pipe domain; see Figure 3. We map each $\Delta_{B}$ onto $T_{B} \cup \Delta_{B}$ by a copy of the map $f$ or $f_{1}$ described above. Setting $F x=x$ for $x \in H \backslash \cup\left\{\Delta_{B}: B \in \mathcal{A}\right\}$ we obtain a homeomorphism $F: H \rightarrow G^{\prime}$. The estimates (2) and (3) are valid for $F$, and hence $F$ is 6 -QH. 


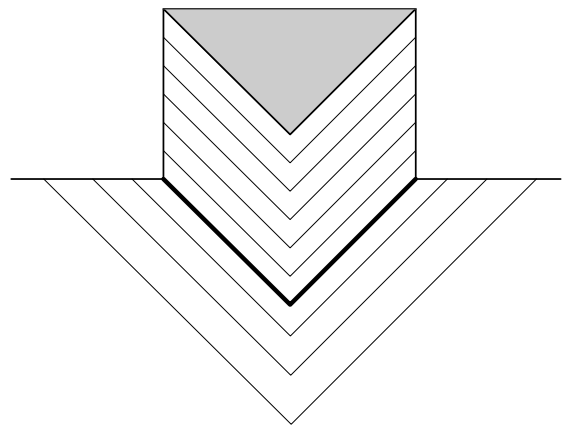

Finite tower

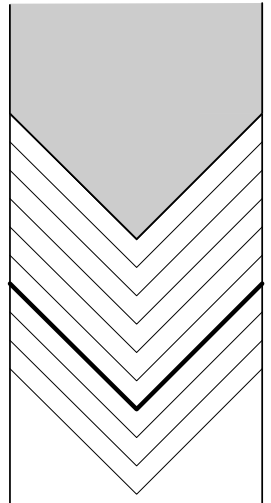

Tube

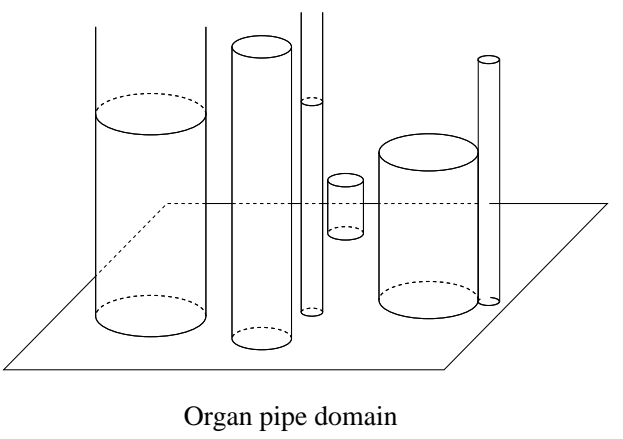

Fig. 3

8.14. Tubes. Let $H \subset E=E_{1} \times \mathbf{R}$ be as in 8.13. Setting

$$
f x=\frac{x}{|x|}+a \log \frac{1}{|x|}
$$

for all $x \in H$, we obtain a homeomorphism $f$ of $H$ onto the infinite tube $Z=B_{1}(1) \times \mathbf{R}=$ $\{x \in E:|P x|<1\}$. Since $d(y, \partial Z)=1-|P y|$ for all $y \in Z$, the estimates in 8.13 show that $f: H \rightarrow Z$ is $3-\mathrm{QH}$.

8.15. Broken tubes. In examples 8.13 and 8.14, the dimension of $E$ plays no role, and it can be finite or infinite. We next give an example where it is essential that $\operatorname{dim} E=\infty$. Let $E$ be an infinite-dimensional separable Hilbert space, and let $\left(e_{j}\right)_{j \in \mathbf{Z}}$ be an orthonormal family in $E$, indexed by the set $\mathbf{Z}$ of all integers. Let $\gamma$ be the line spanned by $e_{0}$, and set $a_{j}=j e_{0} \sqrt{2}$ for $j \in \mathbf{Z}$. Let $g_{0}: \gamma \rightarrow E$ be the map for which $g_{0} a_{j}=e_{j}$ for all $j \in \mathbf{Z}$ and which is affine on each line segment $\gamma_{j}=\left[a_{j-1}, a_{j}\right]$. Thus $g_{0} \mid \gamma_{j}$ is an isometry onto $\gamma_{j}^{\prime}=\left[e_{j-1}, e_{j}\right]$. Let $T$ be the tubular neighborhood $\{x \in E: d(x, \gamma)<1 / 10\}$ of $\gamma$. It is not difficult to extend $g_{0}$ to a homeomorphism $g$ of $T$ onto a neighborhood $T^{\prime}$ of $\gamma^{\prime}$ such that $g$ is locally $M$-bilipschitz with a universal constant $M$; the details are left to the reader. By 5.16, $g$ is $M^{2}$-QH. From 8.14 it follows that there is a 3 -QH map $f: H \rightarrow T$ if $H=\left\{x: x \cdot e_{0}<0\right\}$ and $T$ are considered with the norm $N x=|P x|+|Q x|$ of 8.13 . 
Since $|x|^{2}=|P x|^{2}+|Q x|^{2}$, we have $|x| \leq N x \leq|x| \sqrt{2}$ for all $x \in E$. By 5.16 , the identity maps of $H$ and $T$ are $2-\mathrm{QH}$ with respect to the norms $|x|$ and $|N x|$. It follows that the map $h=g f: H \rightarrow T^{\prime}$ is $M_{1}-\mathrm{QH}$ in the original norm with $M_{1}=12 M^{2}$.

We say that the domain $T^{\prime}$ is a broken tube with core $\gamma^{\prime}$. It has several interesting properties. For example, it contains the connected set $\gamma^{\prime}$, which has finite relative size $d\left(\gamma^{\prime}\right) / d\left(\gamma^{\prime}, \partial T^{\prime}\right)$ but infinite $\mathrm{QH}$ diameter. This phenomenon is impossible in $R^{n}$; see $\left[\mathrm{TV}_{2}, 6.9\right]$. It is also impossible for uniform domains in any Banach spaces (Section 10).

The pre-image $\alpha=h^{-1} \gamma^{\prime}$ is the ray $\alpha=\left\{t e_{0}: t<0\right\}$, and $g$ defines a homeomorphism $h_{D}$ of $D=H \backslash \alpha$ onto $D^{\prime}=T^{\prime} \backslash \gamma^{\prime}$. Since $h$ is $M_{1}-\mathrm{QH}, h_{D}$ is $M_{2}-\mathrm{QH}$ with $M_{2}=4 M_{1}^{2}$ by 5.12. Observe that $\partial D=\partial H \cup \alpha$ is connected while $\partial D^{\prime}=\partial T^{\prime} \cup \gamma^{\prime}$ has two components $\partial T^{\prime}$ and $\gamma^{\prime}$. In $\dot{R}^{n}$, the number of the boundary components of a domain is a topological invariant, but we see that in arbitrary Banach spaces it is not even a QH invariant. However, in this example $\partial D$ is not very "firmly" connected, since it becomes disconnected if the points 0 and $\infty$ are removed from it. The following question is open:

8.16. Problem. Does there exist a solid map of a ball onto a domain with nonconnected boundary?

8.17. ExERCISE. Show that the map $g_{0}: \gamma \rightarrow \gamma^{\prime}$ in 8.15 has the following properties:

(1) $g_{0}$ is weakly 2-quasisymmetric,

(2) $g_{0}^{-1}$ is not weakly quasisymmetric,

(3) $g_{0}$ is not quasisymmetric.

8.18. Notes. In $R^{n}$, the estimates $s_{1} \leq s^{\prime} \leq s_{2}$ of 8.8 for $K$-quasiconformal maps are usually proved using the moduli of the path families joining the boundary components of the rings. Another method is based on the isoperimetric inequality. These give the sharp estimates

$$
K^{-1} \log s \leq \log s^{\prime} \leq K \log s
$$

see [Kü, Satz 1]. This is the only result known to the author in the theory of quasiconformal maps in $R^{n}$ which allows a dimension-free conclusion in terms of the metric dilatation defined in 1.1 .

The towers, tubes, and organ pipe domains in $R^{3}$ were considered by F. W. Gehring and the author $[\mathrm{GV}]$ in 1965.

\section{Neargeodesics and coarse length}

9.1. Summary. We develop some tools that are needed in a deeper study of the free quasiworld. We found in 3.5 that a pair of points in a domain $G \subsetneq E$ cannot always be connected by a $\mathrm{QH}$ geodesic. However, we show that they can be joined by a neargeodesic, that is, by an arc that is quasiconvex in the $\mathrm{QH}$ metric.

Since an FQC map $f: G \rightarrow G^{\prime}$ may map a rectifiable arc $\gamma \subset G$ onto a nonrectifiable arc, there is no use to compare the lengths of $\gamma$ and $f \gamma$. We therefore introduce for each $h>0$ a finite number $l(\gamma, h)$, called the $h$-coarse length of $\gamma$, and the corresponding concept of coarse quasiconvexity. Arcs that are coarsely quasiconvex in the QH metric turn out to be particularly important, and they are called solid arcs. We show that this 
property is preserved by FQC maps and, more generally, by coarsely QH maps. This result will be useful in Section 11 where we study maps of uniform domains.

9.2. Neargeodesics. Let $\gamma$ be an arc in a domain $G \subsetneq E$ and let $c \geq 1$. We say that $\gamma$ is a $c$-neargeodesic in $G$ if $\gamma$ is $c$-quasiconvex in the $\mathrm{QH}$ metric of $G$. In other words,

$$
l_{k}(\gamma[x, y]) \leq c k(x, y) \quad \text { for all } x, y \in \gamma .
$$

For $c=1$ this means that $\gamma$ is a $\mathrm{QH}$ geodesic.

9.3. LemmA. Suppose that $a \in G \subsetneq E$, that $0<t<1$, and that $\gamma$ is a rectifiable arc in $\bar{B}(a, t \delta(a))$. Then

$$
\frac{1}{1+t} \leq \frac{l_{k}(\gamma) \delta(a)}{l(\gamma)} \leq \frac{1}{1-t}
$$

Moreover, if $t \leq 1 / 2$, then every line segment in $\bar{B}(a, t \delta(a))$ is a c-neargeodesic in $G$ with $c=(1+2 t)^{2} \leq 4$.

Proof. Since $\delta(a)(1-t) \leq \delta(x) \leq \delta(a)(1+t)$ for all $x \in \bar{B}(a, t \delta(a))$, the inequalities follow by integration along $\gamma$. Suppose that $t \leq 1 / 2$ and that $\gamma=[x, y] \subset \bar{B}(a, t \delta(a))$. Since $1 /(1-t) \leq 1+2 t$, the second inequality of the lemma and 3.7(4) imply

$$
l_{k}(\gamma) \leq(1+2 t)|x-y| / \delta(a) \leq(1+2 t)^{2} k(x, y) .
$$

9.4. TheOREm. Let $a, b \in G \subsetneq E$ and let $c>1$. Then there is a c-neargeodesic from a to $b$ in $G$.

ProOF. The proof is somewhat long, but its idea is simple: We join $a$ and $b$ by an arc $\gamma$ with $l_{k}(\gamma)$ very close to $k(a, b)$, and then choose a suitable inscribed polygon.

For $q>0$ we write $c_{1}=c_{1}(q)=(1+2 q)^{2}$. Choose $q_{0}>0$ such that

$$
q_{0} \leq k(a, b) / 10, c_{1}\left(q_{0}\right)<5 / 4 .
$$

Then $q_{0}<1 / 8$. We shall prove the theorem by constructing for every $q \leq q_{0}$ an arc $\beta$ joining $a$ and $b$ such that $\beta$ is a $c(q)$-neargeodesic with $c(q) \rightarrow 1$ as $q \rightarrow 0$.

Let $0<q \leq q_{0}$. Choose an arc $\gamma$ from $a$ to $b$ with $l_{k}(\gamma) \leq k(a, b)+q^{2}$. Then

$$
l_{k}(\gamma[x, y]) \leq k(x, y)+q^{2}
$$

for all $x, y \in \gamma$, since assuming $x \in \gamma[a, y]$ we have

$$
k(a, x)+l_{k}(\gamma[x, y])+k(y, b) \leq l_{k}(\gamma) \leq k(a, x)+k(x, y)+k(y, b)+q^{2} .
$$

Since $q \leq k(a, b) / 10$, there is $\lambda=\lambda(q)$ such that

$$
q / 4 \leq \lambda \leq q / 2, l_{k}(\gamma)=m \lambda
$$

for some integer $m \geq 1$. We divide $\gamma$ by successive points $a=x_{0}, \ldots, x_{m}=b$ into subarcs $\gamma_{i}=\gamma\left[x_{i-1}, x_{i}\right]$ with $l_{k}\left(\gamma_{i}\right)=\lambda$. We show that the union $\beta$ of the line segments $\beta_{i}=\left[x_{i-1}, x_{i}\right]$ is the desired arc.

Assume that $x, y \in \beta, x \neq y$. Although we have not yet shown that $\beta$ is an arc, the QH length $l_{k}(\beta[x, y])$ is defined in the obvious way as soon as we fix $i$ and $j$ such that $x \in \beta_{i}, y \in \beta_{j}$. It suffices to find $c=c(q)$ such that $c(q) \rightarrow 1$ as $q \rightarrow 0$ and such that

$$
l_{k}(\beta[x, y]) \leq c k(x, y) .
$$


We consider four cases.

Case 1. There is $i$ such that $x, y \in \beta_{i}$. Since $k\left(x_{i-1}, x_{i}\right) \leq l_{k}\left(\gamma_{i}\right)=\lambda \leq q / 2<1$, Theorem 3.9 gives $\left|x_{i-1}-x_{i}\right| \leq q \delta\left(x_{i}\right)$. Hence (9.7) follows from 9.3 with $c=c_{1}$.

Case 2. $x$ and $y$ are vertices, say $x=x_{i}, y=x_{i+s}, s \geq 1$. Applying Case 1 to the line segments between $x$ and $y$ we get

$$
\begin{aligned}
l_{k}(\beta[x, y]) & =\sum_{j=1}^{s} l_{k}\left(\beta_{i+j}\right) \leq c_{1} \sum_{j=1}^{s} k\left(x_{i+j-1}, x_{i+j}\right) \leq c_{1} \sum_{j=1}^{s} l_{k}\left(\gamma_{i+j}\right) \\
& =c_{1} l_{k}(\gamma[x, y]) .
\end{aligned}
$$

On the other hand, (9.6) gives

$$
k(x, y) \geq l_{k}(\gamma[x, y])-q^{2}=s \lambda-q^{2} \geq q / 4-q^{2}>q / 8,
$$

since $q<1 / 8$. Together with (9.6) and (9.8), this implies (9.7) with $c=c_{2}=c_{1}+8 c_{1} q$.

Case 3. There are $i \geq 1$ and $j \geq i+2$ such that $x \in \beta_{i}, y \in \beta_{j}$. By means of Case 2 we obtain

$$
\begin{aligned}
l_{k}(\beta[x, y]) & =l_{k}\left(\beta\left[x_{i-1}, x_{j}\right]\right)-l_{k}\left(\beta\left[x_{i-1}, x\right]\right)-l_{k}\left(\beta\left[y, x_{j}\right]\right) \\
& \leq c_{2} k\left(x_{i-1}, x_{j}\right)-k\left(x_{i-1}, x\right)-k\left(y, x_{j}\right) \\
& \leq c_{2} k(x, y)+\left(c_{2}-1\right)\left[k\left(x_{i-1}, x\right)+k\left(y, x_{j}\right)\right] .
\end{aligned}
$$

Here

$$
k\left(x_{i-1}, x\right) \leq l_{k}\left(\beta_{i}\right) \leq c_{1} k\left(x_{i-1}, x_{i}\right) \leq c_{1} \lambda \leq c_{1} q / 2,
$$

and similarly $k\left(y, x_{j}\right) \leq c_{1} q / 2$. By (9.6), these estimates yield

$$
\begin{aligned}
k(x, y) & \geq k\left(x_{i-1}, x_{j}\right)-k\left(x_{i-1}, x\right)-k\left(y, x_{j}\right) \\
& \geq l_{k}\left(\gamma\left[x_{i-1}, x_{j}\right]\right)-q^{2}-2 c_{1} \lambda \geq\left(3-2 c_{1}\right) \lambda-q^{2} \geq\left(5-4 c_{1}\right) q / 8,
\end{aligned}
$$

where we also made use of the inequalities $\lambda \geq q / 4$ and $q \leq 1 / 8$. By (9.5), the right-hand side is positive. Combining these estimates we obtain (9.7) with

$$
c=c_{3}(q)=c_{2}+\frac{8 c_{1}\left(c_{2}-1\right)}{5-4 c_{1}} .
$$

Case 4. There is $i$ such that $x \in \beta_{i}$ and $y \in \beta_{i+1}$. If $x=x_{i-1}$ or $y=x_{i+1}$, we are in Case 3 . The general case is reduced to these special cases as follows:

We may assume that $x \neq x_{i} \neq y$. For $K \geq 1$ let $g: E \rightarrow E$ be the similarity defined by $g u=x_{i}+K\left(u-x_{i}\right)$. We can choose $K$ such that $g x \in \beta_{i}, g y \in \beta_{i+1}$, and either $g x=x_{i-1}$ or $g y=x_{i+1}$. In Case 1 we showed that $\beta_{i} \cup \beta_{i+1} \subset \bar{B}\left(x_{i}, q \delta\left(x_{i}\right)\right)$. Applying 9.3 twice we obtain

$$
l_{k}(\beta[x, y]) \leq \frac{l(\beta[x, y])}{(1-q) \delta\left(x_{i}\right)}=\frac{l(\beta[g x, g y])}{K(1-q) \delta\left(x_{i}\right)} \leq \frac{(1+q) l_{k}(\beta[g x, g y])}{K(1-q)} .
$$

By Case 3 we have $l_{k}(\beta[g x, g y]) \leq c_{3} k(g x, g y)$. These estimates and 3.7 yield

$$
\begin{aligned}
l_{k}(\beta[x, y]) & \leq \frac{(1+q) c_{3}|g x-g y|}{K(1-q)^{2} \delta\left(x_{i}\right)}=\frac{(1+q) c_{3}|x-y|}{(1-q)^{2} \delta\left(x_{i}\right)} \leq \frac{(1+q)(1+2 q) c_{3} k(x, y)}{(1-q)^{2}} \\
& =c_{4} k(x, y)
\end{aligned}
$$

where $c_{4}=c_{4}(q) \rightarrow 1$ as $q \rightarrow 0$. 
9.9. Coarseness. The intuitive idea behind coarseness is that we fix a number $h \geq 0$ and look at a metric space $X$ with $h$-coarse eyes, which means that we cannot distinguish between points $x, y$ with $|x-y|<h$. One should observe, however, that this is not an equivalence relation. If $h=0$, there is no coarseness.

For example, in the coarse Lipschitz condition $|f x-f y| \leq M|x-y|+C$ of 2.2 , the constant $C$ plays the role of $h$; the map looks $M$-Lipschitz if we only see $C$-coarsely where $f x$ is.

9.10. Coarse length. Let $\gamma$ be an arc in a metric space $X$ and let $h \geq 0$. We consider finite sequences $\bar{x}=\left(x_{0}, \ldots, x_{m}\right), m \geq 1$, of successive points of $\gamma$. We say that $\bar{x}$ is an $h$-coarse sequence if $\left|x_{j-1}-x_{j}\right| \geq h$ for all $1 \leq j \leq m$. We write

$$
s(\bar{x})=\sum_{j=1}^{m}\left|x_{j-1}-x_{j}\right|
$$

and define the $h$-coarse length of $\gamma$ as

$$
l(\gamma, h)=\sup s(\bar{x})
$$

over all $h$-coarse sequences $\bar{x}$ on $\gamma$. We agree that $\sup \varnothing=0$, and hence $l(\gamma, h)=0$ whenever $d(\gamma)<h$. This is natural, since our $h$-coarse eyes see $\gamma$ as a single point.

Observe that $l(\gamma, 0)$ is the ordinary length $l(\gamma)$, and it may be infinite. If $h>0$, then $l(\gamma, h)<\infty$ by compactness. We list some obvious properties of the coarse length.

9.11. TheOrem. Let $\gamma$ be an arc in a metric space and let $h \geq 0$.

(1) The function $h \mapsto l(\gamma, h)$ is decreasing.

(2) $d(\gamma) \leq h \vee l(\gamma, h)$.

(3) $\gamma^{\prime} \subset \gamma$ implies $l\left(\gamma^{\prime}, h\right) \leq l(\gamma, h)$.

(4) $l(\gamma, h)=\sup s(\bar{x})$ over all $\bar{x}=\left(x_{0}, \ldots, x_{m}\right)$ such that $h \leq\left|x_{j-1}-x_{j}\right| \leq 2 h$ for $1 \leq j \leq m$.

9.12. Coarse quasiconvexity. Let $h \geq 0$ and $c \geq 1$. We say that a metric space $X$ is $h$-coarsely c-quasiconvex if each pair of points $a, b \in X$ can be joined by an $\operatorname{arc} \gamma \subset X$ such that $l(\gamma, h) \leq c|a-b|$. In particular, an arc $\gamma$ is $h$-coarsely $c$-quasiconvex if

$$
l(\gamma[x, y], h) \leq c|x-y| \quad \text { for all } x, y \in \gamma .
$$

Recall from 2.2 that a map $f: X \rightarrow Y$ is $C$-coarsely $M$-bilipschitz if

$$
(|x-y|-C) / M \leq|f x-f y| \leq M|x-y|+C \quad \text { for all } x, y \in X .
$$

If only the second inequality is assumed, then $f$ is $C$-coarsely $M$-Lipschitz.

9.13. Theorem. Suppose that $X$ is h-coarsely c-quasiconvex and that $f: X \rightarrow Y$ is $C$-coarsely $M$-bilipschitz. Then $f X$ is $h_{1}$-coarsely $c_{1}$-quasiconvex with

$$
h_{1}=M(h \vee 2 c C)+C, c_{1}=2 c M(M+1) .
$$

Proof. Let $a, b \in X$ and choose an arc $\gamma \subset X$ joining $a$ and $b$ such that

$$
l(\gamma, h) \leq c|a-b| .
$$


It suffices to show that

$$
l\left(f \gamma, h_{1}\right) \leq c_{1}|f a-f b| .
$$

Setting $h^{\prime}=M(h \vee C)+C$ we first show that

$$
l\left(f \gamma, h^{\prime}\right) \leq(M+1) l(\gamma, h) .
$$

Let $\bar{y}=\left(y_{0}, \ldots, y_{m}\right)$ be an $h^{\prime}$-coarse sequence on $f \gamma$. Writing $\bar{x}=\left(x_{0}, \ldots, x_{m}\right)$ with $x_{j}=f^{-1} y_{j}$ we have

$$
\left|x_{j-1}-x_{j}\right| \geq\left(\left|y_{j-1}-y_{j}\right|-C\right) / M \geq\left(h^{\prime}-C\right) / M=h \vee C .
$$

Hence $\bar{x}$ is $h$-coarse, which implies that $s(\bar{x}) \leq l(\gamma, h)$. Moreover, $\left|x_{j-1}-x_{j}\right| \geq C$, and we obtain

$$
s(\bar{y}) \leq \sum_{j=1}^{m}\left(M\left|x_{j-1}-x_{j}\right|+C\right) \leq(M+1) s(\bar{x}) \leq(M+1) l(\gamma, h) .
$$

This implies (9.16).

To prove (9.15) we first assume that $|a-b| \geq 2 C$. Then

$$
|f a-f b| \geq(|a-b|-C) / M \geq|a-b| / 2 M .
$$

Since $h^{\prime} \leq h_{1}$, this, (9.14) and (9.16) yield

$$
l\left(f \gamma, h_{1}\right) \leq(M+1) c|a-b| \leq c_{1}|f a-f b|,
$$

which is (9.15).

Next assume that $|a-b|<2 C$. Let $x, y \in \gamma$. It suffices to show that $|f x-f y|<h_{1}$, since this will imply that $l\left(f \gamma, h_{1}\right)=0$, and (9.15) holds trivially. If $|x-y|<h$, then $|f x-f y|<M h+C \leq h_{1}$. If $|x-y| \geq h$, then the pair $(x, y)$ is a $h$-coarse sequence, and hence

$$
|x-y| \leq l(\gamma, h) \leq c|a-b|<2 c C,
$$

which implies that

$$
|f x-f y|<2 M c C+C \leq h_{1}
$$

9.17. Solid arcs. Suppose that $G \subsetneq E$ is a domain. For $h \geq 0$, we let $l_{k}(\gamma, h)$ denote the $h$-coarse length of an arc $\gamma \subset G$ in the $\mathrm{QH}$ metric of $G$. For $c \geq 1$, we say that $\gamma$ is a $(c, h)$-solid arc if $\gamma$ is $h$-coarsely $c$-quasiconvex in the $\mathrm{QH}$ metric. In other words,

$$
l_{k}(\gamma[x, y], h) \leq c k(x, y) .
$$

Thus $\gamma$ is $(c, 0)$-solid if and only if $\gamma$ is a $c$-neargeodesic.

The following result is a special case of 9.13. It is very useful in the study of maps of uniform domains (Section 11). In many cases, it acts as a substitute for the method of moduli of path families, which is useless in the free quasiworld.

9.18. Solid Arc Theorem. Suppose that $f: G \rightarrow G^{\prime}$ is $C$-coarsely $M$-QH and that $\gamma$ is a $(c, h)$-solid arc in $G$. Then $f \gamma$ is a $\left(c_{1}, h_{1}\right)$-solid arc in $G^{\prime}$ with

$$
h_{1}=M(h \vee 2 c C)+C, c_{1}=2 c M(M+1) .
$$

9.19. Notes. This section is from [II]. 


\section{Uniform domains}

10.1. SummarY. Roughly speaking, a domain in $E$ is uniform if one can travel from one point to another without going very far from these points or very close to the boundary of the domain. Half spaces, balls and spherical rings are uniform while an infinite tube is not. Uniform domains are extremely useful in the free quasiworld, since FQC maps between them have several pleasant properties, which will be considered in Section 11.

We give two approaches to uniform domains. One is based on the QH metric and the other on so-called cigars. We prove that they give quantitatively the same concept, and give some examples and properties of uniform domains.

10.2. Definitions. Let $G \subsetneq E$ be a domain. We recall from 3.7(1) the inequality $j_{G} \leq k_{G}$, where

$$
j_{G}(x, y)=\log \left(1+\frac{|x-y|}{\delta(x) \wedge \delta(y)}\right) .
$$

Let $c \geq 1$. We say that $G$ is a quasihyperbolically $c$-uniform domain if $k_{G}(x, y) \leq c j_{G}(x, y)$ for all $x, y \in G$.

Let $\gamma$ be an arc in $G$ with endpoints $a, b$. For $x \in \gamma$ we set

$$
\varrho_{d}(x)=d(\gamma[a, x]) \wedge d(\gamma[x, b]) .
$$

If $\gamma$ is rectifiable, we also write

$$
\varrho_{l}(x)=l(\gamma[a, x]) \wedge l(\gamma[x, b]) .
$$

For $c \geq 1$, we define the diameter $c$-cigar $\operatorname{cig}_{d}(\gamma, c)$ and the length $c$-cigar $\operatorname{cig}_{l}(\gamma, c)$ by

$$
\begin{aligned}
& \operatorname{cig}_{d}(\gamma, c)=\cup\left\{B\left(x, \varrho_{d}(x) / c\right): x \in \gamma \backslash\{a, b\}\right\}, \\
& \operatorname{cig}_{l}(\gamma, c)=\cup\left\{B\left(x, \varrho_{l}(x) / c\right): x \in \gamma \backslash\{a, b\}\right\} .
\end{aligned}
$$

The length cigar is only defined if $\gamma$ is rectifiable. Observe that these sets are open neighborhoods of $\gamma \backslash\{a, b\}$ and that $\operatorname{cig}_{d}(\gamma, c) \subset \operatorname{cig}_{l}(\gamma, c)$.

For $c \geq 1$ we say that $G$ is a $c$-uniform domain if each pair of points $a, b \in G$ can be joined by a rectifiable arc $\gamma \subset G$ such that the following uniformity conditions hold:

(1) $\operatorname{cig}_{l}(\gamma, c) \subset G$,

(2) $l(\gamma) \leq c|a-b|$.

Observe that (1) can also be written as

(1) $\varrho_{l}(x) \leq c \delta(x)$

for all $x \in \gamma$. Condition (1) is called the cigar condition, and (2) is the turning condition.

10.3. REMARKS. If we replace (1) and (2) by

$\left(1^{\prime}\right) \operatorname{cig}_{d}(\gamma, c) \subset G$,

$\left(2^{\prime}\right) d(\gamma) \leq c|a-b|$

we get a concept which in the case $E=R^{n}$ is $n$-quantitatively equivalent to $c$-uniformity. In an arbitrary Banach space, however, this leads to a different property, which does not seem to be very useful. For example, in the broken tube of 8.15 one can join points by 
arcs satisfying $\left(1^{\prime}\right)$ and $\left(2^{\prime}\right)$, but it is not a uniform domain. Nevertheless, the diameter cigars are useful also in the free quasiworld.

A third possibility in $R^{n}$ is to use distance cigars, based on the function $\varrho(x)=$ $|x-a| \wedge|x-b|$.

10.4. ExAmples. 1. We first show that each ball $B \subset E$ is 2 -uniform. In fact, we prove the stronger result that each pair of points $a, b \in \bar{B}, a \neq b$, can be joined by an arc $\gamma$ satisfying the uniformity conditions in $B$. We may assume that $B$ is the unit ball $B(1)$. Set $z=(a+b) / 2$. We consider two cases.

Case 1. $|z| \geq|a-b| / 2$. Let $y \in[0, z]$ be the point with $|y-z|=|a-b| / 2$ and write $\gamma=[a, z] \cup[z, b]$. Then

$$
|a-y| \leq|a-z|+|z-y|=|a-b|,
$$

and similarly $|b-y| \leq|a-b|$. Hence $\gamma$ satisfies the turning condition $l(\gamma)=|a-y|+|b-y| \leq$ $2|a-b|$.

To prove the cigar condition it suffices to consider a point $x \in[a, y]$ and show that $\varrho_{l}(x) \leq 2 \delta(x)$. Writing $x=(1-t) a+t y$ with $0 \leq t \leq 1$ we have

$$
\delta(x)=1-|x| \geq 1-(1-t)|a|-t|y| \geq 1-(1-t)-t(1-|y-z|)=t|a-b| / 2 .
$$

Hence

$$
\varrho_{l}(x) \leq|a-x|=t|a-y| \leq t|a-b| \leq 2 \delta(x) .
$$

Case 2. $|z| \leq|a-b| / 2$. We show that $\gamma=[a, 0] \cup[0, b]$ satisfies the uniformity conditions. We have

$$
|a| \leq|a-z|+|z| \leq|a-b|,
$$

and similarly $|b| \leq|a-b|$. Hence $\gamma$ satisfies the turning condition $l(\gamma)=|a|+|b| \leq 2|a-b|$. If $x \in[a, 0]$, then

$$
\varrho_{l}(x) \leq|a-x|=|a|-|x| \leq 1-|x|=\delta(x),
$$

and hence the cigar condition holds with the constant 1.

2. More generally, every bounded convex domain $G \subset E$ is uniform. If $B(r) \subset G \subset$ $B(b r)$, then $G$ is $c$-uniform with $c=c(b)$. This was proved in [Al] by explicit constructions similar to those in Example 1. Alternatively, one can make use of a radial bilipschitz map of $G$ onto a ball.

3. Let $H \subset E$ be a half space. We show that if $c>2$, each pair of points $a, b \in$ $\bar{H} \backslash\{\infty\}, a \neq b$, can be joined by an arc $\gamma$ satisfying the uniformity conditions in $H$. This will imply that $H$ is $c$-uniform for all $c>2$.

We may assume that $0 \in \partial H$. Set $z=(a+b) / 2$. If $z \in H$, we choose a point $w \in \partial H$ such that $|z-w| \leq c \delta(z) / 2$. Set $y=z+r(z-w)$ where $r$ is chosen to that

$$
|y-z|=|a-b| / 2 \text {. }
$$

Then

$$
\delta(y)=|y-w| \delta(z) /|z-w| \geq 2|y-z| / c=|a-b| / c .
$$

If $z \in \partial H$ we apply the well known Riesz lemma [CC, p. 68] to find $y \in H$ such that (a) and $(\mathrm{b})$ are true. We show that $\gamma=[a, y] \cup[y, b]$ is the desired arc. 
Since $|a-y| \leq|a-z|+|z-y|=|a-b|$, and similarly $|b-y| \leq|c-b|$, the turning condition $l(\gamma)=|a-y|+|b-y| \leq 2|a-b|$ is true. We show that the cigar condition $\varrho_{l}(x) \leq c \delta(x)$ holds at $x \in[a, y]$. Writing $x=(1-t) a+t y$ with $0 \leq t \leq 1$ we have

$$
\delta(x)=(1-t) \delta(a)+t \delta(y) \geq t \delta(y) \geq t|a-b| / c
$$

by (b). Hence $\varrho_{l}(x) \leq|a-x|=t|a-b| \leq c \delta(c)$.

4. Let $T \subset E$ be a closed affine subspace with codimension at least 2 . Then $G=E \backslash T$ is a domain.

If $a, b \in G$, there is a half space $H$ such that $T \subset \partial H$ and $a, b \in \bar{H}$. Applying the result in Example 2 we see that $G$ is $c$-uniform for all $c>2$.

In particular, the complement of a point is $c$-uniform.

10.5. Suppose that $G \subsetneq E$ is a $\mathrm{QH}$ c-uniform domain. We shall prove in 10.9 that if $\gamma$ is a $c^{\prime}$-neargeodesic in $\mathrm{G}$, then $\gamma$ has a length cigar neighborhood $\operatorname{cig}_{l}\left(\gamma, c_{1}\right) \subset G$, where $c_{1}=c_{1}\left(c, c^{\prime}\right)$. In view of 9.4, it follows that $\mathrm{QH} c$-uniformity implies $c_{1}$-uniformity. A diameter version of this will be given in 10.15, where $\gamma$ is assumed to be a $\left(c^{\prime}, h\right)$-solid arc in $G$.

To prove these results we need three lemmas, which describe the behavior of an arc lying near the boundary of a domain. For sets $A, B \subset E$ we write $A+B=\{a+b: a \in$ $A, b \in B\}$.

10.6. Lemma. Suppose that $G \subsetneq E$ and that $r>0,0 \leq h \leq R$. Let $\gamma$ be an arc in $\partial G+\bar{B}(r)$ with $l_{k}(\gamma, h) \leq R$. Then $d(\gamma) \leq M R r$ with $M=M(h)$. If $h=0$, then $l(\gamma) \leq R r$

Proof. The case $h=0$ is easy, since then

$$
\frac{l(\gamma)}{r} \leq \int_{\gamma} \frac{|d x|}{\delta(x)}=l_{k}(\gamma) \leq R
$$

Assume that $h>0$. We show that the lemma holds with $M=2\left(e^{h}-1\right) / h$. Let $a, b \in \gamma$ be such that $d(\gamma)=|a-b|$. We consider two cases.

Case 1. $k(a, b) \leq h$. Now $3.7(2)$ yields

$$
d(\gamma)=|a-b| \leq\left(e^{k(a, b)}-1\right) \delta(a) \leq\left(e^{h}-1\right) r=h M r / 2 \leq R M r / 2 .
$$

Case 2. $k(a, b)>h$. Choose a sequence $\left(x_{0}, \ldots, x_{m+1}\right)$ of successive points of $\gamma$ such that $x_{0}=a, x_{m+1}=b, k\left(x_{j-1}, x_{j}\right)=h$ for $1 \leq j \leq m$, and $k\left(x_{m}, x_{m+1}\right) \leq h$. Then $m \geq 1$. Since $\left(x_{0}, \ldots, x_{m}\right)$ is $h$-coarse, we have

$$
m h=\sum_{j=1}^{m} k\left(x_{j-1}, x_{j}\right) \leq l_{k}(\gamma, h) \leq R .
$$

Since $3.7(2)$ gives

$$
\left|x_{j-1}-x_{j}\right| \leq \delta\left(x_{j-1}\right)\left(e^{k\left(x_{j-1}, x_{j}\right)}-1\right) \leq r\left(e^{h}-1\right)
$$

for $1 \leq j \leq m+1$, we get

$$
d(\gamma)=|a-b| \leq \sum_{j=1}^{m+1}\left|x_{j-1}-x_{j}\right| \leq(m+1) r\left(e^{h}-1\right) \leq m r M h \leq M R r
$$


10.7. Lemma. Suppose that $G$ is a $\mathrm{QH}$ c-uniform domain, that $r>0$, and that $\gamma$ is $a\left(c^{\prime}, h\right)$-solid arc in $G$ lying in $\partial G+\bar{B}(r)$. Then $d(\gamma) \leq M_{1}\left(c, c^{\prime}, h\right) r$. If $h=0$, then $l(\gamma) \leq M_{1}\left(c, c^{\prime}\right) r$

Proof. Assume first that $h>0$. Let $a_{0} \in \gamma$ be a point with maximal $\delta\left(a_{0}\right)$. Dividing $\gamma$ to two subarcs we may assume that $a_{0}$ is an endpoint, which we consider as the first point of $\gamma$. Let $b$ be the other endpoint. We first prove the special case where $\delta(b) \geq r / 2$. Write

$$
t=d(\gamma) / r, R=c c^{\prime} \log (1+2 t)
$$

If $R \leq h$, then $t \leq M_{1}$ with $M_{1}=\left(e^{h / c c^{\prime}}-1\right) / 2$. Suppose that $h \leq R$. Since $\gamma$ is $\left(c^{\prime}, h\right)$-solid and $G$ is $\mathrm{QH} c$-uniform, we get

$$
l_{k}(\gamma, h) \leq c^{\prime} k\left(a_{0}, b\right) \leq c c^{\prime} j_{G}\left(a_{0}, b\right) \leq R .
$$

By Lemma 10.6 this implies $t \leq M(h) R$. Since $t^{-1} \log (1+2 t) \rightarrow 0$ as $t \rightarrow \infty$, this gives the desired bound $t \leq M_{1}\left(c, c^{\prime}, h\right)$.

In the general case we may assume that $\delta\left(a_{0}\right)=r$. Let $N$ be the least integer with $2^{-N} r \leq \delta(b)$. For $1 \leq j \leq N$ we let $a_{j}$ denote the last point on $\gamma$ with $\delta\left(a_{j}\right) \geq 2^{-j} r$. Then $a_{N}=b$. By the special case, the $\operatorname{arcs} \gamma_{j}=\gamma\left[a_{j-1}, a_{j}\right]$ satisfy the inequality $d\left(\gamma_{j}\right) \leq$ $2^{-j+1} M_{1} r$ with $M_{1}$ as above. Hence

$$
d(\gamma) \leq \sum_{j=1}^{N} d\left(\gamma_{j}\right) \leq 2 M_{1} r
$$

The case $h=0$ is proved similarly, replacing diameter by length.

10.8. Lemma. For each $c \geq 1, c^{\prime} \geq 1$ and $h \geq 0$ there is a number $q=q\left(c, c^{\prime}, h\right) \in$ $(0,1)$ with the following property: Let $G$ be a $\mathrm{QH}$ c-uniform domain, let $\gamma \subset G$ be $a\left(c^{\prime}, h\right)$ solid arc with endpoints $a_{0}, a_{1}$ and let $x \in \gamma$ be a point with $\delta(x) \leq q \delta\left(a_{0}\right)$. Then for $\gamma_{x}=$ $\gamma\left[x, a_{1}\right]$, we have $d\left(\gamma_{x}\right) \leq M_{2}\left(c, c^{\prime}, h\right) \delta(x)$ if $h>0$, and $l\left(\gamma_{x}\right) \leq M_{2}\left(c, c^{\prime}\right) \delta(x)$ if $h=0$.

Proof. Let $M_{1}=M_{1}\left(c, c^{\prime}, h\right)$ be the constant given by 10.7 and write $K=2(h \vee$ $\left.c c^{\prime} \log \left(1+M_{1}\right)\right)$. We show that the lemma holds with $q=e^{-K}$.

In the situation of the lemma we write $r=\delta(x) / q$. Then $r \leq \delta\left(a_{0}\right)$. It suffices to show that $\gamma_{x} \subset \partial G+\bar{B}(r)$, since then 10.7 implies the lemma with $M_{2}=M_{1} / q$.

Assume that $\gamma_{x} \not \subset \partial G+\bar{B}(r)$. Since $\delta\left(a_{0}\right) \geq r$, there are $x_{1}, x_{2} \in \gamma$ such that $\delta\left(x_{1}\right)=$ $\delta\left(x_{2}\right)=r$ and $x \in \alpha \subset G \cap(\partial G+\bar{B}(r))$ where $\alpha=\gamma\left[x_{1}, x_{2}\right]$. Then $\left|x_{1}-x_{2}\right| \leq d(\alpha) \leq M_{1} r$ by 10.7. By the QH uniformity of $G$ and by the solidity of $\gamma$ we obtain

$$
l_{k}\left(\gamma\left[x_{1}, x\right], h\right) \leq l_{k}(\alpha, h) \leq c^{\prime} k\left(x_{1}, x_{2}\right) \leq c c^{\prime} j_{G}\left(x_{1}, x_{2}\right) \leq c c^{\prime} \log \left(1+M_{1}\right) .
$$

By 3.7(1) and 9.11(2) this implies

$$
\log \left(1+\frac{\left|x_{1}-x\right|}{q r}\right) \leq k\left(x_{1}, x\right) \leq k\left(\gamma\left[x_{1}, x\right]\right) \leq h \vee l_{k}\left(\gamma\left[x_{1}, x\right], h\right) \leq K / 2
$$

On the other hand, $\left|x_{1}-x\right| \geq \delta\left(x_{1}\right)-\delta(x)=(1-q) r$, and hence

$$
1+\frac{\left|x_{1}-x\right|}{q r} \geq \frac{1}{q} \text {. }
$$

These estimates give the contradiction $K=\log (1 / q) \leq K / 2$. 
10.9. Cigar Theorem. Suppose that $G$ is $\mathrm{QH}$ c-uniform and that $\gamma \subset G$ is a $c^{\prime}$ neargeodesic with endpoints $a_{0}$ and $a_{1}$. Then there is $c_{1}=c_{1}\left(c, c^{\prime}\right)$ such that

(1) $\operatorname{cig}_{l}\left(\gamma, c_{1}\right) \subset G$,

(2) $l(\gamma) \leq c_{1}\left|a_{0}-a_{1}\right|$.

Proof. (1) Let $x_{0} \in \gamma$ be a point with maximal $\delta\left(x_{0}\right)$. By symmetry, it suffices to find an estimate

$$
l\left(\gamma\left[a_{0}, x\right]\right) \leq c_{1} \delta(x)
$$

for all $x \in \gamma\left[a_{0}, x_{0}\right]$. Let $q=q\left(c, c^{\prime}, 0\right) \in(0,1)$ be the number given by 10.8. If $\delta(x) \leq$ $q \delta\left(x_{0}\right)$, then 10.8 implies (10.10) with $c_{1}=M_{2}\left(c, c^{\prime}\right)$. If $\delta(x) \geq q \delta\left(x_{0}\right)$, we apply 10.7 with $r=\delta\left(x_{0}\right)$ and obtain (10.10) with $c_{1}=M_{1}\left(c, c^{\prime}\right) / q$.

(2) We may assume that $\delta\left(a_{0}\right) \leq \delta\left(a_{1}\right)$. Let $c_{2}, c_{3}, \ldots$ denote positive constants depending only on $c$ and $c^{\prime}$. Writing $t=\left|a_{0}-a_{1}\right|$ we look for an estimate

$$
l(\gamma) \leq c_{2} t
$$

Set $r=\delta\left(a_{0}\right)$. We consider two cases.

Case 1. $r \leq t$. We may assume that $l(\gamma)>2 t$. Choose points $b_{0}, b_{1} \in \gamma$ such that

$$
l\left(\gamma\left[a_{0}, b_{0}\right]\right)=l\left(\gamma\left[a_{1}, b_{1}\right]\right)=t .
$$

By part (1) we have $t \leq c_{1} \delta\left(b_{j}\right)$ for $j=0,1$. With the notation of 3.6 we obtain

$$
r_{G}\left(b_{0}, b_{1}\right) \leq \frac{\left|b_{0}-a_{0}\right|+\left|a_{0}-a_{1}\right|+\left|a_{1}-b_{1}\right|}{\delta\left(b_{0}\right) \wedge \delta\left(b_{1}\right)} \leq \frac{3 t}{t / c_{1}}=3 c_{1} .
$$

Since $G$ is $\mathrm{QH} c$-uniform, this implies

$$
k\left(b_{0}, b_{1}\right) \leq c j_{G}\left(b_{0}, b_{1}\right) \leq c \log \left(1+3 c_{1}\right) .
$$

For each $x \in \gamma\left[b_{0}, b_{1}\right]$ we get

$$
k\left(x, b_{0}\right) \leq l_{k}\left(\gamma\left[b_{0}, b_{1}\right]\right) \leq c^{\prime} k\left(b_{0}, b_{1}\right) \leq c c^{\prime} \log \left(1+3 c_{1}\right)=c_{3} .
$$

By 3.7(2) this implies that $\left|x-b_{0}\right| \leq \delta\left(b_{0}\right)\left(e^{c_{3}}-1\right)$. Since

$$
\delta\left(b_{0}\right) \leq \delta\left(a_{0}\right)+\left|a_{0}-b_{0}\right| \leq r+t \leq 2 t,
$$

this yields

$$
\delta(x) \leq \delta\left(b_{0}\right)+\left|x-b_{0}\right| \leq \delta\left(b_{0}\right) e^{c_{3}} \leq 2 t e^{c_{3}}=c_{4} t .
$$

Integration along $\gamma\left[b_{0}, b_{1}\right]$ gives

$$
c^{\prime} k\left(b_{0}, b_{1}\right) \geq l_{k}\left(\gamma\left[b_{0}, b_{1}\right]\right) \geq l\left(\gamma\left[b_{0}, b_{1}\right]\right) / c_{4} t .
$$

By (10.12), this implies that $l\left(\gamma\left[b_{0}, b_{1}\right]\right) \leq c_{5} t$, and (10.11) follows with $c_{2}=c_{5}+2$.

Case 2. $r \geq t$. We show that (10.11) is true with $c_{2}=\max \left\{u\left(e^{2 c^{\prime} / u}-1\right): u \geq 1\right\}$. This number is independent of $c$, and, in fact, the QH uniformity of $G$ is not needed in this case.

Since $\delta\left(a_{1}\right) \geq \delta\left(a_{0}\right)=r$, we have $\delta(x) \geq r / 2$ for all $x \in\left[a_{0}, a_{1}\right]$. Integration along $\left[a_{0}, a_{1}\right]$ gives

$$
k\left(a_{0}, a_{1}\right) \leq 2 t / r
$$


Set $\lambda=l(\gamma)$ and let $\gamma^{0}:[0, \lambda] \rightarrow \gamma$ be the arclength parametrization of $\gamma$ with $\gamma^{0}(0)=a_{0}$. Since $\delta(\alpha(s)) \leq \delta\left(a_{0}\right)+\left|a_{0}-\alpha(s)\right| \leq r+s$ for all $0 \leq s \leq \lambda$, we get

$$
l_{k}(\gamma) \geq \int_{0}^{\lambda} \frac{d s}{r+s}=\log (1+\lambda / r) .
$$

Since $\gamma$ is a $c^{\prime}$-neargeodesic, this and (10.13) give

$$
\log (1+\lambda / r) \leq c^{\prime} k\left(a_{0}, a_{1}\right) \leq 2 c^{\prime} t / r .
$$

Setting $u=r / t \geq 1$ we thus have $1+\lambda / t u \leq e^{2 c^{\prime} / u}$, and (10.11) follows.

10.14. TheOREM. If $\gamma$ is a $c^{\prime}$-neargeodesic in a $\mathrm{QH}$ c-uniform domain, then $\gamma$ is $c_{1}$-quasiconvex in the norm metric with $c_{1}=c_{1}\left(c, c^{\prime}\right)$.

10.15. Coarse Cigar Theorem. Suppose that $G$ is a $\mathrm{QH}$ c-uniform domain and that $\gamma$ is a $\left(c^{\prime}, h\right)$-solid arc in $G$ with endpoints $a_{0}$ and $a_{1}$. Then there is $c_{1}=c_{1}\left(c, c^{\prime}, h\right)$ such that

(1) $\operatorname{cig}_{d}\left(\gamma, c_{1}\right) \subset G$,

(2) $d(\gamma) \leq c_{1}\left(\left|a_{1}-a_{0}\right| \vee 2 r\left(e^{h}-1\right)\right)$ where $r=\delta\left(a_{0}\right) \wedge \delta\left(a_{1}\right)$.

Proof. We prove only (1), since (2) is not needed in this article. Its proof is given in [II, 6.22].

Choose a point $x_{0} \in \gamma$ with maximal $\delta\left(x_{0}\right)$. Let $x \in \gamma\left[a_{0}, x_{0}\right]$. It suffices to find an estimate

$$
d\left(\gamma\left[a_{0}, x\right]\right) \leq c_{1} \delta(x)
$$

$c_{1}=c_{1}\left(c, c^{\prime}, h\right)$. Let $q=q\left(c, c^{\prime}, h\right) \in(0,1)$ be the number given by 10.8. If $\delta(x) \leq q \delta\left(x_{0}\right)$, then 10.8 implies (10.16) with $c_{1}=M_{2}\left(c, c^{\prime}, h\right)$. If $\delta(x) \geq q \delta\left(x_{0}\right)$, we can apply 10.7 with $r \mapsto \delta\left(x_{0}\right), \gamma \mapsto \gamma\left[a_{0}, x\right]$ and get (10.16) with $c_{1}=M_{1}\left(c, c^{\prime}, h\right) / q$.

10.17. TheOREM. For a domain $G \subsetneq E$, the following conditions are quantitatively equivalent:

(1) $G$ is c-uniform,

(2) $G$ is $\mathrm{QH}$ c-uniform,

(3) $k_{G} \leq c j_{G}+d$, where $c$ and $d$ are constants.

Proof. We show that $(3) \Rightarrow(2) \Rightarrow(1) \Rightarrow(3)$. The implication $(2) \Rightarrow(1)$ follows from 9.4 and 10.9. Assume that (3) holds, let $a, b \in G$, and set $r=r_{G}(a, b)$. If $r \leq 1 / 2$, then $r \log 2 \leq \log (1+r)$, and 3.9 implies that $k(a, b) \leq 2 r \leq(2 / \log 2) j_{G}(a, b)$. If $r \geq 1 / 2$, then $j_{G}(a, b) \geq \log (3 / 2)$. Hence

$$
\frac{k(a, b)}{j_{G}(a, b)} \leq c+\frac{d}{\log (3 / 2)},
$$

and we obtain (2). It remains to prove that (1) $\Rightarrow(3)$.

Assume that (1) is true, and let $a, b \in G$. We want to find an estimate

$$
k(a, b) \leq c_{1} j_{G}(a, b)+d,
$$


where $c_{1}$ and $d$ depend only on $c$. Let $\gamma$ be an arc joining $a$ and $b$ and satisfying the uniformity conditions of 10.2. Let $x_{0} \in \gamma$ be the point bisecting the length $\lambda$ of $\gamma$. We may assume that $\delta(a) \leq 2|a-b|$, since otherwise 3.9 gives $k(a, b) \leq 1$, and (10.18) holds with $c=0, d=1$. Setting $\delta=\delta(a) \wedge \delta(b)$ we have $\delta / 2 \leq|a-b| \leq \lambda$. Choose points $a_{1}, b_{1} \in \gamma$ with $l\left(\gamma\left[a, a_{1}\right]\right)=l\left(\gamma\left[b, b_{1}\right]\right)=\delta / 4$. Since $\left|a_{1}-a\right| \leq \delta(a) / 4$, Theorem 3.9 yields $k\left(a_{1}, a\right) \leq 1 / 2$, and similarly $k\left(b_{1}, b\right) \leq 1 / 2$. Setting $\beta=\gamma\left[a_{1}, x_{0}\right]$ we obtain by the cigar condition $10.2(1)$

$$
k\left(a_{1}, x_{0}\right) \leq l_{k}(\beta) \leq c \int_{\beta} \frac{|d x|}{l(\gamma[a, x])}=c \int_{\delta / 4}^{\lambda / 2} \frac{d s}{s}=c \log \frac{2 \lambda}{\delta} .
$$

Since a similar estimate holds for $k\left(b_{1}, x_{0}\right)$, we get by the turning condition $10.2(2)$

$$
\begin{aligned}
k(a, b) & \leq k\left(a, a_{1}\right)+k\left(a_{1}, x_{0}\right)+k\left(x_{0}, b_{1}\right)+k\left(b_{1}, b\right) \\
& \leq 1+2 c \log \frac{2 \lambda}{\delta} \leq 1+2 c \log \frac{2 c|a-b|}{\delta} .
\end{aligned}
$$

Since $\log (M t) \leq M \log (1+t)$ for all $M \geq 1, t \geq 0$, this implies (10.18) with $c_{1}=4 c^{2}$, $d=1$.

10.19. Invariance of uniform domains. Suppose that $G$ is a $c$-uniform domain and that $f: G \rightarrow G^{\prime}$ is a homeomorphism. It is easy to see that if $f$ is $M$-bilipschitz, then $G^{\prime}$ is $c^{\prime}$-uniform with $c^{\prime}=M^{2} c$. It is less obvious that the property of being $c$-uniform is quantitatively invariant under $\eta$-quasisymmetric and even under $\eta$-quasimöbius maps. The proof of this is based on the following result:

10.20. Lemma. Suppose that $G \neq E, G^{\prime} \neq E^{\prime}$, and that $f: G \rightarrow G^{\prime}$ is an $\eta$ quasimöbius homeomorphism. Then

$$
j_{G^{\prime}}(f a, f b) \leq M j_{G}(a, b)+C
$$

for all $a, b \in G$, where $M \geq 0$ and $C \geq 0$ depend only on $\eta$.

Proof. We first consider two special cases.

Case 1. $0 \notin G$ and $f$ is the restriction of the inversion $u(x)=x /|x|^{2}$. Let $a, b \in G$ and set $r=r_{G}(a, b), r^{\prime}=r_{G^{\prime}}(u a, u b)$; see 3.6 for the notation. We may assume that $\delta^{\prime}(u a) \leq \delta^{\prime}(u b)$. Choose $x \in \partial G$ such that $|u x-u a| \leq 2 \delta^{\prime}(u a)$. By (5.9) we obtain

Since

$$
r^{\prime}=\frac{|u a-u b|}{\delta^{\prime}(u a)} \leq 2 \frac{|u a-u b|}{|u a-u x|} \leq \frac{18|a-b||x|}{|a-x||b|} .
$$

$$
|x| \leq|x-a|+|a-b|+|b|,|x-a| \geq \delta(a),|b| \geq \delta(b)
$$

this implies

$$
r^{\prime} \leq 18|a-b|\left(\frac{1}{\delta(b)}+\frac{|a-b|}{\delta(a) \delta(b)}+\frac{1}{\delta(a)}\right) \leq 18 r(2+r) .
$$

Hence $1+r^{\prime} \leq 18(1+r)^{2}$, and we obtain (10.21) with the universal constants $M=2, C=$ $\log 18$.

Case 2. $f$ is $\eta$-quasisymmetric. By 6.14 , we may assume that $\eta(t)=C_{1}\left(t^{\alpha} \vee t^{1 / \alpha}\right)$, where $C_{1} \geq 1,0<\alpha \leq 1$. Let $a, b \in G$. We may again assume that $\delta^{\prime}(f a) \leq \delta^{\prime}(f b)$. By 
6.12, $f$ extends to an $\eta$-quasisymmetric homeomorphism $\bar{f}: \bar{G} \rightarrow \overline{G^{\prime}}$. Choose $x \in \partial G$ such that $|f a-\bar{f} x| \leq 2 \delta^{\prime}(f a)$. Setting $r=r_{G}(a, b)$ we have $|a-b| \leq r|a-x|$, and hence

$$
r_{G^{\prime}}(f a, f b)=\frac{|f a-f b|}{\delta^{\prime}(f a)} \leq 2 \frac{|f a-f b|}{|f a-\bar{f} x|} \leq 2 \eta\left(\frac{|a-b|}{|a-x|}\right) \leq 2 C_{1}\left(r^{\alpha} \vee r^{1 / \alpha}\right) .
$$

If $r \leq 1$, then (10.21) holds with $M=0, C=\log \left(1+2 C_{1}\right)$. If $r \geq 1$, then

$$
1+r_{G^{\prime}}(f a, f b) \leq 1+2 C_{1} r^{1 / \alpha} \leq 2 C_{1}(1+r)^{1 / \alpha},
$$

and we obtain (10.21) with $M=1 / \alpha, C=\log 2 C_{1}$.

In the general case we first apply 6.24 to extend $f$ to a homeomorphism $\bar{f}: \bar{G} \rightarrow \overline{G^{\prime}}$. By auxiliary translations we may assume that $0 \in \partial G$ and that $\bar{f}(0)$ is either 0 or $\infty$. Then we can use auxiliary inversions and Case 1 to normalize the situation so that $\infty \in \partial G$ and $\bar{f}(\infty)=\infty$. Then $f$ is $\eta$-QS, and the result follows from Case 2 .

10.22. TheOREM. Suppose that $f: G \rightarrow G^{\prime}$ is an $\eta$-quasimöbius homeomorphism and that $G$ is a c-uniform domain. Then $G^{\prime}$ is $c_{1}$-uniform with $c_{1}=c_{1}(c, \eta)$.

Proof. By 7.17 and 4.7, $f$ is $\varphi$-FQC and $c$-coarsely $M$-QH with $\varphi, M, C$ depending only on $\eta$. If $G=E$, then $G^{\prime}=E^{\prime}$ by 7.14. Assume that $G \neq E$ and hence $G^{\prime} \neq E^{\prime}$. Let $a, b \in G$. Applying 10.20 to $f^{-1}: G^{\prime} \rightarrow G$ we can write

$$
j_{G}(a, b) \leq M_{1} j_{G^{\prime}}(f a, f b)+C_{1}
$$

with $M_{1}, C_{1}$ depending only on $\eta$. Since $G$ is $\mathrm{QH} c_{0}$-uniform with $c_{0}=c_{0}(c)$ by 10.17 , we obtain

$$
k^{\prime}(f a, f b) \leq M k(a, b)+C \leq M c_{0} j_{G}(a, b)+C \leq M M_{1} c_{0} j_{G^{\prime}}(f a, f b)+M C_{1} c_{0}+C,
$$

and the theorem follows from 10.17.

10.23. Problem. Does 10.22 remain true if the quasimöbius condition is replaced by quasimöbius rel $\partial G$ ?

10.24. Notes. Uniform domains in $R^{n}$ were introduced by O. Martio and J. Sarvas [MS] in 1979 and independently by P. Jones [ $\left.\mathrm{Jo}_{1}\right]$, and they have turned out to be very useful in various questions in analysis. Another useful concept is that of a John domain, which is obtained by removing the turning condition 10.2(2) from the definition of a uniform domain. The equivalence of uniform and QH uniform domains in $R^{n}$ was proved by F. W. Gehring and B. Osgood [GO]. Several ideas of 10.4 are due to P. Alestalo [Al].

\section{Maps of uniform domains}

11.1. Summary. In this section we study homeomorphisms $f: G \rightarrow G^{\prime}$ where $G$ and $G^{\prime}$ are uniform domains. We show that if $f$ is coarsely quasihyperbolic, then $f$ extends to a homeomorphism $\bar{f}: \bar{G} \rightarrow \overline{G^{\prime}}$, and $\bar{f}$ is quasimöbius rel $\partial G$. In particular, $\bar{f} \mid \partial G$ is quasimöbius. If $f$ is freely quasiconformal, then $\bar{f}$ is quasimöbius. All these results are quantitative.

11.2. Cluster sets. Suppose that $f: G \rightarrow E^{\prime}$ is a continuous map and that $x_{0} \in \partial G$. Remember that $\partial G$ is taken in $\dot{E}$, and the case $x_{0}=\infty$ is possible. The cluster set of $f$ 
at $x_{0}$ is

$$
\operatorname{clus}\left(x_{0}, f\right)=\cap\left\{\operatorname{cl} f[U \cap G]: x_{0} \in U, U \text { open in } \dot{E}\right\} .
$$

Equivalently, a point $y \in \dot{E}^{\prime}$ is in clus $\left(x_{0}, f\right)$ if and only if there is a sequence $\left(x_{j}\right)$ in $G$ such that $x_{j} \rightarrow x_{0}$ and $f x_{j} \rightarrow y$.

The set clus $\left(x_{0}, f\right)$ is a singleton $\left\{y_{0}\right\}$ if and only if $f$ has the limit $y_{0}$ at $x_{0}$. If $f$ is a homeomorphism onto a domain $G^{\prime}$, then clus $\left(x_{0}, f\right) \subset \partial G^{\prime}$.

If $\operatorname{dim} E^{\prime}<\infty$, the cluster set is always nonempty. If $\operatorname{dim} E^{\prime}=\infty$, the cluster set can be empty even if $f$ is an FQC map. For example, the map $h: H \rightarrow T^{\prime}$ of a half space onto a broken tube considered in 8.15 has empty cluster sets at 0 and at $\infty$.

The crux of the theory of this section is the following result, the proof of which makes effective use of neargeodesics, solid arcs, and both cigar theorems of Section 10.

11.3. Fundamental Lemma. Suppose that

(1) $G \neq E$ and $G^{\prime} \neq E^{\prime}$ are unbounded c-uniform domains,

(2) $f: G \rightarrow G^{\prime}$ is $C$-coarsely $M$-QH,

(3) $\infty \in \operatorname{clus}(\infty, f)$,

(4) $x, a, b$ are distinct points in $G,|a-x| \leq|b-x|$, and $k(b, x) \geq(2 C) \vee \frac{1}{2}$.

Then $|f a-f x| \leq H|f b-f x|$ where $H=H(M, C, c)$.

Proof. Performing auxiliary similarities we can normalize the situation so that $x=$ $0, f x=0,|b|=1,|f b|=1$. Now $|a| \leq|b|=1$ and we look for an estimate

$$
|f a| \leq H(M, C, c) \text {. }
$$

We first show that

$$
\delta^{\prime}(0) \leq 8 M
$$

If $\delta^{\prime}(0) \leq 2,(11.5)$ is trivially true. Assume that $\delta^{\prime}(0) \geq 2$. Since $|f b|=1$, Theorem 3.9 gives $k^{\prime}(f b, 0) \leq 2 / \delta^{\prime}(0)$. Since

$$
\frac{1}{4}+C \leq(2 C) \vee \frac{1}{2} \leq k(b, 0) \leq M k^{\prime}(f b, 0)+C,
$$

this yields (11.5).

We let $c_{1}, c_{2}, \ldots$ denote constants $c_{j} \geq 1$ depending only on $(M, C, c)$. By 10.17 and by the cigar theorem 10.9 , there is $c_{1}$ such that each 2-neargeodesic in $G$ or in $G^{\prime}$ satisfies the uniformity conditions of 10.2 with the constant $c_{1}$ in $G$ or in $G^{\prime}$, respectively. Since $\infty \in \operatorname{clus}(\infty, f)$, there is $y \in G$ such that

$$
|y| \geq 2,|f y| \geq|f a| .
$$

Applying 9.4 we join the points $f a$ and $f y$ by a 2 -neargeodesic $\alpha^{\prime}$ in $G^{\prime}$. Let $z \in \alpha^{\prime}$ be a point with $|z|=d\left(0, \alpha^{\prime}\right)$. Since

$$
|z-f a| \geq|f a|-|z|,|z-f y| \geq|f y|-|z| \geq|f a|-|z|,
$$

the cigar condition and (11.5) give

$$
|f a|-|z| \leq c_{1} \delta^{\prime}(z) \leq c_{1}\left(\delta^{\prime}(0)+|z|\right) \leq c_{1}(8 M+|z|) .
$$


We may assume that $|f a| \geq 16 c_{1} M$, since otherwise (11.4) is clear. Then $|f a|-8 c_{1} M \geq$ $|f a| / 2$, and (11.6) implies

$$
|f a| \leq 2\left(1+c_{1}\right)|z| \leq 4 c_{1} d\left(0, \alpha^{\prime}\right) .
$$

We join 0 and $f b$ by a 2-neargeodesic $\beta^{\prime}$ in $G^{\prime}$. Write $\alpha=f^{-1} \alpha^{\prime}, \beta=f^{-1} \beta^{\prime}$, and choose points $a_{0} \in \alpha$ and $b_{0} \in \beta$ with $\left|a_{0}\right|=3 / 2,\left|b_{0}\right|=1 / 2$. By the solid arc theorem 9.18 , the $\operatorname{arcs} \alpha$ and $\beta$ are $\left(c_{2}, h\right)$-solid with $h=h(M, C)$. By the coarse cigar theorem 10.15 , there is $c_{3}$ such that $\operatorname{cig}_{d}\left(\alpha, c_{3}\right)$ and $\operatorname{cig}_{d}\left(\beta, c_{3}\right)$ lie in $G$. Hence

$$
\delta\left(a_{0}\right) \wedge \delta\left(b_{0}\right) \geq 1 / 2 c_{3} .
$$

Since $G$ is QH $c_{4}$-uniform by 10.17 and since $\left|a_{0}-b_{0}\right| \leq 2$, this implies

$$
k\left(a_{0}, b_{0}\right) \leq c_{4} j_{G}\left(a_{0}, b_{0}\right) \leq c_{4} \log \left(1+4 c_{3}\right)=c_{5} .
$$

Thus $k^{\prime}\left(f a_{0}, f b_{0}\right) \leq M c_{5}+C=c_{6}$. The turning condition $l\left(\beta^{\prime}\right) \leq c_{1}|f b-0|=c_{1}$ implies that $\left|f b_{0}\right| \leq c_{1}$. Applying (11.5) and 3.7(2) we get

$$
\begin{aligned}
& \delta^{\prime}\left(f b_{0}\right) \leq \delta^{\prime}(0)+\left|f b_{0}\right| \leq 8 M+c_{1}, \\
& \left|f a_{0}-f b_{0}\right| \leq \delta^{\prime}\left(f b_{0}\right) e^{k^{\prime}\left(f a_{0}, f b_{0}\right)} \leq\left(8 M+c_{1}\right) e^{c_{6}}=c_{7} .
\end{aligned}
$$

By (11.7) this yields

$$
|f a| \leq 4 c_{1}\left|f a_{0}\right| \leq 4 c_{1}\left(\left|f a_{0}-f b_{0}\right|+\left|f b_{0}\right|\right) \leq 4 c_{1}\left(c_{7}+c_{1}\right),
$$

and we obtain (11.4).

11.8. Theorem. Suppose that $G \neq E$ and $G^{\prime} \neq E^{\prime}$ are c-uniform domains and that $f: G \rightarrow G^{\prime}$ is $C$-coarsely $M$-QH. Then $f$ extends to a homeomorphism $\bar{f}: \bar{G} \rightarrow \overline{G^{\prime}}$, and $\bar{f}$ is $\eta$-quasimöbius rel $\partial G$ with $\eta$ depending only on $(M, C, c)$. In particular, $f \mid \partial G$ is $\eta$-quasimöbius.

PROOF. In the first part of the theorem, it suffices to show that $f$ has a limit at each $x_{0} \in \partial G$. We may assume that $x_{0} \neq \infty$ by an auxiliary inversion and by 10.22 . Moreover, we may assume that $0 \in G, f(0)=0, \delta(0)=1$ and $\delta^{\prime}(0)=1$. We assume that $f$ has no limit (finite or infinite) at $x_{0}$ and look for a contradiction.

Set $D(r)=f\left[B\left(x_{0}, r\right) \cap G\right]$. There is $q>0$ such that $d(D(r))>q$ for all $r>0$, since otherwise the limit of $f$ at $x_{0}$ exists by the completeness of $E^{\prime}$. Since $\infty$ is not the limit of $f$ at $x_{0}$, there is $R \geq q$ such that $D(r)$ meets $B(R)$ for all $r>0$. We let $c_{1}, c_{2}, \ldots$ denote constants $c_{j} \geq 1$ depending only on $M, C, c, q, R$.

Let $r>0$ and choose points $x, y \in G \cap B\left(x_{0}, r\right)$ such that $|f x|<R$ and $|f x-f y| \geq q / 2$. Applying 9.4 we join $x$ and $y$ by a 2-neargeodesic $\gamma$ in $G$. Choose $z \in \gamma$ with $|f z-f x|=$ $q / 4$. Then $|f z-f y| \geq q / 4$. The $\operatorname{arc} f \gamma$ is $\left(c_{1}, h\right)$-solid in $G^{\prime}$ with $h=h(M, C, c)$ by 9.18 . From the coarse cigar theorem 10.15 it follows that $\operatorname{cig}_{d}\left(f \gamma, c_{2}\right) \subset G^{\prime}$ for some $c_{2}$. Hence

$$
\delta^{\prime}(f z) \geq q / 4 c_{2} .
$$

We join 0 and $f z$ by a 2-neargeodesic $\beta^{\prime}$ in $G^{\prime}$. By the cigar theorem 10.9 , there is $c_{3}$ such that

$$
\operatorname{cig}_{l}\left(\beta^{\prime}, c_{3}\right) \subset G^{\prime}, l\left(\beta^{\prime}\right) \leq c_{3}|f z| .
$$


Since $\delta^{\prime}(0)=1$, the first condition and (11.9) give a lower bound $\delta^{\prime}(w) \geq 1 / c_{4}$ for all $w \in \beta^{\prime}$. Hence

$$
k^{\prime}(0, f z) \leq l_{k}\left(\beta^{\prime}\right) \leq c_{4} l\left(\beta^{\prime}\right) \leq c_{4} c_{3}|f z|
$$

Since

$$
|f z| \leq|f z-f x|+|f x| \leq q / 4+R<2 R,
$$

this yields $k^{\prime}(0, f z) \leq 2 R c_{4} c_{3}=c_{5}$, and hence

$$
k(0, z) \leq M c_{5}+C=c_{6} .
$$

On the other hand, the cigar theorem also gives $l(\gamma) \leq c_{3}|x-y| \leq 2 c_{3} r$, and hence

$$
\delta(z) \leq\left|z-x_{0}\right| \leq|z-x|+\left|x-x_{0}\right| \leq l(\gamma)+r \leq 2 c_{3} r+r=c_{7} r .
$$

These estimates and 3.7(1) imply that

$$
\log \frac{1}{c_{7} r} \leq \log \frac{\delta(0)}{\delta(z)} \leq k(0, z) \leq c_{6} .
$$

As $r \rightarrow 0$, this gives the desired contradiction.

To prove the second part of the theorem, let $Q=(a, b, c, d)$ be a quadruple in $\bar{G}$ with $a, d \in \partial G$. By symmetry and by 6.28 , it suffices to find an estimate $\tau(f Q) \leq \eta(\tau(Q))$ with some increasing $\eta:[0, \infty) \rightarrow[0, \infty)$ depending only on $v=(M, C, c)$. Performing auxiliary inversions we may assume that $d=\infty$ and $\bar{f} d=\infty$.

Choose sequences $\left(a_{n}\right),\left(b_{n}\right),\left(c_{n}\right)$ in $G$ converging to $a, b, c$, respectively, such that the points $a_{n}, b_{n}, c_{n}$ are distinct for each $n$. Set $T_{n}=\left(a_{n}, b_{n}, c_{n}\right)$. Since we have $\varrho\left(T_{n}\right)=$ $\left|a_{n}-b_{n}\right| /\left|a_{n}-c_{n}\right| \rightarrow \tau(Q)$ and $\varrho\left(f T_{n}\right) \rightarrow \tau(f Q)$, it suffices to find an estimate

$$
\varrho\left(f T_{n}\right) \leq \eta\left(\varrho\left(T_{n}\right)\right)
$$

with $\eta=\eta_{v}$. Setting $\lambda=2 C \vee \frac{1}{2}$ and observing that $\delta\left(a_{n}\right) \rightarrow 0$ we may assume that

$$
\left|a_{n}-c_{n}\right|>e^{\lambda} \delta\left(a_{n}\right) \text { for all } n \text {. }
$$

Fix $n \in \mathbf{N}$. Applying the $c$-uniformity of $G$ we join $a_{n}$ and $b_{n}$ by an arc $\gamma \subset G$ with $l(\gamma) \leq c\left|a_{n}-b_{n}\right|$. Orient $\gamma$ so that $a_{n}$ is the first point. Set $y_{0}=a_{n}$ and let $y_{1}$ be the last point of $\gamma$ with $\left|y_{1}-y_{0}\right| \leq\left|c_{n}-y_{0}\right|$. Proceeding inductively, we let $y_{j+1}$ be the last point of $\gamma$ with $\left|y_{j+1}-y_{j}\right| \leq\left|y_{j}-y_{0}\right|$, and we stop as soon as we find $y_{s}$ with $y_{s}=b_{n}$. The process is finite, since $\gamma$ is compact and since $\left|y_{j}-y_{j-1}\right| \geq\left|c_{n}-y_{0}\right|$ for all $1 \leq j \leq s-1$. Assume that $s \geq 2$. For $1 \leq j \leq s-1$ we have $\left|y_{j}-a_{n}\right| \geq\left|y_{1}-a_{n}\right|=\left|c_{n}-a_{n}\right|$. By (11.11) and by $3.7(1)$, this implies that $k\left(y_{j}, a_{n}\right) \geq \lambda$ and $k\left(c_{n}, a_{n}\right) \geq \lambda$. By the fundamental lemma 11.3 , there is $H=H(v) \geq 1$ such that

$$
\left|f y_{1}-f a_{n}\right| \leq H\left|f c_{n}-f a_{n}\right|,\left|f y_{j+1}-f y_{j}\right| \leq H\left|f y_{j}-f a_{n}\right|
$$

for $1 \leq j \leq s-1$. Hence

$$
\left|f y_{j+1}-f a_{n}\right| \leq(H+1)\left|f y_{j}-f a_{n}\right| \leq(H+1)^{j}\left|f y_{1}-f a_{n}\right| \leq H(H+1)^{j}\left|f c_{n}-f a_{n}\right|
$$

for $1 \leq j \leq s-1$. For $j=s-1$ this gives

$$
\left|f b_{n}-f a_{n}\right| \leq H(H+1)^{s-1}\left|f c_{n}-f a_{n}\right| .
$$


Clearly this is also true if $s=1$. Since

$$
c\left|a_{n}-b_{n}\right| \geq l(\gamma) \geq \sum_{j=1}^{s}\left|y_{j}-y_{j-1}\right| \geq(s-1)\left|c_{n}-a_{n}\right|,
$$

we have $s-1 \leq c \varrho\left(T_{n}\right)$. Hence $(11.10)$ is true with $\eta(t) \leq H(H+1)^{c t}$.

11.12. THEOREM. Suppose that $G$ and $G^{\prime}$ are c-uniform domains and that $f: G \rightarrow G^{\prime}$ is $\varphi$-FQC. Then $f$ is $\eta$-quasimöbius with $\eta=\eta_{\varphi, c}$.

Proof. If $G=E$ or $G^{\prime}=E^{\prime}$, the result follows from 7.14. Suppose that $G \neq$ $E, G^{\prime} \neq E^{\prime}$. Since $f$ is coarsely quasihyperbolic by 4.7 , it has a homeomorphic extension $\bar{f}: \bar{G} \rightarrow \overline{G^{\prime}}$ by 11.8. By auxiliary inversions we normalize the situation so that $\infty \in \partial G$ and $\bar{f}(\infty)=\infty$. We show that $f$ is $\eta$-quasisymmetric with $\eta=\eta_{\varphi, c}$. The domains $G$ and $G^{\prime}$ are $c$-quasiconvex. By 6.6 , it suffices to show that $f$ is weakly $H$-quasisymmetric with $H=H(\varphi, c)$.

Let $x, a, b$ be points in $G$ with $|a-x| \leq|b-x|$. By $4.7(1), f$ is $\frac{1}{4}$-coarsely $M$-QH with $M=M(\varphi)$. By the fundamental lemma 11.3, the desired inequality

$$
|f a-f x| \leq H|f b-f x|
$$

holds with $H=H(\varphi, c)$ provided that $k(b, x) \geq 1 / 2$. Suppose that $k(b, x) \leq 1 / 2$. Then $3.7(2)$ gives $|b-x| \leq q \delta(x)$ with $q=e^{1 / 2}-1<1$. Hence $a, b \in \bar{B}(x, q \delta(x))$. Since $f$ is $q$-locally $\eta$-quasisymmetric with $\eta=\eta_{\varphi}$ by 7.9 , (11.13) holds with $H=\eta(1)$.

11.14. REMARK. Alternatively, Theorem 11.12 follows from 11.8 as follows. Let $Q=$ $\left(x_{1}, x_{2}, x_{3}, x_{4}\right)$ be a quadruple in $G$. One can show that $D=G \backslash\left\{x_{1}, x_{4}\right\}$ is a $c_{0}$-uniform domain with $c_{0}=c_{0}(c)$; see [II, 6.7]. From 11.8 and from 4.7 it follows that $f$ extends to a homeomorphism $\bar{f}: \bar{G} \rightarrow \overline{G^{\prime}}$ and that $\bar{f}$ is $\eta$-quasimöbius rel $\partial D$ with $\eta=\eta_{\varphi, c}$. Hence $\tau(f Q) \leq \eta(\tau(Q))$.

11.15. TheOrem. Let $G$ be c-uniform and let $f: G \rightarrow G^{\prime}$ be $\varphi$-FQC. Then the following conditions are quantitatively equivalent:

(1) $G^{\prime}$ is $c^{\prime}$-uniform,

(2) $f$ is $\eta$-quasimöbius.

Proof. This follows from 10.22 and 11.12 .

11.16. Problem. Suppose that $G$ is $c$-uniform and that $f: \bar{G} \rightarrow \overline{G^{\prime}}$ is a homeomorphism such that $f \partial G=\partial G^{\prime}$ and $f$ is $\eta$-quasimöbius rel $\partial G$. Is $G^{\prime} c^{\prime}$-uniform with $c^{\prime}=c^{\prime}(c, \eta) ?$

11.17. REMARK. Combining 11.8 and 11.12 with 6.31 and 6.33 we get various conditions for the quasisymmetry and relative quasisymmetry of homeomorphisms between bounded domains. For example, let $B(1)$ and $B^{\prime}(1)$ be the unit balls of $E$ and $E^{\prime}$, respectively, and let $f: B(1) \rightarrow B^{\prime}(1)$ be $\varphi$-FQC with $f(0)=0$. Since balls are 2-uniform by $10.4 .1, f$ is $\theta$-quasimöbius with $\theta=\theta_{\varphi}$ by 11.12 . By $6.33, f$ is $\eta$-quasisymmetric with $\eta=\eta_{\varphi}$. Moreover, $f$ extends to a homeomorphism $\bar{f}: \bar{B}(1) \rightarrow \overline{B^{\prime}}(1)$. Let $u$ be the inversion $u x=x /|x|^{2}$. We extend $f$ by reflection to a homeomorphism $F: \dot{E} \rightarrow \dot{E}^{\prime}$ with $F(x)=u f u(x)$ for $|x| \geq 1$. In $A=\dot{E} \backslash B(1)$, the map $F$ is $\theta_{1}$-quasimöbius with 
$\theta_{1}(t)=81 \theta(81 t)$ by 6.22 . Since $F(\infty)=\infty, F \mid A$ is $\theta_{1}$-quasisymmetric. These properties imply that $F$ is $\eta_{1}$-quasisymmetric with $\eta_{1}$ depending only on $\varphi$. The proof is elementary but somewhat tedious; see [II, 7.39].

11.18. Notes. This section is from [II]. In $R^{n}$, the quasiconformal case 11.12 was known earlier, and the essential step was proved by F. W. Gehring and O. Martio [GM, $2.18,3.1]$ in 1985 . However, this proof depended heavily on finite-dimensional tools, for example, on the extension of Sobolev functions from a uniform domain to $R^{n}$, due to P. W. Jones $\left[\mathrm{JO}_{2}\right]$.

Special finite-dimensional cases of the relative theorem 11.8 were also known earlier. As mentioned in 4.11, V. A. Efremovich and E. S. Tihomirova [ET] proved in 1964 that a solid homeomorphism $F: H \rightarrow H$ of the upper half space of $R^{n+1}$ extends to a homeomorphism $\bar{F}: \bar{H} \rightarrow \bar{H}$. D. A. De-Spiller [DS] proved that the induced boundary map $f: \dot{R}^{n} \rightarrow \dot{R}^{n}$ is quasiconformal. He also proved that conversely, each quasiconformal map $f: \dot{R}^{n} \rightarrow \dot{R}^{n}$ extends to a homeomorphism $\bar{F}: \bar{H} \rightarrow \bar{H}$ whose restriction $F: H \rightarrow H$ is solid. A free version of this appears in [II, 7.26]. In $\left[\mathrm{TV}_{3}\right] \mathrm{P}$. Tukia and the author proved that $F$ can be chosen to be quasiconformal. It is not known whether the free version of this is true.

\section{Further results}

12.1. Summary. We give miscellaneous results on the free quasiworld. We first show that a $\varphi$-FQC map satisfies a Hölder condition. This result is applied to prove that the function $\varphi$ can always be chosen to be of the form $\varphi(t)=C\left(t^{\alpha} \vee t\right)$. We also consider, without proofs, removable sets for FQC and other maps.

12.2. TheOrem. Suppose that $f: G \rightarrow G^{\prime}$ is $\varphi$-FQC with $G \neq E, G^{\prime} \neq E^{\prime}$. Then $f$ satisfies the Hölder condition

$$
\frac{|f a-f b|}{\delta^{\prime}(f a)} \leq C\left(\frac{|a-b|}{\delta(a)}\right)^{\alpha} \quad \text { for all } a, b \in G \text { with }|a-b| \leq \delta(a) / 2 .
$$

The numbers $C \geq 1$ and $0<\alpha \leq 1$ depend only on $\varphi$.

Proof. By part $(1) \Rightarrow(3)$ of $7.9, f$ is $\frac{1}{2}$-locally $\eta$-quasisymmetric with $\eta=\eta_{\varphi}$. Fix $a \in G$ and define a homeomorphism $g: \bar{B}(1 / 2) \rightarrow Q \subset E$ by

$$
g x=\frac{f(a+\delta(a) x)}{\delta^{\prime}(f a)} .
$$

Then $g$ is $\eta$-quasisymmetric. By $6.15, g$ satisfies a Hölder condition

$$
|g(x)-g(0)| \leq C|x|^{\alpha}
$$

with $C \geq 1$ and $\alpha \leq 1$ depending only on $\varphi$ and on the numbers $d(B(1))=2$ and $d(Q)$. If $|a-b| \leq \delta(a) / 2$, then $k(a, b) \leq 1$ by $3.7(3)$. Hence $k^{\prime}(f a, f b) \leq \varphi(1)$. By $3.7(2)$, this implies $|f a-f b| \leq \delta^{\prime}(f a) e^{\varphi(1)}$, and thus $d(Q) \leq 2 e^{\varphi(1)}$. Hence $C$ and $\alpha$ depend only on $\varphi$. Since

$$
f z=\delta^{\prime}(f a) g\left(\frac{z-a}{\delta(a)}\right) \quad \text { for } z \in \bar{B}(a, \delta(a) / 2)
$$


we obtain

$$
\frac{|f a-f b|}{\delta^{\prime}(f a)}=\left|g(0)-g\left(\frac{b-a}{\delta(a)}\right)\right| \leq C\left(\frac{|b-a|}{\delta(a)}\right)^{\alpha} .
$$

12.3. Theorem. If $f: G \rightarrow G^{\prime}$ is $\varphi$-FQC, then $f$ is $\varphi_{1}$-FQC with $\varphi_{1}(t)=C\left(t^{\alpha} \vee t\right)$, where $C \geq 1$ and $0<\alpha \leq 1$ depend only on $\varphi$.

Proof. Let $D \subsetneq G$ be a domain. Let $C$ and $\alpha$ be the numbers given by 12.2. Set $t_{0}=\frac{1}{4} \wedge \varphi^{-1}(1)$. If $x, y \in D$ and $k_{D}(x, y) \leq t_{0}$, then 3.9 gives $|x-y| \leq 2 t_{0} \delta_{D}(x) \leq \delta_{D}(x) / 2$. By 12.2 and 3.9 we thus have

$$
\frac{|f x-f y|}{\delta_{f D}(f x)} \leq C\left(\frac{|x-y|}{\delta_{D}(x)}\right)^{\alpha} \leq C\left(2 k_{D}(x, y)\right)^{\alpha} .
$$

Since $k_{f D}(f x, f y) \leq \varphi\left(t_{0}\right) \leq 1$, this and 3.9 yield

$$
k_{f D}(f x, f y) \leq 2 C\left(2 k_{D}(x, y)\right)^{\alpha} .
$$

Hence we can choose $\varphi_{1}(t)=2^{1+\alpha} C t^{\alpha}$ for $0 \leq t \leq t_{0}$.

Next assume that $k_{D}(x, y)=t \geq t_{0}$. By 2.5 , we may assume that $\varphi(t) \leq M t+C_{1}$ for some $M \geq 1$ and $C_{1} \geq 0$ depending on $\varphi$. Since $f$ is $\varphi$-FQC, we have

$$
k_{f D}(f x, f y) \leq M t+C_{1} \leq\left(M+C_{1} / t_{0}\right) t .
$$

Hence we can choose $\varphi_{1}(t)=\left(M+C_{1} / t_{0}\right) t$ for $t \geq t_{0}$.

12.4. Removable sets. Suppose that $G \subset E$ is a domain and that $A$ is a subset of $G$ such that $\operatorname{int} A=\varnothing$ and such that $G \backslash A$ is a domain. We say that $A$ is removable for FQC maps if each $\varphi$-FQC map of $G \backslash A$ onto a domain in a Banach space $E^{\prime}$ can be extended to a $\varphi_{1}$-FQC map of $G$, where $\varphi_{1}$ depends only on $\varphi$. Note that the extension is unique since $\operatorname{int} A=\varnothing$. Removable sets for solid maps and for coarsely quasihyperbolic maps are defined analogously.

In $R^{n}$, all closed sets of $(n-1)$-dimensional Hausdorff measure zero are known to be removable for quasiconformal maps [Vä]. In particular, if $F \subset R^{n}$ is an affine subspace of codimension at least 2, then $F \cap G$ is removable for quasiconformal maps in any domain $G \subset R^{n}$. It is reasonable to conjecture that the corresponding result holds for FQC maps in all Banach spaces. However, only the case where $F$ is a single point, is known to be true:

12.5. TheOrem. Let $G \subset E$ be a domain and let $x_{0} \in G$. Then $\left\{x_{0}\right\}$ is removable for $\mathrm{FQC}$, solid and coarsely $\mathrm{QH}$ maps.

We omit the proof, which is rather long. The solid case is proved in detail in [II, Section 2], and the FQC case is an easy corollary. The proof for the coarsely QH case follows the same lines. To prove that the extended map is coarsely $\mathrm{QH}$, one can use Lemma 2.3 .

12.6. Notes. The Hölder continuity of $n$-dimensional quasiconformal maps was proved by F. W. Gehring $\left[\mathrm{Ge}_{1}\right.$, p. 384] in 1962. His proof was based on symmetrization and on moduli of rings, and it gives a sharp estimate for the Hölder exponent. 


\section{Open problems}

13.1. Summary. We give a collection of open questions in the free quasiworld. We recall that $E$ and $E^{\prime}$ are always real Banach spaces of dimension at least two, and that $G \subset E$ and $G^{\prime} \subset E^{\prime}$ are domains. We assume that $G \neq E$ and $G^{\prime} \neq E^{\prime}$ if this is necessary in order that the problem make sense. Remember that in the case $E=E^{\prime}=R^{n}$, a homeomorphism $f: G \rightarrow G^{\prime}$ is called $K$-quasiconformal if the metric dilatation $H(x, f)$ is bounded by $K$ in $G$.

13.2. Problems. Many of the problems can be stated for each of the four classes: QH, FQC, solid and coarsely QH maps, but we only give one version. Problems 1-12 have been mentioned earlier in this article.

1. (4.9.1) Suppose that $G \subset R^{n}, G^{\prime} \subset R^{n}$ and that $f: G \rightarrow G^{\prime}$ is $K$-quasiconformal. Is $f \varphi$-solid with $\varphi=\varphi_{K}$ ?

2. (4.9.2) Suppose that $f: G \rightarrow G^{\prime}$ is a homeomorphism such that $H(x, f) \leq K$ for all $x \in G$. Is $f \varphi$-FQC with $\varphi=\varphi_{K}$ ?

3. (5.29) Suppose that $f: G \rightarrow G^{\prime}$ is an $(M, q)$-quasisimilarity. Is $f^{-1}$ an $\left(M^{\prime}, q^{\prime}\right)$ quasisimilarity with $\left(M^{\prime}, q^{\prime}\right)$ depending only on $(M, q)$ ?

4. (6.9) Suppose that $f: G \rightarrow G^{\prime}$ is $K$-quasiconformal and that $0<q<1, B(x, r) \subset$ $G$. Is $f \mid B(x, q r) \eta$-quasisymmetric with $\eta=\eta_{K, q}$ ? In particular, is a $K$-quasiconformal map $f: R^{n} \rightarrow R^{n} \eta$-quasisymmetric with $\eta=\eta_{K}$ ?

5. (7.5) Is a fully $\varphi$-semisolid homeomorphism $f: G \rightarrow G^{\prime} \varphi_{1}$-FQC with $\varphi_{1}$ depending only on $\varphi$ ?

6. (8.3.1) Let $E$ and $E^{\prime}$ be infinite-dimensional separable Banach spaces. Is $E$ quasisymmetrically equivalent to $E^{\prime}$ ?

7. (8.3.2) Suppose that the Banach spaces $E$ and $E^{\prime}$ are quasisymmetrically equivalent. Are they bilipschitz equivalent?

8. (8.3.3) Let $1 \leq p<q<\infty$. Are the spaces $l_{p}$ and $l_{q}$ quasisymmetrically equivalent?

9. (8.16) Does there exist a solid map of a ball onto a domain with nonconnected boundary?

10. (10.23) Suppose that $G$ is a $c$-uniform domain and that the homeomorphism $f$ : $\bar{G} \rightarrow \overline{G^{\prime}}$ is $\eta$-quasimöbius rel $\partial G$ and maps $G$ onto $G^{\prime}$. Is $G^{\prime} c^{\prime}$-uniform with $c^{\prime}=c^{\prime}(c, \eta)$ ?

11. (11.18) Suppose that $f: E \rightarrow E^{\prime}$ is $\eta$-quasisymmetric. Does $f$ extend to an $\eta_{1}$-quasisymmetric map $F: E \times \mathbf{R} \rightarrow E \times \mathbf{R}$ with $\eta_{1}$ depending only on $\eta$ ?

12. (12.4) Let $F$ be a closed affine subspace of $E$ with $\operatorname{codim} F \geq 2$. Is $G \cap F$ removable for solid maps $f: G \rightarrow G^{\prime}$. In particular, is a line segment in $G$ removable for solid maps if $\operatorname{dim} E \geq 3$. For quasiconformal maps, the answer to the last question is yes by [Vä]

13. Suppose that $f: G \rightarrow G^{\prime}$ is a homeomorphism and that each point has a neighborhood $D \subset G$ such that $f_{D}: D \rightarrow f D$ is $M$-QH. Is $f M^{\prime}$-QH with $M^{\prime}=M^{\prime}(M)$ ?

14. Suppose that $G$ and $G^{\prime}$ are bounded domains with connected boundaries. Suppose also that $f: G \rightarrow G^{\prime}$ is $M$-QH and that $f$ extends to a homeomorphism $\bar{f}: \bar{G} \rightarrow \overline{G^{\prime}}$ such that $\bar{f} \mid \partial G$ is $M$-bilipschitz. Is $f M^{\prime}$-bilipschitz with $M^{\prime}=M^{\prime}(M)$ ?

In the case $E=E^{\prime}=R^{n}$, it is known that $f$ is $M^{\prime}$-bilipschitz with $M^{\prime}=M^{\prime}(M, n)$; see the proof of $\left[\mathrm{Ge}_{3}, 2.11\right]$. 
15. Suppose that $f: G \rightarrow G^{\prime}$ is a homeomorphism and that $f$ is $\eta$-quasisymmetric in the QH metric. Is $f \varphi$-FQC with $\varphi=\varphi_{\eta}$ ? The converse result is true by [I, 5.14]. In the case $E=E^{\prime}=R^{n}, f$ is clearly $K$-quasiconformal with $K=\eta(1)$.

16. Does there exist an FQC map $f: G \rightarrow G^{\prime}$ such that the cluster set $\operatorname{clus}(x, f)$ is empty for all $x \in \partial G$.

17. V. A. Zorich [Zo] proved in 1967 that if $n \geq 3$, then a local $K$-quasiconformal homeomorphism $f: R^{n} \rightarrow R^{n}$ is a homeomorphism. Does this result have an infinitedimensional version?

\section{References}

The papers $\left[\mathrm{V} \ddot{a}_{5,6,7,9}\right]$ are cited as $[\mathrm{I}],[\mathrm{II}]$, [III], [IV].

[Al] P. Alestalo, Quasisymmetry in product spaces and uniform domains, Licentiate's thesis, University of Helsinki, 1991 (Finnish).

[AG] K. Astala and F. W. Gehring, Injectivity, the BMO norm and the universal Teichmüller space, J. Analyse Math. 46 (1986), 16-57.

[Ben] Y. Benyamini, The uniform classification of Banach spaces, Longhorn notes, University of Texas, 1984-85, 15-39.

[BL] Y. Benyamini and J. Lindenstrauss, Geometric non-linear analysis, book to appear.

[Bes] C. Bessaga, Every infinite-dimensional Hilbert space is diffeomorphic with its unit sphere, Bull. Acad. Polon. Sci. Ser. Sci. Mat. Astr. Phys. 14 (1966), 27-31.

[BP] C. Bessaga and A. Pęczyński, Selected topics in infinite-dimensional topology, Polish Scientific Publishers, 1975.

[BA] A. Beurling and L. Ahlfors, The boundary correspondence under quasiconformal mappings, Acta Math. 96 (1956), 125-142.

[CC] M. Cotlar and R. Cignoli, An introduction to functional analysis, North-Holland, 1974.

[DS] D. A. DE-SPILlER, Equimorphisms and quasi-conformal mappings of the absolute, Soviet Math. Dokl. 11 (1970), 1324-1328.

[ET] V. A. EFremovich and E. S. Tihomirova, Equimorphisms of hyperbolic spaces, Izv. Akad. Nauk SSSR 28 (1964), 1139-1144 (Russian).

[Fe] H. FEDERER, Geometric measure theory, Springer-Verlag, 1969.

[Ge 1 F. W. Gehring, Rings and quasiconformal mappings in space, Trans. Amer. Math. Soc. 103 (1962), 353-393.

[Ge $\left.\mathrm{G}_{2}\right]$ F. W. GeHRIng, The Carathéodory convergence theorem for quasiconformal mappings, Ann. Acad. Sci. Fenn. Math. 336/11 (1963), 1-21.

[Ge 3 F. W. Gehring, Extension of quasiisometric embeddings of Jordan curves, Complex Variables 5 (1986), 245-263.

[GM] F. W. Gehring and O. Martio, Quasiextremal distance domains and extension of quasiconformal mappings, J. Analyse Math. 45 (1985), 181-206.

[GO] F. W. GEHRING and B. G. OsGood, Uniform domains and the quasi-hyperbolic metric, J. Analyse Math. 36 (1979), 50-74.

[GP] F. W. Gehring and B. P. PALKA, Quasiconformally homogeneous domains, J. Analyse Math. 30 (1976), 172-199. 
[GV] F. W. GEHRING and J. VÄIsÄLÄ, The coefficients of quasiconformality of domains in space, Acta Math. 114 (1965), 1-70.

[Go] S. GoŁA̧B, Quelques problèmes métriques de la géométrie de Minkowski, Prace Akademii Górniczej w Krakowie 6 (1932), 1-79 (Polish, French summary).

[GR] V. M. Goldshtein and M. Rubin, Reconstruction of domains from their groups of quasiconformal autohomeomorphisms, Preprint, 1992.

[HHM] K. HAG, P. HAG and O. MARTIO, Quasisimilarities; definitions, stability and extension, Rev. Roumaine Math. Pures Appl., to appear.

[HK] J. Heinonen and P. Koskela, Definitions of quasiconformality, Invent. Math. 120 (1995), 61-79.

[JLS] W. B. Johnson, J. Lindenstrauss and G. Schechtman, Banach spaces determined by their uniform structures, Geom. Funct. Anal. 6 (1996), 430-470.

[Jo1] P. W. Jones, Extension theorems for BMO, Indiana Univ. Math. J. 29 (1980), 41-66.

$\left[\mathrm{Jo}_{2}\right]$ P. W. Jones, Quasiconformal mappings and extendability of functions in Sobolev spaces, Acta Math. 147 (1981), 71-88.

[Kü] R. KüHNAU, Elementare Beispiele von möglichst konformen Abbildungen in der dreidimensionalen Raum, Wiss. Z. Martin-Luther-Univ. Halle-Wittenberg Math.-Natur. Reihe 11 (1962), 729-732.

[Lat] T. G. Latfullin, Criteria to the quasihyperbolicity of maps, Sibirsk. Mat. Zh. 37 (1996), 610-615 (Russian).

[Lau] D. LAugwitz, Konvexe Mittelpunktsbereiche und normierte Räume, Math. Z. 61 (1954), 235-244.

[LV] O. Lehto and K. I. Virtanen, Quasiconformal mappings in the plane, SpringerVerlag, 1973.

[Ma] O. Martio, Quasisimilarities, Rev. Roumaine Pures Appl. 36 (1991), 395-406.

[MS] O. Martio and J. Sarvas, Injectivity theorems in plane and space, Ann. Acad. Sci. Fenn. Math. 4 (1979), 383-401.

[Ri] S. Rickman, Quasiconformally equivalent curves, Duke Math. J. 36 (1969), 387-400.

$\left[\mathrm{Sc}_{1}\right]$ J. J. SCHÄFFER, Inner diameter, perimeter and girth of spheres, Math. Ann. 173 (1967), 59-82.

$\left[\mathrm{Sc}_{2}\right] \quad$ J. J. Sch ÄFFER, Geometry of spheres in normed spaces, Marcel Dekker, 1976.

$\left[\mathrm{TV}_{1}\right]$ P. TUKIA and J. VÄISÄL ̈̈, Quasisymmetric embeddings of metric spaces, Ann. Acad. Sci. Fenn. Math. 5 (1980), 97-114.

$\left[\mathrm{TV}_{2}\right]$ P. TUKIA and J. V̈̈ISÄL ̈̈, Lipschitz and quasiconformal approximation and extension, Ann. Acad. Sci. Fenn. Math. 6 (1981), 303-342.

[TV 3 P. TUKIA and J. VÄIsÄLÄ, Quasiconformal extension from dimension $n$ to $n+1$, Ann. of Math. 115 (1982), 331-348.

[Vä] J. VÄ̈s̈̈Lё, Removable sets for quasiconformal mappings, J. Math. Mech. 19 (1969), $49-51$.

[Vä2] J. V̈̈̈s̈̈LÄ, Lectures on n-dimensional quasiconformal mappings, Lecture notes in Math. 229, Springer-Verlag, 1971.

[Vä3] J. V̈̈Is⿱̈亡̈̈, Quasi-symmetric embeddings in euclidean spaces, Trans. Amer. Math. Soc. 264 (1981), 191-204.

[Vä4] J. VÄıs̈̈Lё, Quasimöbius maps, J. Analyse Math. 44 (1984/85), 218-234.

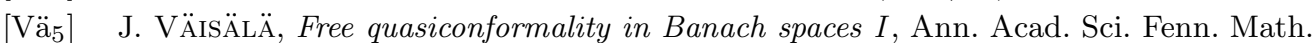
15 (1990), 355-379. 
[Vä6] J. VÄıs̈̈LÄ, Free quasiconformality in Banach Spaces II, Ann. Acad. Sci. Fenn. Math. 16 (1991), 255-310.

[Vä7] J. VÄıs̈̈Lё, Free quasiconformality in Banach spaces III, Ann. Acad. Sci. Fenn. Math. 17 (1992), 393-408.

[Vä8] J. VÄı̈̈̈̈ё, Banach spaces and bilipschitz maps, Studia Math. 103 (1992), 291-294.

[Vä9] J. VÄ̈s⿱̈亡̈̈̈, Free quasiconformality in Banach spaces IV, Analysis and Topology, ed. by C. Andreian Cazacu et al., World Scientific, 1998.

[Vu] M. Vuorinen, Conformal invariants and quasiregular mappings, J. Analyse Math. 45 (1985), 69-115.

[Zo] V. A. Zorich, The theorem of M. A. Lavrent'ev on quasiconformal mappings, Math. USSR - Sbornik 3 (1967), 389-403.

\section{Index}

broken tube 96

cigar condition 101

cigar theorem 105

cigar 101

cluster set 108

coarse cigar theorem 106

coarse length 99

coarsely bilipschitz map 59

coarsely Lipschitz map 59

coarsely quasiconvex space 99

coarsely quasihyperbolic map 65

cross ratio 79

diameter cigar 101

differentiable map 70

$\mathrm{FQC}=$ freely quasiconformal 65

free quasiworld 57

freely quasiconformal map 65

fullness 65

fundamental lemma 109

half space 92

Hölder condition 78, 113

inversion 68

length cigar 101

Lipschitz map 59

locally bilipschitz map 69

locally quasisymmetric map 76, 86

logarithmic distance 63

metric definition of quasiconformality 56

metric dilatation 56

neargeodesic 97

organ pipe domain 94

$\mathrm{QH}=$ quasihyperbolic 61

quantitativeness 59 quasiconformal, freely 65

quasiconvex space 59

quasihyperbolic length 61

quasihyperbolic map 65

quasihyperbolic metric 61

quasihyperbolically uniform domain 101

quasimöbius map 79

quasisimilarity 71

quasisymmetric map 73

radial bilipschitz map 71

radial power map 71

reflection 112

relative distance 63

relative map 84

relative quasimöbius 80

relative quasisymmetry 78

removable set 114

ring property 86

semisolid map 84

solid arc theorem 100

solid arc 100

solid map 65

sperical ring 90

stretching 67

tower 93

tube 95

turning condition 101

uniform domain 101

uniformity conditions 101

uniformly continuous map 58

weakly locally quasisymmetric map 86

weakly quasisymmetric map 74

whole space 90 